\title{
Intelligent Front-end Sample Preparation Tool using Acoustic Streaming
}

Darren W. Branch, Erika J. Cooley, Gennifer Smith, Thayne L. Edwards, Conrad D. James, Jamie McClain, Jaclyn Murton, John M. Anderson, Joe Kotulski, and Paul G. Clem

Prepared by

Sandia National Laboratories

Albuquerque, New Mexico 87185 and Livermore, California 94550

Sandia is a multiprogram laboratory operated by Sandia Corporation,

a Lockheed Martin Company, for the United States Department of Energy's

National Nuclear Security Administration under Contract DE-AC04-94AL85000.

Approved for public release; further dissemination unlimited. 
Issued by Sandia National Laboratories, operated for the United States Department of Energy by Sandia Corporation.

NOTICE: This report was prepared as an account of work sponsored by an agency of the United States Government. Neither the United States Government, nor any agency thereof, nor any of their employees, nor any of their contractors, subcontractors, or their employees, make any warranty, express or implied, or assume any legal liability or responsibility for the accuracy, completeness, or usefulness of any information, apparatus, product, or process disclosed, or represent that its use would not infringe privately owned rights. Reference herein to any specific commercial product, process, or service by trade name, trademark, manufacturer, or otherwise, does not necessarily constitute or imply its endorsement, recommendation, or favoring by the United States Government, any agency thereof, or any of their contractors or subcontractors. The views and opinions expressed herein do not necessarily state or reflect those of the United States Government, any agency thereof, or any of their contractors.

Printed in the United States of America. This report has been reproduced directly from the best available copy.

Available to DOE and DOE contractors from

U.S. Department of Energy

Office of Scientific and Technical Information

P.O. Box 62

Oak Ridge, TN 37831

Telephone: $\quad$ (865)576-8401

Facsimile: $\quad$ (865)576-5728

E-Mail: reports@adonis.osti.gov

Online ordering: http://www.osti.gov/bridge

Available to the public from

U.S. Department of Commerce

National Technical Information Service

5285 Port Royal Rd

Springfield, VA 22161

Telephone: $\quad$ (800)553-6847

Facsimile: $\quad$ (703)605-6900

E-Mail: orders@ntis.fedworld.gov

Online order: http://www.ntis.gov/help/ordermethods.asp?loc=7-4-0\#online

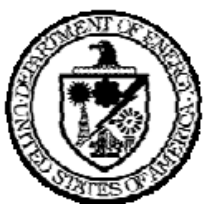


SAND2009-6193

Unlimited Release

Printed September 2009

\title{
Intelligent Front-end Sample Preparation Tool using Acoustic Streaming
}

Darren W. Branch, Erika J. Cooley, Gennifer Smith, Thayne L. Edwards, Conrad D. James, Komandoor Achyuthan, Jamie McClain, Jaclyn Murton, John M. Anderson, Joe Kotulski, and Paul G. Clem

Biosensors and Nanomaterials Department

Sandia National Laboratories

PO Box 5800

Albuquerque, NM 87185-1425

\begin{abstract}
We have successfully developed a nucleic acid extraction system based on a microacoustic lysis array coupled to an integrated nucleic acid extraction system all on a single cartridge. The microacoustic lysing array is based on 36 o Y cut lithium niobate, which couples bulk acoustic waves (BAW) into the microchannels. The microchannels were fabricated using Mylar laminates and fused silica to form acoustic-fluidic interface cartridges. The transducer array consists of four active elements directed for cell lysis and one optional BAW element for mixing on the cartridge. The lysis system was modeled using one dimensional (1D) transmission line and two dimensional (2D) FEM models. For input powers required to lyse cells, the flow rate dictated the temperature change across the lysing region. From the computational models, a flow rate of 10

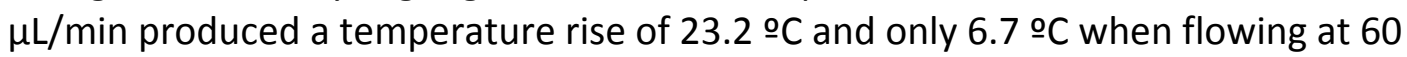
$\mu \mathrm{L} / \mathrm{min}$. The measured temperature changes were 5 o $\mathrm{C}$ less than the model. The computational models also permitted optimization of the acoustic coupling to the microchannel region and revealed the potential impact of thermal effects if not controlled. Using $E$. coli, we achieved a lysing efficacy of $49.9 \pm 29.92 \%$ based on a cell viability assay with a $757.2 \%$ increase in ATP release within 20 seconds of acoustic exposure. A bench-top lysing system required 15-20 minutes operating up to 58 Watts to achieve the same level of cell lysis. We demonstrate that active mixing on the cartridge was critical to maximize binding and release of nucleic acid to the magnetic beads. Using a sol-gel silica bead matrix filled microchannel the extraction efficacy was $40 \%$. The cartridge based magnetic bead system had an extraction efficiency of $19.2 \%$. For an electric field based method that used Nafion films, a nucleic acid extraction
\end{abstract}


efficiency of $66.3 \%$ was achieved at 6 volts DC. For the flow rates we tested (10 -50 $\mu \mathrm{L} / \mathrm{min})$, the nucleic acid extraction time was 5-10 minutes for a volume of $50 \mu \mathrm{L}$. Moreover, a unique feature of this technology is the ability to replace the cartridges for subsequent nucleic acid extractions. 


\section{TABLE OF CONTENTS}

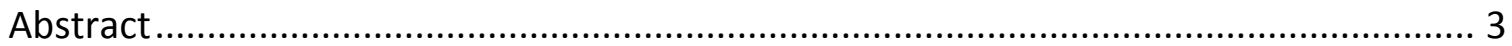

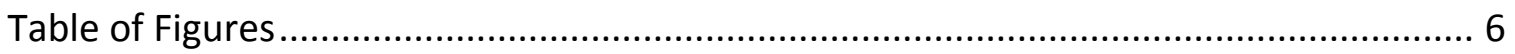

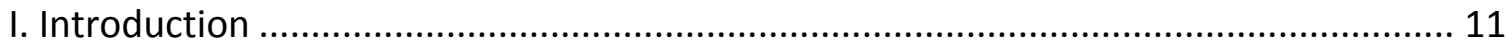

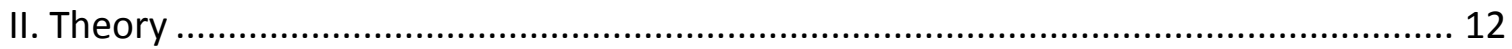

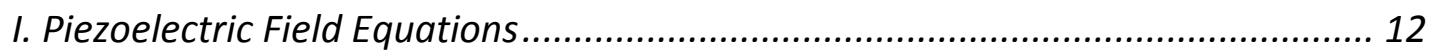

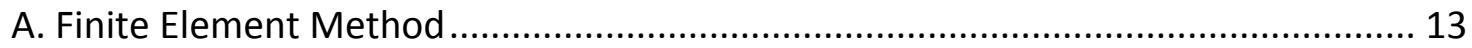

I. FEM Extraction of Electrical Impedance and Electromechanical Coupling............ 14

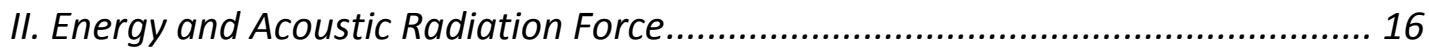

III. Thermal Analysis of Acoustic Transducer Array................................................. 16

B. Transmission Line Method................................................................................ 18

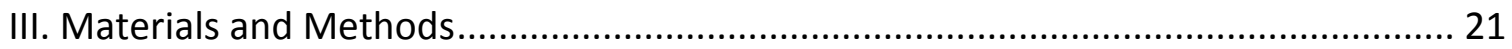

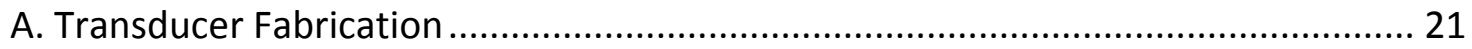

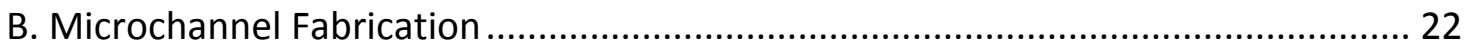

I. PDMS Microfluidic Channel............................................................................. 22

II. DNA Binding Paramagnetic Beads Based Cartridge ........................................ 22

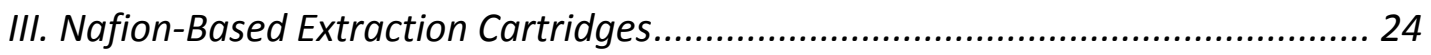

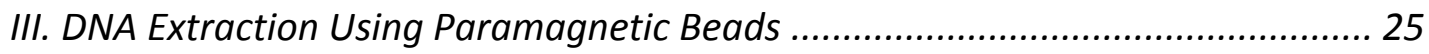

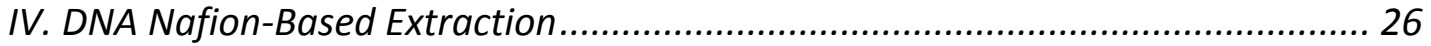

C. Nucleic Acid Extraction Approaches .................................................................... 27

D. Cell Culture .................................................................................................... 28

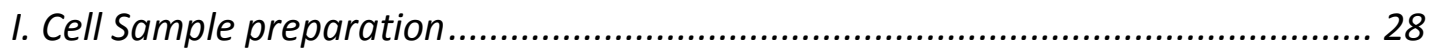

II. Sample Sonication using a Commercial System ............................................... 28

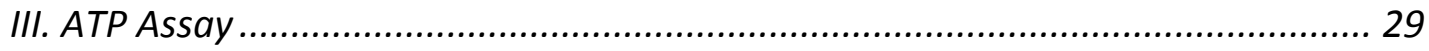

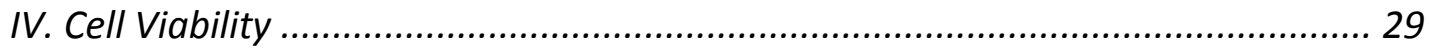

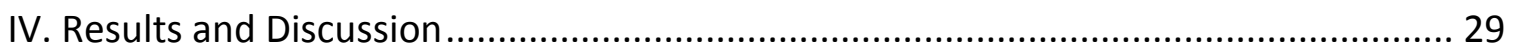

A. Transmission Line Model of Bulk Acoustic Wave Transducer Array ....................... 29

B. FEM Analysis of Bulk Acoustic Wave Transducer Array ........................................ 32

I. Impedance of Transducer and Composite Structure............................................ 32

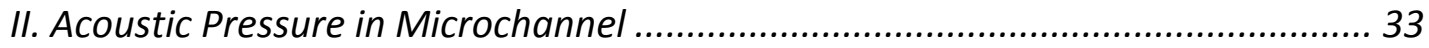

III. Energy from Acoustic Fields in Microchannel ................................................... 34

V. Thermal Analysis.......................................................................................... 35

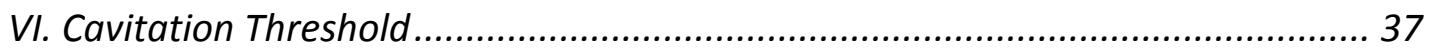

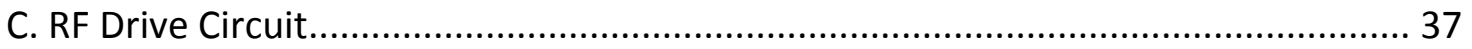

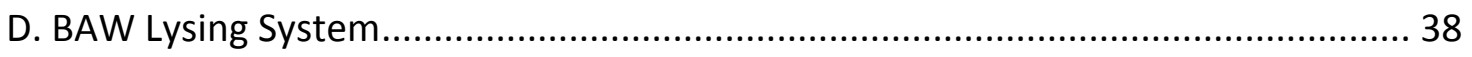

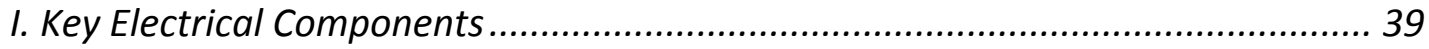

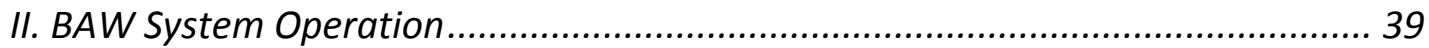

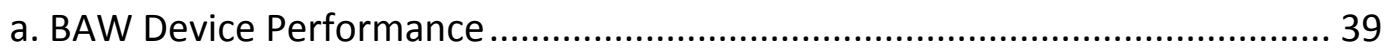

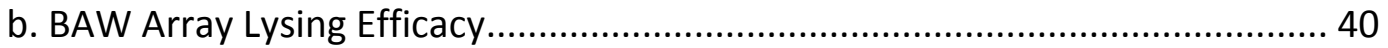

E. Nucleic Acid Extraction ......................................................................................... 43

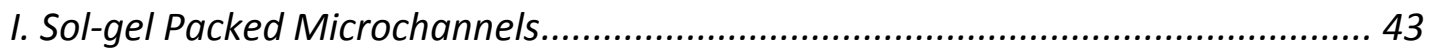

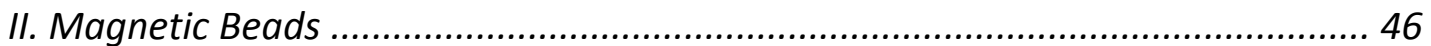

III. Nafion Coated Electrodes............................................................................. 48

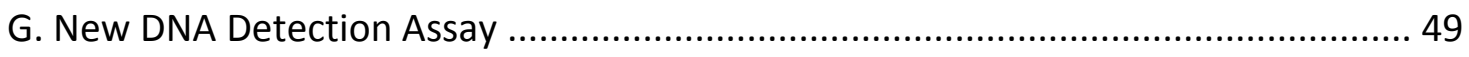


.

III. Cyanine 1 optimization for detecting nucleic acids ........................................ 52

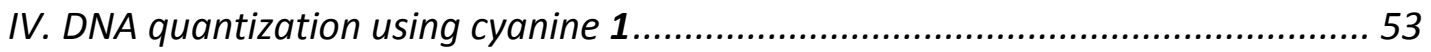

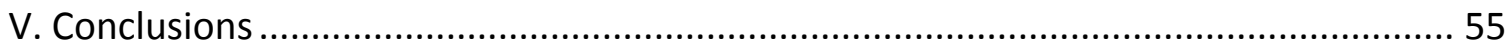

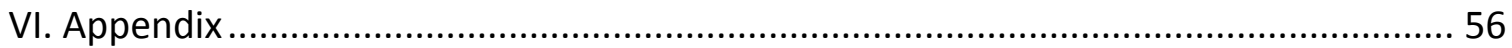

VII. Developed Computer Codes ........................................................................... 56

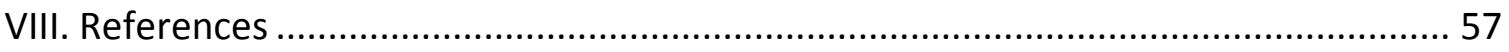

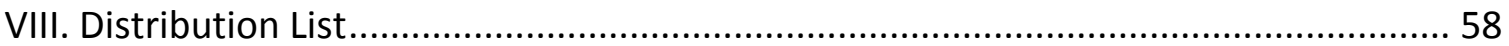

\section{Table of Figures}

Figure 1 Geometry of the acoustic lysing array. a) The acoustic transducers were modeled as a heat flux discontinuities where the surface of the transducer in contact with the fused silica was given an inward heat flux $Q_{0}$. b) Depicts the left hand side of the modeled geometry where the laminar flow condition was established [Eq.(1.20)]. The fluid input (red region) which was modeled as a fully developed laminar condition. c) The detailed view shows the position of the fluidic channel aligned along the central region of the acoustic transducers.

Figure 2 Physical parameters of the nth piezoelectric layer of the transducer which is represented by a three port circuit. The 1-D model assumes that the total thickness of the multilayer transducer is $<10 \%$ of any lateral dimension. The velocity, force, potential, and current at the acoustic ports are $v_{n}, F_{n}, U_{n}$, and $I_{n}$. $h_{n}$ is the polarization of the material of layer $n$. 18

Figure 3 BAW transducer array. The first four transducer elements are used for cell lysis and the final element is for mixing solutions in the microchannel. This transducer array couples to the microchannels through a fused silica boundary, where remote actuation of the fluid is accomplished by the propagation of acoustic waves. Each transducer is $3 \mathrm{~mm}$ in diameter. The transducer elements shown in brown are addressed by $\mathrm{Au} / \mathrm{Cr}$ electrodes patterned using a shadow mask process.

Figure 4 Scale drawing of BAW transducer array assembly. Assembly process begins with the application of the transducer Mylar mask to the fused silica substrate. The BAW transducers are bonded to the Au electrodes using Epotek 4110 epoxy. By masking the backside of the transducers, the region defined as the confinement mask is backfilled with Epotek 301 epoxy. 22

Figure 5 Lysing and nucleic acid extraction microchannels based on paramagnetic beads, a) Cells are lysed in the region defined in Layer $\# 2$ and then the nucleic acid-bead complex is trapped by a magnetic field. The two magnetic regions were 0.25 " in diameter. b) Lysing, extraction, and mixing cartridge. This design added acoustic mixing to release DNA from the magnetic beads in the presence of the elution buffer. The magnetic trap was reduced to $0.125^{\prime \prime}$ in diameter. A final magnetic trap was used to prevent bead loss from the microchannel cartridge. Both devices were $4.98 \mathrm{~mm}$ thick. 
Composite device was $4.95 \mathrm{~mm}$ thick. AMA: adhesive /Mylar /adhesive, FS: Fused silica, MA: Mylar /adhesive, PMMA: Polymethylmethacrylate. Both designs were $15.3 \mathrm{~mm}$ x $39.6 \mathrm{~mm}$.

Figure 6 BAW lysis and Nafion based nucleic extraction cartridge. a) This design used Nafion coated Au on Mylar to trap DNA after release from the cells. The Nafion film prevented the DNA from reaching the surface of the electrodes and hence retained its biological activity. Composite device was $4.95 \mathrm{~mm}$ thick. AMA: adhesive /Mylar /adhesive, FS: Fused silica, MA: Mylar /adhesive, PMMA: Polymethylmethacrylate. Both designs were $15.3 \mathrm{~mm} \times 39.6 \mathrm{~mm}$. 25 Figure 7 Nucleic acid extraction methods for a complete single cartridge solution. a) Solgel silica bead matrix based extraction. The silica matrix was fabricated by injecting or patterning sol-gel matrix containing silica beads, b) Magnetic bead based nucleic acid extraction. Nucleic acid binding magnetic beads $\left(10^{6}\right.$ beads $\left./ \mathrm{ml}\right)$ were mixed with raw cellular samples prior to lysis. A pH shift from 6.5 to 8.5 eluted the purified nucleic acid, c) Nafion coated electrode based extraction. A Nafion film was applied to gold electrodes on a Mylar substrate to electrostatically bind and release nucleic acid. Figure 8 Cross-section of BAW transducer and composite structure. a) Unloaded transducer. b) Composite model of the transducer which shows the required layers to permit removal of the microchannel cartridges from the BAW transducer. For the impedance calculations $V=1 \mathrm{~V}$.

Figure 9 Impedance response of 36ㅇ $Y$ lithium niobate transducer using the 1D Transmission line method. Though the unloaded transducer exhibits excellent resonance near $55 \mathrm{MHz}$, the impedance was far from optimal $\mathrm{Z}(55 \mathrm{MHz})=1.10-\mathrm{j} 7.15 \Omega$. For the composite transducer, $\mathrm{Z}(55 \mathrm{MHz})=44.67-\mathrm{j} 16.9 \Omega$, which a much better match to a $50 \mathrm{Ohm}$ system. Perfect matching was undesirable since this would require exquisite tracking of the maximal drive frequency. Therefore, broadband behavior was required for this application.

Figure 10 Return Loss (S11) for the unloaded and composite transducers based on 1D transmission line models. After proper layer-to-layer matching the return loss at the resonance frequency provided an excellent match to a $50 \mathrm{ohm}$ source. The return loss for the composite transducer was $-14.5 \mathrm{~dB}$ with a tunable range of $8 \mathrm{MHz}$ based on maintaining $90 \%$ input power coupling to the transducers.

Figure 11 Extracted impedance response of 36ㅇ Y lithium niobate transducer from 2D FEM. The calculation was based on 2D FEM of the transducer with infinitesimally thin Au electrodes in air.

Figure 12 Acoustic pressure within the microchannel cross-section. a) Acoustic pressure at $54 \mathrm{MHz}$. The acoustic pressure was $\sim 1 \mathrm{MPa}$ for an input of $3 \mathrm{~V} \mathrm{P}-\mathrm{P}, \mathrm{b})$ Acoustic pressure at several drive frequencies across the width of the microchannel, c) Acoustic pressure at several drive frequencies across the height of the microchannel. 34 Figure 13 Kinetic and potential energy of the acoustic fields in the center of microchannel. 35

Figure 14 3D Thermal model of BAW transducer array. This analysis was done at $u_{\max }=$ $0.02 \mathrm{~m} / \mathrm{s}$ which is $60 \mu \mathrm{l} / \mathrm{min}$. The channel inlet (lower left) starts at $300 \mathrm{~K}$, reaching a 
steady state value of $306 \mathrm{~K}$ at the outlet (upper right). The input power to each transducer was $21.9 \mathrm{dBm}$.

Figure 15 Steady state thermal response versus the input power to the BAW lysing transducer. The open blue circles are the experimentally measured temperature near the microchannel outlet.

Figure 16 Schematic of RF drive circuit for BAW lysing system. Computer control was used to tune the frequency of the RF source (output power $-0 \mathrm{dBm}$ ) which was then connected to a $2 \mathrm{~W}$ RF power amplifier. This was split 4 ways to drive each BAW transducer.

Figure 17 BAW lysing components. a) Nucleic acid binding magnetic bead based cartridge, b). Nafion coated gold electrodes reversibly bind nucleic acid and c). Complete lysing/nucleic acid extraction system.

Figure 18 Comparison of cell lysis efficacy for commercial and microacoustic methods. a) Cell culture plating viability assay, b) ATP assay to determine cell lysis. 'Glass' and 'Plastic' refer to the coupling layer between the transducer and microchannel. It was expected that glass would be less acoustically lossy than plastic and hence improve cell lysis. The data for the two microfluidic systems were measured at $10 \mu \mathrm{L} / \mathrm{min}$ flow rates. $\mathrm{N}=10$.

Figure 19 Cell lysis efficacy the BAW transducer array versus input power, a) Glass base, b) Plastic base. The flow rate was $10 \mu \mathrm{L} / \mathrm{min} . \mathrm{N}=10$.

Figure 20 Sol-gel silica bead matrix extraction cartridge. a) Prototype DNA extraction microchamber. Chip dimensions were $45 \times 15 \times 1.25 \mathrm{~mm}$. b) Injection and spray methods for integrating the DNA extraction matrix into the microchamber. 44

Figure 21 Sol-gel-bead matrix cured within a closed microchamber.

Figure 22 Patterning of DNA extraction matrix (top). After removal of patterning mask (bottom).

Figure 23 DNA extraction using a silica-bead matrix. a) Packed microchannel, b) Actual DNA extraction device, c) End of the packed bed section of sol-gel immobilized silica beads $(5 \mu \mathrm{m}$ ) in channel, d) Extraction profile (black) and cumulative extraction (red) of DNA from a packed bed device as a function of collected fraction. The extraction efficiency was $40 \%$. 46

Figure 24 Mass of DNA extracted using ChargeSwitch ${ }^{\circledR}$ beads with microfluidic cartridge designs 1 and 2 and a sample flow rate of $10 \mu \mathrm{L} / \mathrm{min}$. 47 Figure 25 Mass of DNA extracted using ChargeSwitch ${ }^{\circledR}$ beads with microfluidic cartridge design 3 and a sample flow rate of $50 \mu \mathrm{L} / \mathrm{min}$. 48 Figure 26 DNA extraction from Nafion coated electrodes. Increasing the applied voltage dramatically improved DNA binding. The injected amount of DNA was $90 \mathrm{ng} . . . \ldots \ldots . . . . . .49$ Figure 27 Molecular structures of the cyanines....................................................... 50 Figure 28 Effects of methanol concentration on the self-assembly of cyanine 1 . The tracing with open circles is $\phi X 174$ DNA, closed circles $\lambda D N A$, open squares Escherichia coli genomic DNA and closed squares Saccharomyces cerevisiae RNA.

Figure 29 Effects of salt concentration on the self-assembly of cyanine 1 . The tracing with open circles is $\phi X 174$ DNA, closed circles $\lambda$ DNA, open squares Escherichia coli genomic DNA and closed squares Saccharomyces cerevisiae RNA. 
Figure 30 Optimization of cyanine 1 concentration for the detection and quantization of фX174 DNA. Increasing concentrations of 1 were mixed with 450 pM $\phi X 174$ virion DNA in a total volume of $100 \mu \mathrm{L}$ ( $45 \mathrm{fmol} D N A$ ) and fluorescence intensity was measured by exciting the samples at $425 \mathrm{~nm}$ and emission at 470 $\mathrm{nm}$.

Figure 31 Quantization of $\phi X 174$ DNA using the supramolecular self-assembly of cyanine 1. Increasing amounts of $\phi X 174$ DNA were reacted with $5 \mu \mathrm{M} 1$. The closed circles data were collected with $355 \mathrm{~nm}$ (ex) and $460 \mathrm{~nm}$ (em) filters (left, Y-1 axis). The open

diamonds data were obtained with $400 \mathrm{~nm}$ (ex) and $460 \mathrm{~nm}$ (em) filters (right, Y-2 axis). The cross symbols data were obtained using the monochromator-based instrument with $426 \mathrm{~nm}(e x)$ and $470 \mathrm{~nm}$ (em) (left, Y-1 axis). Linearity $\left(r^{2}\right)$ of the dose-response curves were: open diamonds, 0.99192; closed circles, 0.99830; crosses, $0.99675 \ldots \ldots \ldots \ldots \ldots . . . . . . . .54$ 


\section{Introduction}

The need to rapidly extract and process genomic material for identification of biological agents and disease is an ever increasing requirement. Methods that allow the complete unbiased isolation of nucleic acids from microorganisms and cellular samples are necessary to aid in molecular analysis methods and detection schemes. The biases implicit in samples makes it essential to develop preparation methods that directly access the nucleic acid content for field portable systems. This requires overcoming a variety of interferences that diminish quality, yield, and diversity of extracted nucleic acids. Routine laboratory methods for cell lysis include freeze/thaw, proteinase $K$, lysozyme, and guanidium salt treatments, ethanol or 2-propanol precipitation, DNA; ballistic disintegration; or sonication at low frequencies (e.g. kilohertz) after pretreatment with other chemicals [1]. A key aspect is that the extraction format must be highly scalable to benefit many types of biodetection systems already in place.

Many of the current nucleic acid extraction techniques, however, require significant manual intervention and consumables leading to limitations that are especially relevant for the unattended, timely detection of biological warfare agents or other microorganisms in a complex milieu. The continued reliance on large laboratory equipment (e.g. centrifuges, gel electrophoresis units, ultracentrifuges) and most require chemical or enzymes that are labile or need special handling, storage and disposal further impede progress toward miniaturized autonomous detection. Ultrasonic waves are known to induce significant pressure variation [2] and induce cavitation within fluids. Thus, acoustic waves prove to be an ideal lysing mechanism which is compatible with sealed microsystems. Acoustic methods avoid the use of harsh chemicals which often interfere with subsequent detection methods (e.g. PCR). Often the altered $\mathrm{pH}$ and chemical background adds additional steps that can otherwise be avoided. Recently, large-scale acoustic transducers have proven powerful for disrupting cell membranes and spores for subsequent DNA analysis [1, 3]. Thin-film based ultrasonic actuators have also been used lyse cellular samples and have proven more effective for microsystem applications [4, 5]. Thin film based actuators suffer from limitations in attainable film thickness and also meeting the thermal requirements for long-term use. The deposition thicknesses that can be reasonably attained are between 1 to $10 \mu \mathrm{m}$, which translates to range of $316 \mathrm{MHz}$ to $3.165 \mathrm{GHz}$ for $\mathrm{ZnO}$. Even the lowest frequency of $316 \mathrm{MHz}$ creates fabrication difficulties and also reduces the coupling to the fluid. A frequency between 100 and $300 \mathrm{MHz}$ is far more optimal for coupling into the fluid. Moreover, these devices must be strictly operated in a pulsed mode to prevent device damage.

A second major problem is that nearly all microsonicator approaches lack on-chip nucleic acid extraction processing capability. Thus, the lysate must be processed and purified off-chip, reducing the effectiveness of a microsystem solution. Nucleic acid purification methods that are suitable for on-chip use include using silicon based microstructures [6], commercial nucleic acid binding media [7], silica beads in the presence of chaotropic salts [8], and silica matrices [9]. Though packed silica beads bind 
and elute nucleic acids, their inherent instability due to compression causes widely varying results. This limitation has been overcome by using a gelled sol-gel solution of silica beads to stabilize the matrix, improving reproducibility [9]. Recently another powerful nucleic acid extraction methods has been demonstrated which uses Nafion coated gold films to reversibly capture nucleic acids [10]. This method is particularly easy to implement in microfluidic format and only needs low DC voltages for operation. To aid in analysis and device optimization, 1-D transmission line and finite element method (FEM) were used to calculate the acoustic pressure and velocity fields in the fluid filled microchannels. Using these computed fields the kinetic, and potential energy were calculated. This permitted determination of the force profile within the microchannels. The acoustic actuators were designed to create large pressure fields ( $10 \mathrm{MPa}$ ) in microfluidic channels to achieve efficient lysing activity. It was crucial that the lysing mechanism occurred due to the acoustic pressure fields alone, rather than from localized heating during acoustic excitation. For this reason, additional layers and thermal management were needed for impedance matching while mitigating any heat generated by the actuators.

In this work, we present the development of an acoustic based nucleic acid extraction system. Using a bulk acoustic wave (BAW) transducer array, acoustic waves are coupled into microfluidic cartridges. DNA was extracted using three approaches, silica based solgel filled microchannels, nucleic binding magnetic beads, and Nafion coated gold electrodes. By using the term nucleic acid, we refer to the DNA or RNA content present in the lysate, though the expected RNA extraction is minimal since no additional inhibitors were introduced. The subsequent experimental analysis only detected the amount of extracted DNA.

\section{Theory}

\section{Piezoelectric Field Equations}

Acoustic waves must satisfy both Newton's and Maxwell's equations. In the absence of external forces, the equations are expressed as

$$
\begin{gathered}
\rho \frac{\partial^{2} u_{i}}{\partial t^{2}}=\nabla \cdot T \\
S=\nabla_{s} u \\
\nabla \cdot D=\rho_{f}
\end{gathered}
$$

where $\rho$ is the mass density, $u$ is the particle displacement, and $T$ and $S$ are the surface stress and strain components, respectively. $D$ and $\rho_{f}$ are the electric displacement and free charge density, respectively. The free charge density $\rho_{f}$ is zero everywhere except at the surface of the substrate.

In a piezoelectric substrate, the coupled constitutive equations for piezoelectric media are given by: 


$$
\begin{gathered}
T_{i j}=c_{i j k l}^{E} S_{k l}-e_{k i j}^{t} E_{k} \\
D_{i}=e_{i k l} S_{k l}+\varepsilon_{i k}^{S} E_{k}
\end{gathered}
$$

where $e$ and $c^{E}$ are the piezoelectric stress constants and stiffness constants. Since the coupling between the electric and elastic fields is weak, the magnetic fields can be neglected and the electric fields derived from the scalar potential. This is known as the static field approximation in which the particle displacements $u_{i}$ are along the coordinate axis $x_{i} . \ln (0.4)$ and (0.5), we recognize Hooke's law and $D=\varepsilon E$, where $E=-\nabla \phi$ and $\phi$ is the electrical potential on the surface. By substituting (0.4) and (0.5) into $(0.1)$ and $(0.3)$ yields,

$$
\begin{gathered}
\rho \frac{\partial^{2} u}{\partial t^{2}}=\nabla \cdot c^{E}: \nabla_{s} u-\nabla \cdot(e \cdot E) \\
\nabla \cdot\left(e: \nabla_{s} u\right)-\nabla \cdot\left(\varepsilon^{s} \cdot \nabla \phi\right)=0
\end{gathered}
$$

\section{A. Finite Element Method}

In the finite element modeling (FEM) formalism for piezoelectricity the partial differential equations that relate the stresses to the particle displacement and electric displacement to the free volume charge density are replaced with finite number of unknowns [11] and used to solve (0.4) and (0.5). The piezoelectric transducer was analyzed using Comsol Multiphysics (Comsol, Los Angeles, CA), a Multiphysics FEM tool. A triangular mesh was used for the entire structure.

In the fluid region the acoustic waves are governed by the frequency-domain Helmholtz equation for determining the acoustic pressure,

$$
\nabla \cdot\left(-\frac{1}{\rho_{f}}(\nabla p-q)\right)-\left(\frac{\omega^{2}}{\rho_{f} c_{f}^{2}}\right) p=Q
$$

where $p$ is the acoustic pressure $(\mathrm{Pa}), \rho_{f}$ is the fluid density $\left(\mathrm{kg} / \mathrm{m}^{3}\right), c_{f}$ is the complex acoustic velocity $(\mathrm{m} / \mathrm{s})$ in the medium, $\omega$ is the angular frequency $(\mathrm{rad} / \mathrm{s}), q$ is a dipole source term $\left(\mathrm{N} / \mathrm{m}^{3}\right)$, and $Q$ is the monopole source term $\left(1 / \mathrm{s}^{2}\right)$. The inclusion of acoustic loss in the fluid region is introduced by allowing the fluid density $\left(\rho_{f}\right)$ and acoustic speed $\left(c_{f}\right)$ to be complex quantities,

$$
\rho_{f}=\frac{Z_{f} k_{f}}{\omega} \quad c_{f}=\frac{\omega}{k_{f}} \quad k_{f}=\frac{\omega}{c_{s}}-i \alpha \quad Z_{f}=\rho_{o} c_{s}
$$

where $Z_{f}$ is the complex acoustic impedance $\left(\mathrm{Pa} \cdot \mathrm{s} / \mathrm{m}^{2}\right), \alpha$ is the attenuation coefficient $(1 / \mathrm{m})$. In the absence of damping (i.e. $\alpha=0), \rho_{f}=\rho_{o}$ and $c_{f}=c_{s}$.

The piezoelectric transducer was excited by application of $V_{o}=1 \mathrm{~V}$ to the top edge while the bottom surface had $V=0$, where the electrodes were modeled as infinitely thin.

The edges of the transducer were fixed with the electrode surfaces allowed to move 
freely to approximate a real transducer. This boundary required that the displacements $u_{x}$ and $u_{y}=0$. The top and bottom surface of the transducer were free to move.

Continuity of stresses and displacements were imposed on the internal boundaries of the aluminum heat sink, acrylic, piezoelectric transducer and fused silica. The motion of the solid regions produces normal acceleration $(a)$ at the interfaces between the fluid and acrylic layer given as

$$
a_{x} \cdot n_{x}+a_{y} \cdot n_{y}=n \cdot\left(\frac{1}{\rho_{c}}(\nabla p)\right)
$$

This equation couples the motion of the transducer, aluminum, and fused silica layers into acceleration of the fluid. In turn the fluid pressure produces a load on the acrylic and fused silica regions. This load was included since the fluid pressure is not negligible as in the case of an air domain in contact with the silicon. Fluid loads the acrylic and fused silica layers as

$$
\begin{aligned}
& F_{x}=-p \cdot n_{x} \\
& F_{y}=-p \cdot n_{y}
\end{aligned}
$$

where $p$ is the pressure in the fluid $(\mathrm{Pa})$, and $n_{x}$ and $n_{y}$ are the normal components at the fluid-structure interfaces.

\section{FEM Extraction of Electrical Impedance and Electromechanical Coupling}

In the 2D FEM analysis the impedance was computed using

$$
Z(\omega)=\frac{V}{\sum_{i} n \cdot J_{i}(\omega)}
$$

where $V$ was the amplitude of the driving voltage $(V)$, and $n \cdot J$ is the current density outflow $\left(\mathrm{A} / \mathrm{m}^{2}\right)$ at the nodes along the driven electrode boundary. The current density was computed on the driven electrode at each frequency. For comparison with measurement the impedance was converted to return loss (S11),

$$
S 11(\omega)=\frac{Z(\omega)-Z_{o}}{Z(\omega)+Z_{o}}
$$

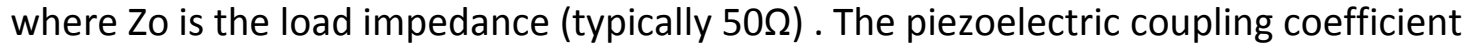
was calculated from the material parameters as

$$
K_{33}=\sqrt{\frac{e_{33}^{2}}{\varepsilon_{0} \varepsilon_{r}^{S} c_{33}^{E}+e_{33}^{2}}}
$$

where $e_{33}$ is the piezoelectric coefficient $\left(\mathrm{C} / \mathrm{m}^{2}\right), \varepsilon_{0}$ is the permittivity of free space, $\varepsilon_{r}^{S}$ is the relative permittivity at constant strain, and $c_{33}^{E}$ is the stiffness at constant electric field. (Note: this piezoelectric coupling coefficient differs from $k$ which is defined as $\kappa=\sqrt{e_{33}^{2} / \varepsilon^{S} c^{E}}$ with $\left.c^{E}=c^{D}-e^{2} / \varepsilon^{S}\right)$. 
From the FEM results the piezoelectric coupling was computed as

$$
K_{33(F E M)}=\sqrt{\frac{\pi}{2} \frac{f_{r}}{f_{a}} \tan \left[\left(\frac{f_{r}-f_{a}}{f_{a}}\right) \frac{\pi}{2}\right]}
$$

where $f_{r}$ is the resonant frequency, $f_{a}$ is the anti-resonant frequency of the transducer. The material constants for 36 o Y cut lithium niobate (Boston Piezo-Optics, Inc, Bellingham, MA) are shown in Table 1. For propagation along the $z$-axis (Fig. $x$ ), the longitudinal velocity was computed from the material constants in Table 1 as $v=7340$ $\mathrm{m} / \mathrm{s}$ for the thickness extension mode. For fused silica, the material constants are $E=70$ $\cdot 10^{9} \mathrm{~N} / \mathrm{m}^{2}, v=0.17$, and $\rho=2200 \mathrm{~kg} / \mathrm{m}^{3}$. The material constants for acrylic are $E=9$. $10^{9} \mathrm{~N} / \mathrm{m}^{2}, u=0.37, \rho=1190 \mathrm{~kg} / \mathrm{m}^{3}, \alpha=6.4 \mathrm{~dB} / \mathrm{cm} @ 5 \mathrm{MHz}$. For Mylar, the material constants are $E=7.6 \cdot 10^{9} \mathrm{~N} / \mathrm{m}^{2}, v=0.37$, and $\rho=1190 \mathrm{~kg} / \mathrm{m}^{3}$. The water was modeled as an ideal fluid with $\rho_{f}=1000 \mathrm{~kg} / \mathrm{m}^{3}$ and $c_{f}=1500 \mathrm{~m} / \mathrm{s}$. The material constants for the E. coli were $\rho_{p}=1050 \mathrm{~kg} / \mathrm{m}^{3}, c_{p}=1700 \mathrm{~m} / \mathrm{s}$, and $r_{p}=1 \mu \mathrm{m}$.

Table 1 Acoustic Properties of Transducer Materials

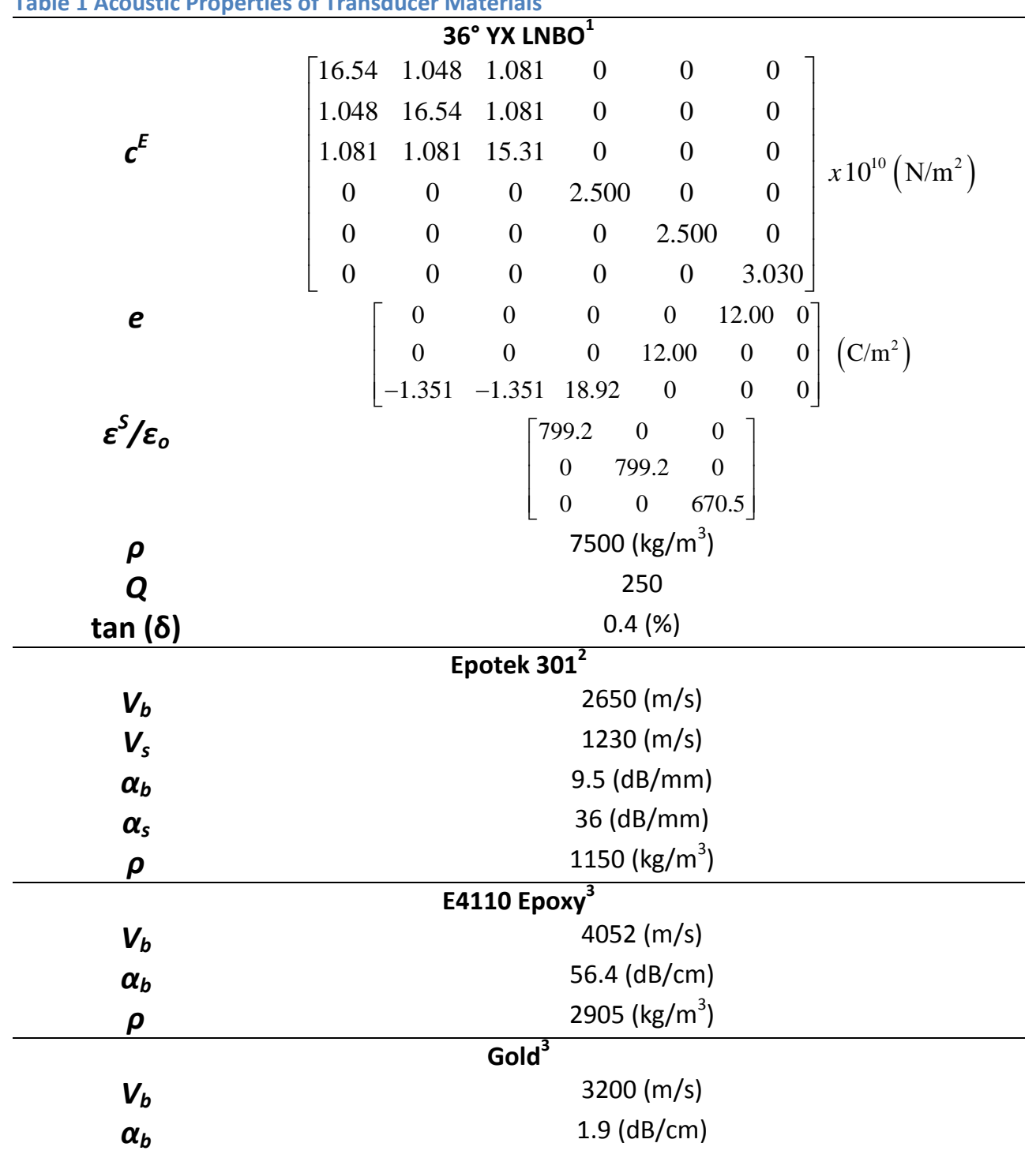




\begin{tabular}{cc}
$\boldsymbol{\rho}$ & $21400\left(\mathrm{~kg} / \mathrm{m}^{3}\right)$ \\
\hline $\boldsymbol{V}_{\boldsymbol{b}}$ & Fused Silica $^{3}$ \\
$\boldsymbol{\alpha}_{\boldsymbol{b}}$ & $5640(\mathrm{~m} / \mathrm{s})$ \\
$\boldsymbol{\rho}$ & $0.1(\mathrm{~dB} / \mathrm{cm})$ \\
\hline
\end{tabular}

${ }^{1}$ The elasticity matrix is given in IEEE format as $[x, y, z, y z, x z, x y]$.

${ }^{2}$ Measured at $30 \mathrm{MHz}$

${ }^{3}$ Measured at $5 \mathrm{MHz}$

\section{Energy and Acoustic Radiation Force}

In the microchannel the pressure field results from the superposition of standing waves in the $z$-direction and waves in the $x$-direction that are inherently weaker due to lateral coupling. The overall pressure field is calculated using the 2D FEM method for a nonviscous fluid. The time averaged energy density $\left(\mathrm{J} / \mathrm{m}^{3}\right)$ in the fields is then split into the kinetic and potential which are given by,

$$
\begin{aligned}
& \left\langle\bar{E}_{k}(x, y)\right\rangle=\frac{\rho_{f}}{2}\left\langle\boldsymbol{v}^{2}(x, y)\right\rangle \\
& \left\langle\bar{E}_{p}(x, y)\right\rangle=\frac{1}{2 \rho_{f} c_{f}^{2}}\left\langle|p(x, y)|^{2}\right\rangle
\end{aligned}
$$

Using these expressions, the force due to a local plane wave acting on a spherical particle in a fluid can be derived from the field potential $U\left(\mathrm{~J} / \mathrm{m}^{3}\right)$ in $2 \mathrm{D}$ as [12],

$$
\begin{aligned}
& \langle U\rangle=2 \pi r^{3} \rho_{f}\left(\frac{f_{1}}{3 \rho_{f} c_{f}^{2}}\left\langle|p(x, y)|^{2}\right\rangle-\frac{f_{2}}{2}\langle v(x, y)\rangle^{2}\right) \\
& f_{1}=1-\frac{\rho_{f} c_{f}^{2}}{\rho_{s} c_{s}^{2}} \quad f_{2}=\frac{2\left(\rho_{s}-\rho_{f}\right)}{2 \rho_{s}+\rho_{f}} \\
& \langle F(x, y)\rangle=-\nabla\langle U(x, y)\rangle
\end{aligned}
$$

where $\rho_{s}$ and $c_{s}$ are the particle density $\left(\mathrm{kg} / \mathrm{m}^{3}\right)$ and acoustic speed $(\mathrm{m} / \mathrm{s})$ of the particle material. This expression is valid for all waves except progressive plane waves, giving the acoustic radiation force $(\mathrm{N})$ acting on microparticles in the fluid. Other effects such as acoustic streaming, gravity, buoyancy, particle interactions, and particles near boundaries are ignored in the derivation of Eq. (0.17).

\section{Thermal Analysis of Acoustic Transducer Array}

To ensure cell lysis is dominated by an acoustic process it is crucial to determine the thermal variation in the fluid and the associated heating of the composite structure. This was accomplished using a multiphysics model that coupled the Navier-Stokes incompressible flow to a convection and conduction model. The fluid flow in the microchannel was described by, 


$$
\begin{aligned}
& \rho(\boldsymbol{u} \cdot \nabla) \boldsymbol{u}+\nabla p-\nabla \cdot \eta\left(\nabla \boldsymbol{u}+(\nabla \boldsymbol{u})^{T}\right)=0 \\
& \nabla \cdot \boldsymbol{u}=0
\end{aligned}
$$

where $u$ is the fluid velocity vector, $\eta$ is the viscosity, $\rho$ is the density, and $p$ is the pressure. By accounting for energy transport through the entire structure, the heat carried by the fluid region, and the heat loss to the ambient media, the steady state thermal distribution can be computed.

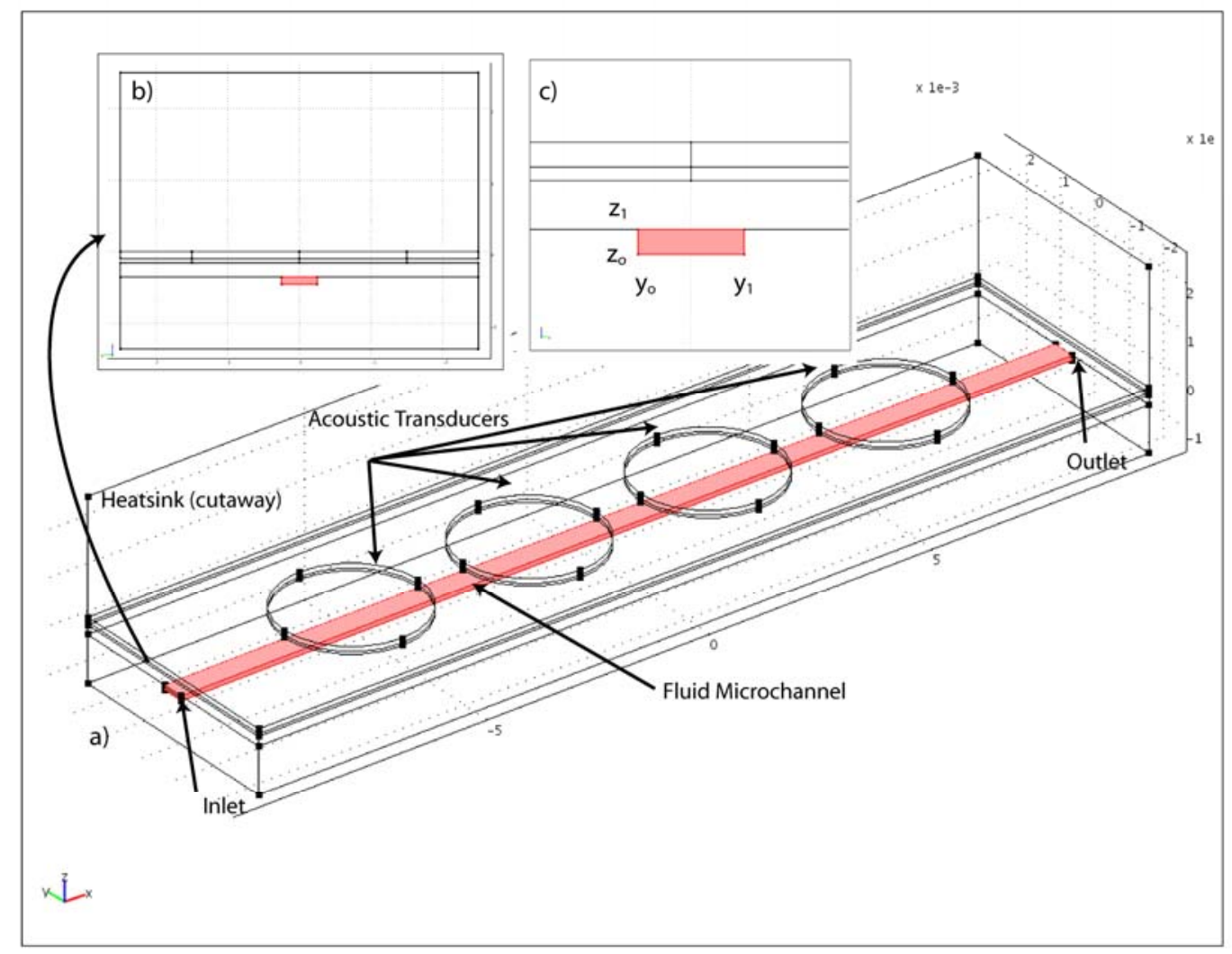

Figure 1 Geometry of the acoustic lysing array. a) The acoustic transducers were modeled as a heat flux discontinuities where the surface of the transducer in contact with the fused silica was given an inward heat flux $Q_{0}$. b) Depicts the left hand side of the modeled geometry where the laminar flow condition was established [Eq.(1.20)]. The fluid input (red region) which was modeled as a fully developed laminar condition. c) The detailed view shows the position of the fluidic channel aligned along the central region of the acoustic transducers.

The stationary thermal transport of convection and conduction was modeled using

$$
\nabla \cdot(-k \nabla T)=Q-\rho C_{p} u \cdot \nabla T
$$

where $C_{p}$ is the specific heat capacity, $\rho$ is the density, $T$ is the temperature, and $Q$ is a sink or source term. For the fully developed laminar flow condition, the following expression was used at the input port,

$$
u=16 u_{\max } \frac{\left(z-z_{o}\right)\left(z_{1}-z\right)\left(y-y_{o}\right)\left(y_{1}-y\right)}{\left(z_{1}-z_{o}\right)^{2}\left(y_{1}-y_{o}\right)^{2}}
$$


where $y$ and $z$ are the position within the channel, $y_{o}$ and $z_{o}$ define the beginning of the channel, and $y_{1}$ and $z_{1}$ defined the extent of the microchannel. At the outlet the heat is dominated by convection thus,

$$
k \nabla T \cdot n=0
$$

With an inward heat flux boundary condition for the piezoelectric transducers,

$$
\begin{aligned}
& -n \cdot\left(q_{1}-q_{2}\right)=q_{o} \\
& q_{i}=-k_{i} \nabla T_{i}
\end{aligned}
$$

The pressure condition was modeled as

$$
p_{o}=0
$$

and no slip at the microfluidic boundaries,

$$
u=0
$$

To couple the maximum velocity in the microchannel to the flow rate the following expression was used,

$$
f_{\text {rate }}[\mu \mathrm{l} / \mathrm{min}]=\text { fluid_velocity }[\mathrm{cm} / \mathrm{s}] \cdot 6 \cdot 10^{4} A_{c s}[\mathrm{~cm}]
$$

\section{B. Transmission Line Method}

For rapid modeling of piezoelectric and non-piezoelectric composites, the transmission line formalism offers significant advantages in computational speed. In the $3 \times 3$ matrix formalism, forces and potential difference across the nth layer relates to the velocities and current at the nth layer. An electrical port defines a voltage across the thickness of a piezoelectric slab with two acoustic ports on each face of the piezoelectric plate. In Figure 2 a single layer $\mathrm{n}$ is shown for a general acoustic transducer which may be piezoelectric or non-piezoelectric.

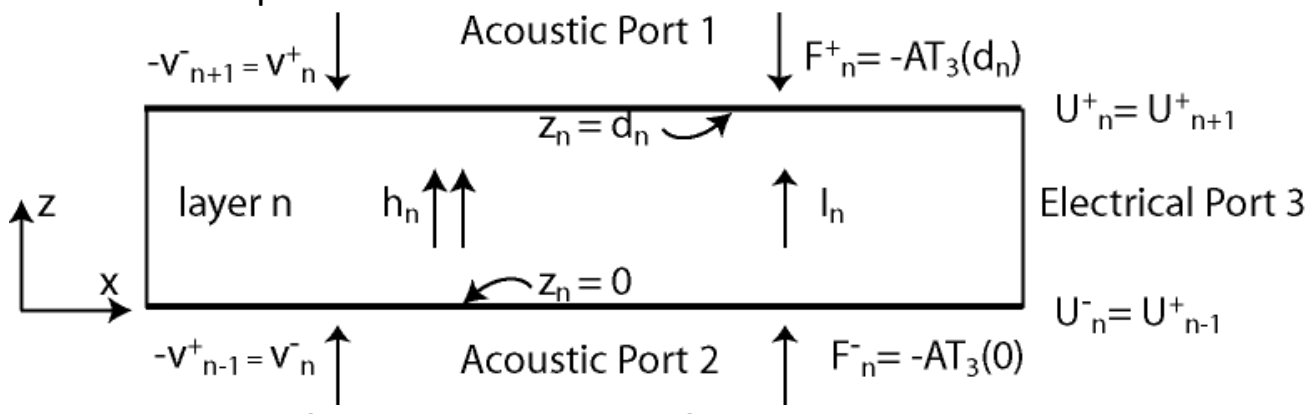

Figure 2 Physical parameters of the nth piezoelectric layer of the transducer which is represented by a three port circuit. The 1-D model assumes that the total thickness of the multilayer transducer is $<10 \%$ of any lateral dimension. The velocity, force, potential, and current at the acoustic ports are $v_{n}, F_{n}, U_{n}$, and $I_{n}$. $h_{n}$ is the polarization of the material of layer $n$.

For a single piezoelectric layer, the forces at the interface can be expressed as

$$
\begin{aligned}
& F_{1}=-A T\left(z_{1}\right)=Z A\left(a e^{-j k z_{1}}-b e^{j k z_{1}}\right)-j \frac{h}{\omega} I \\
& F_{2}=-A T\left(z_{2}\right)=Z A\left(a e^{-j k z_{2}}-b e^{j k z_{2}}\right)-j \frac{h}{\omega} I
\end{aligned}
$$


The goal is to express the $F_{1}$ and $F_{2}$ in terms of the incoming velocities $v_{1}$ and $v_{2}$ at the faces of the layer,

$$
\begin{aligned}
& v_{1}=v\left(z_{1}\right)=a e^{-j k z_{1}}+b e^{j k z_{1}} \\
& v_{2}=v\left(z_{2}\right)=-a e^{-j k z_{2}}-b e^{j k z_{2}}
\end{aligned}
$$

The voltage applied to the faces is determined by integrating the electric field $\mathrm{E}$ across the thickness of the transducer,

$$
\begin{aligned}
& U=\int_{z_{1}}^{z_{2}} E d z=-\int_{z_{1}}^{z_{2}} h \frac{\partial u}{\partial z}-\frac{D}{\varepsilon^{S}} d z=\frac{h}{j \omega}\left(v_{1}+v_{2}\right)+\frac{I}{j \omega C_{o}} \\
& C_{o}=\frac{\varepsilon^{S} A}{d}
\end{aligned}
$$

By casting in matrix form the expressions $(0.26),(0.27)$, and $(0.28)$ were combined to give

$$
\left[\begin{array}{l}
F_{1} \\
F_{2} \\
U
\end{array}\right]=\left[\begin{array}{ccc}
-\frac{Z_{o}}{\tanh (\gamma d)} & \frac{Z_{o}}{\sinh (\gamma d)} & -\frac{h}{j \omega A} \\
-\frac{Z_{o}}{\sinh (\gamma d)} & \frac{Z_{o}}{\tanh (\gamma d)} & -\frac{h}{j \omega A} \\
\frac{h}{j \omega} & -\frac{h}{j \omega} & \frac{1}{j \omega C_{o}}
\end{array}\right]\left[\begin{array}{c}
v_{1} \\
v_{2} \\
I
\end{array}\right]
$$

From this key result $[13,14]$ the electric impedance was derived for a layered system containing a single piezoelectric element by solving for $U$ as a function of $I$,

$$
Z_{e}=\frac{1}{j \omega C_{o}}+\frac{h^{2}}{\omega^{2} A}\left[\frac{2[\cosh (\gamma d)-1] Z_{t}+\left(Z_{L}+Z_{R}\right) \sinh (\gamma d)}{\left(Z_{L} Z_{R}+Z_{t}^{2}\right) \sinh (\gamma d)+Z_{t}\left(Z_{L}+Z_{R}\right) \cosh (\gamma d)}\right]
$$

where $\gamma$ is the propagation constant, $Z_{L}$ is the total impedance loading on the left, $Z_{R}$ is the total impedance loading on the right, $A$ is the cross sectional area, $h$ is the stress piezoelectric constant (i.e. $e_{33} / \varepsilon_{33}$ ), and $Z_{t}$ is the acoustic impedance of the transducer. The acoustic attenuation coefficient $\alpha$ was taken to vary as frequency to the nth power,

$$
\gamma=\alpha+j \beta=\alpha_{o}\left(\frac{f}{f_{o}}\right)^{n}+\frac{j \omega}{v}
$$

where $\mathrm{n} \approx 1.5$. For electrical loss the complex dielectric constant was defined as

$$
\varepsilon_{33}^{s}=\varepsilon_{r} \varepsilon_{o}\left(1-j \tan \left(\delta_{e}\right)\right)
$$

where $\delta_{e}$ is the dielectric loss tangent of the material, $\varepsilon_{r}$ is the relative dielectric constant.

To compute the electrical impedance of a system comprising a single piezoelectric element, the loading impedances on each face of the transducer $\left(Z_{L}, Z_{R}\right)$ must be calculated for the composite layers. This expression for successively transforming impedances layer by layer is given by 


$$
Z^{+}=Z_{\text {in }} \frac{Z_{\text {Load }} \cosh (\gamma d)+Z_{\text {in }} \sinh (\gamma d)}{Z_{\text {in }} \cosh (\gamma d)+Z_{\text {Load }} \sinh (\gamma d)}
$$

where $Z_{\text {Load }}$ is the impedance of the loading layer and $Z_{\text {in }}$ is the impedance of the intermediate layer. The layer impedances are a function of frequency and are computed as

$$
Z(\omega)=\frac{j \omega \rho}{\gamma}
$$

Another useful set of relations are the acoustic velocities at the faces of the transducer which are given by

$$
\begin{aligned}
& v_{L}=\left[\frac{h}{j \omega A} \frac{Z_{o}(1-\cosh (\gamma a))-Z_{R} \sinh (\gamma a)}{\left(Z_{L} Z_{R}+Z_{o}^{2}\right) \sinh (\gamma a)+Z_{o}\left(Z_{L}+Z_{R}\right) \cosh (\gamma a)}\right] \frac{V}{Z_{e}} \\
& v_{R}=\left[\frac{-h}{j \omega A} \frac{Z_{o}(1-\cosh (\gamma a))-Z_{L} \sinh (\gamma a)}{\left(Z_{L} Z_{R}+Z_{o}^{2}\right) \sinh (\gamma a)+Z_{o}\left(Z_{L}+Z_{R}\right) \cosh (\gamma a)}\right] \frac{V}{Z_{e}}
\end{aligned}
$$

By knowing the acoustic impedances loading each face of the transducer, the total radiated power can be calculated using

$$
P_{\text {total }}=P_{L}+P_{R}=\frac{A}{2}\left[\operatorname{Re}\left(Z_{L}\right)\left|v_{L}\right|^{2}+\operatorname{Re}\left(Z_{R}\right)\left|v_{R}\right|^{2}\right]
$$

For the subsequent 3D FEM analysis the amount of energy converted into heat is required for the boundary condition between the transducer and silver epoxy/aluminum heat sink. To compute this value, it is assumed that electrical and mechanical phenomena are the dominant loss mechanisms. From conservation of energy,

$$
\begin{aligned}
& P_{m}+P_{e}=\frac{1}{2} \operatorname{Re}\left(Z_{e}\right)|I|^{2}-P_{\text {total }} \\
& P_{e}=\frac{1}{2} R_{p}|I|^{2}
\end{aligned}
$$

where $P_{m}$ and $P_{e}$ are the mechanical and electrical loss in watts. $R_{p}$ is the combined internal electrical losses which is the real value of the electrical impedance at the frequency where the displacements are 0.

The solving procedure requires that the velocities, thicknesses, densities and attenuations are known for each layer. First the propagation constant $\left(\gamma_{n}\right)$ for each layer is computed followed by computing (0.34) for each layer. Using $(0.33)$ the impedance loading the transducer are computed by successive substitution and combined with $(0.30)$ to compute the total electrical impedance of the loaded transducer. The advantage of one dimensional transmission line models is they permit rapid design and optimization of transducers that have a large number of layers. Moreover, optimization algorithms can be used to enhance power output, impedance matching, and frequency response. 


\section{Materials and Methods}

\section{A. Transducer Fabrication}

The actuators were fabricated using 360 Y LNBO (Boston Piezo-Optics, Bellingham, MA) substrates and were $50 \mu \mathrm{m}$ thick and $3 \mathrm{~mm}$ in diameter. Gold ( $\mathrm{Au}$ ) electrodes were deposited by the manufacturer and were $2000 \AA$ thick with $0.2 \mathrm{~mm}$ hold back from the edge of the substrates. Electrical connections to the piezoelectric substrates were made by depositing gold electrodes and contact pads onto fused silica substrates $(20.5 \mathrm{~mm} \mathrm{x}$ $44.8 \mathrm{~mm} \times 0.5 \mathrm{~mm}$ ) obtained from University Wafer (South Boston, MA) (Figure 3). Briefly, a positive photoresist AZ 5214 (Hoechst Celanese, Corp., Branchburg, NJ) was applied to the fused silica substrates using a photoresist spinner at $4500 \mathrm{rpm}$ for $30 \mathrm{~s}$. The substrates were then baked at 110 으 for $60 \mathrm{~s}$ and exposed for $15 \mathrm{~s}$ to a UV lamp (intensity of $110 \mu \mathrm{W} / \mathrm{cm}^{2}$ at $320 \mathrm{~nm}$ ) from a Carl Suss MJB3 mask aligner. The fused silica substrates were developed for $35 \mathrm{~s}$ in Microposit 351 photoresist developer (Hoechst Celanese Corp., Branchburg, NJ) diluted 1:4 in deionized water.
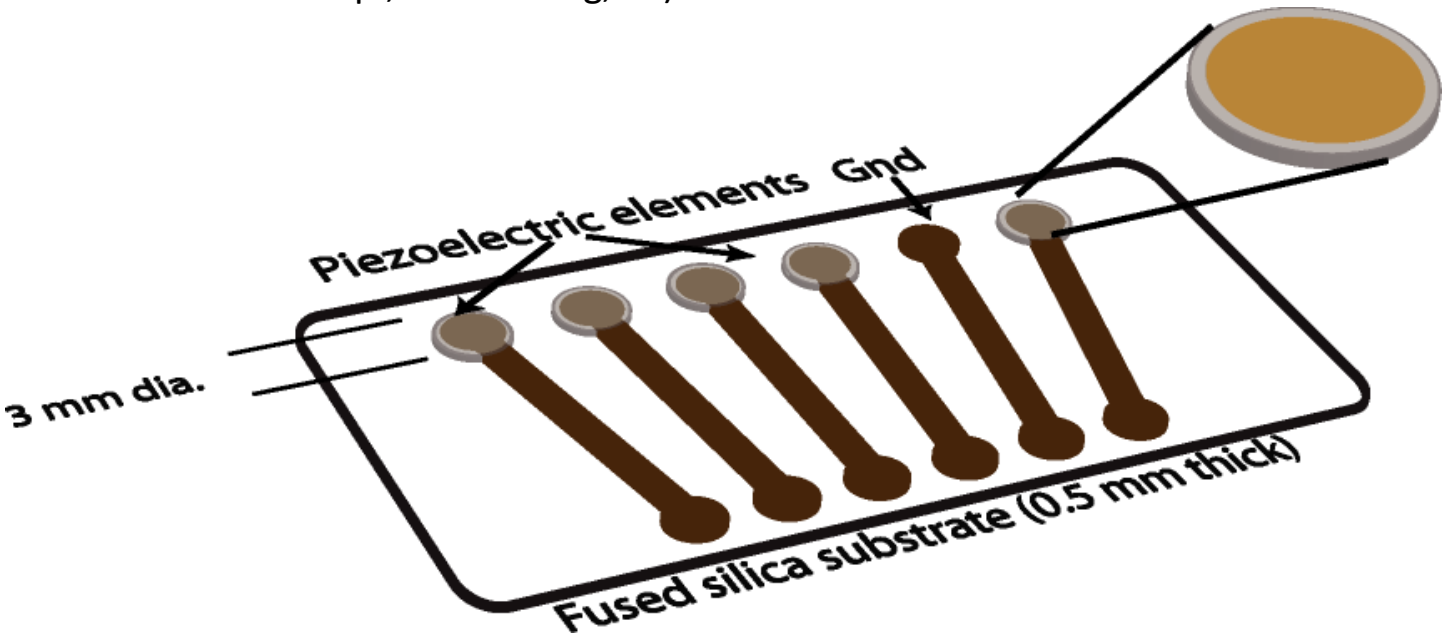

Figure 3 BAW transducer array. The first four transducer elements are used for cell lysis and the final element is for mixing solutions in the microchannel. This transducer array couples to the microchannels through a fused silica boundary, where remote actuation of the fluid is accomplished by the propagation of acoustic waves. Each transducer is $3 \mathrm{~mm}$ in diameter. The transducer elements shown in brown are addressed by $\mathrm{Au} / \mathrm{Cr}$ electrodes patterned using a shadow mask process.

The piezoelectric substrates were first bonded to gold contact pads patterned on the fused silica substrate using a $100 \mu \mathrm{m}$ thick layer of Epotek 4110 conductive epoxy (Epotek Technology, Billerica, MA) (Figure 4). The placement of the conductive epoxy was controlled by using a 4 mil Mylar masking layer cut using a VersaLaser VLS3.50 system (Universal Laser Systems, Scottsdale, AZ). The E4110 epoxy was cured at 150ㄷ C for $15 \mathrm{~min}$. The transducers were individual bonded and checked for short circuits before the encapsulation procedure. A confinement Mylar mask was cut on the laser and attached directly to the transducer Mylar mask. The backside of each transducer was masked during the encapsulation process using PDMS cylinders $(2.5 \mathrm{~mm}$ dia., $3 \mathrm{~mm}$ height). The encapsulating epoxy was prepared using 5:20 (w/w) mixture of Epotek 


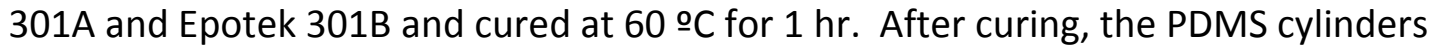
were removed and the region was filled with Epotek 4110 conductive epoxy. An Al heatsink was bonded above the transducers using a 10:1 (w/w) mixture of Epotek 4110A and Epotek 4110B (Epotek Technology, Billerica, MA) conductive epoxy and cured for 15 $\min$ at $150 \circ \mathrm{C}$ (Figure 4). This heatsink removed heat from the lysing region and acoustic transducers. The Al heat sink was $12 \mathrm{~mm} \times 30 \mathrm{~mm} \times 5 \mathrm{~mm}$ high (not shown).

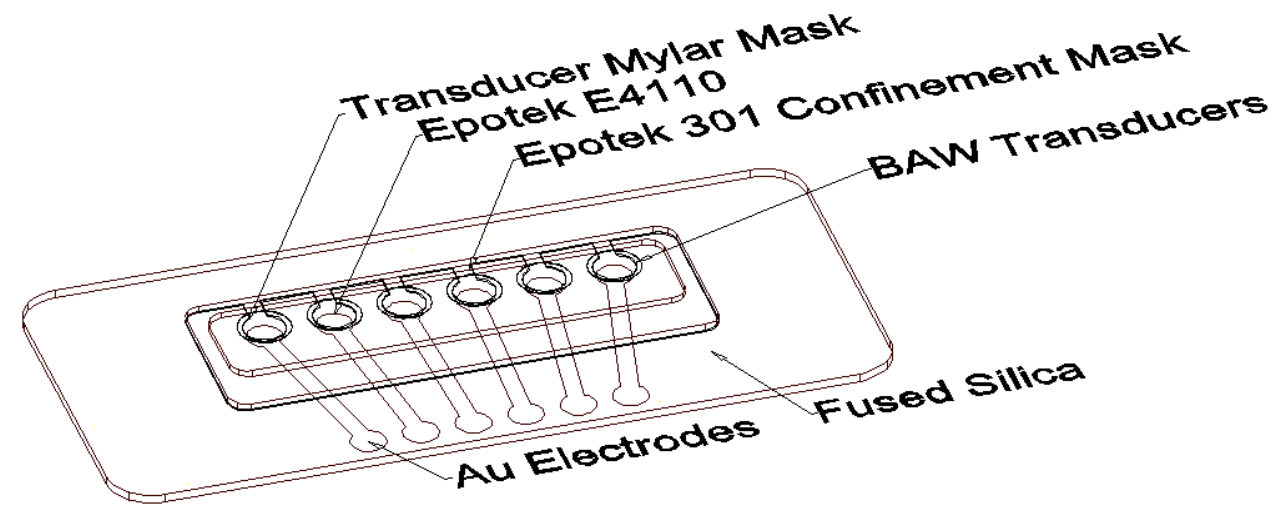

Figure 4 Scale drawing of BAW transducer array assembly. Assembly process begins with the application of the transducer Mylar mask to the fused silica substrate. The BAW transducers are bonded to the Au electrodes using Epotek 4110 epoxy. By masking the backside of the transducers, the region defined as the confinement mask is backfilled with Epotek 301 epoxy.

\section{B. Microchannel Fabrication}

\section{PDMS Microfluidic Channel}

Three types of microchannels designs were fabricated to deliver cells to the BAW lysis region. The first design was fabricated from polydimethylsiloxane (PDMS) using an Al mold to cast the microchannel. The mold was fabricated with the negative pattern of the microchannel. Fluid connections were established by casting silicon rubber tubing directly into inlet and outlet of the PDMS microchannels. To achieve this, holes were created in the Al mold at the inlet and outlet of the microchannel which held two copper $(\mathrm{Cu})$ posts $5 \mathrm{~mm}$ high. Silicon rubber tubing was pushed over the Cu posts, preventing PDMS leakage into the silicon rubber tubing. The PDMS microchannel was cast using a 1:10 (v/v) mixture of Sylgard silicon and Sylgard silicone elastomer 184 (Dow Corning Corporation). As a release agent for the cured PDMS microchannel, a 5\% Triton $\mathrm{X}-100$ solution was applied to the Al mold by a flood procedure. The PDMS microchannels were bonded directly to the acoustic BAW transducer. This approach allowed rapid analysis of the lysing efficacy without committing to more advanced microchannel designs.

\section{DNA Binding Paramagnetic Beads Based Cartridge}

These designs were based on $\mathrm{CO}_{2}$ laser processing of Mylar and PMMA layers to create a composite nucleic acid extraction cartridge. The two designs in Figure 5 lyse the cellular samples and immediately capture nucleic acid to the surface of paramagnetic 
beads. The magnetic core of the beads allowed confinement within the microchannel as the buffer was exchanged to remove non-nucleic material. In Figure 5a, the O-ring layer is defined as \#6 which holds a 001 silicone O-ring (I.D. 1/32", O.D. 3/32) (McMaster-Carr, Los Angeles, CA). The microchannels were $100 \mu \mathrm{m}$ in height and $500 \mu \mathrm{m}$ in width. 


Layer 1 FS 25 mil
Layer 2 AMA 4mil
Layer 3 Mylar 14mil
Layer 4 MA 3mil
Layer 6 PMMA 80mil 5 AMA 4mil
Layer 7 AMA 4mil
Cayer 8 PMMA 62mil

\section{Nafion-Based Extraction Cartridges}

For the Nafion system the O-ring layer was \#7 (Figure 5b). A top layer defined by a 62 mil Mylar layer provides strain relief for the $1 \mathrm{~mm}$ O.D. FEP tubing $(0.5 \mathrm{~mm}$ I.D.) (Upchurch Scientific, Oak Hardbor, WA). The stack was aligned using alignment holes in each layer and then compressed in a hydraulic press for 2 minutes at 3 tons. The fused silica layer was bonded separately at lower pressure. This approach provided a complete standalone microchannel that could be removed from the acoustic BAW transducer. The microchannels were $100 \mu \mathrm{m}$ in height and $500 \mu \mathrm{m}$ in width. The electrodes were fabricated by evaporating $200 \AA$ of $\mathrm{Cr}$ and $5000 \AA$ of Au onto $100 \mu \mathrm{m}$ thick Mylar discs. The discs were used to create layers 4 and 6 in Figure 5. During assembly layer $\mathbf{6}$ defined a unique wrap-around electrode that bonded to layer $\mathbf{8}$ and 
was held in place by layer $\mathbf{9}$. This permitted single sided electrical connectivity to the Nafion coated electrodes.

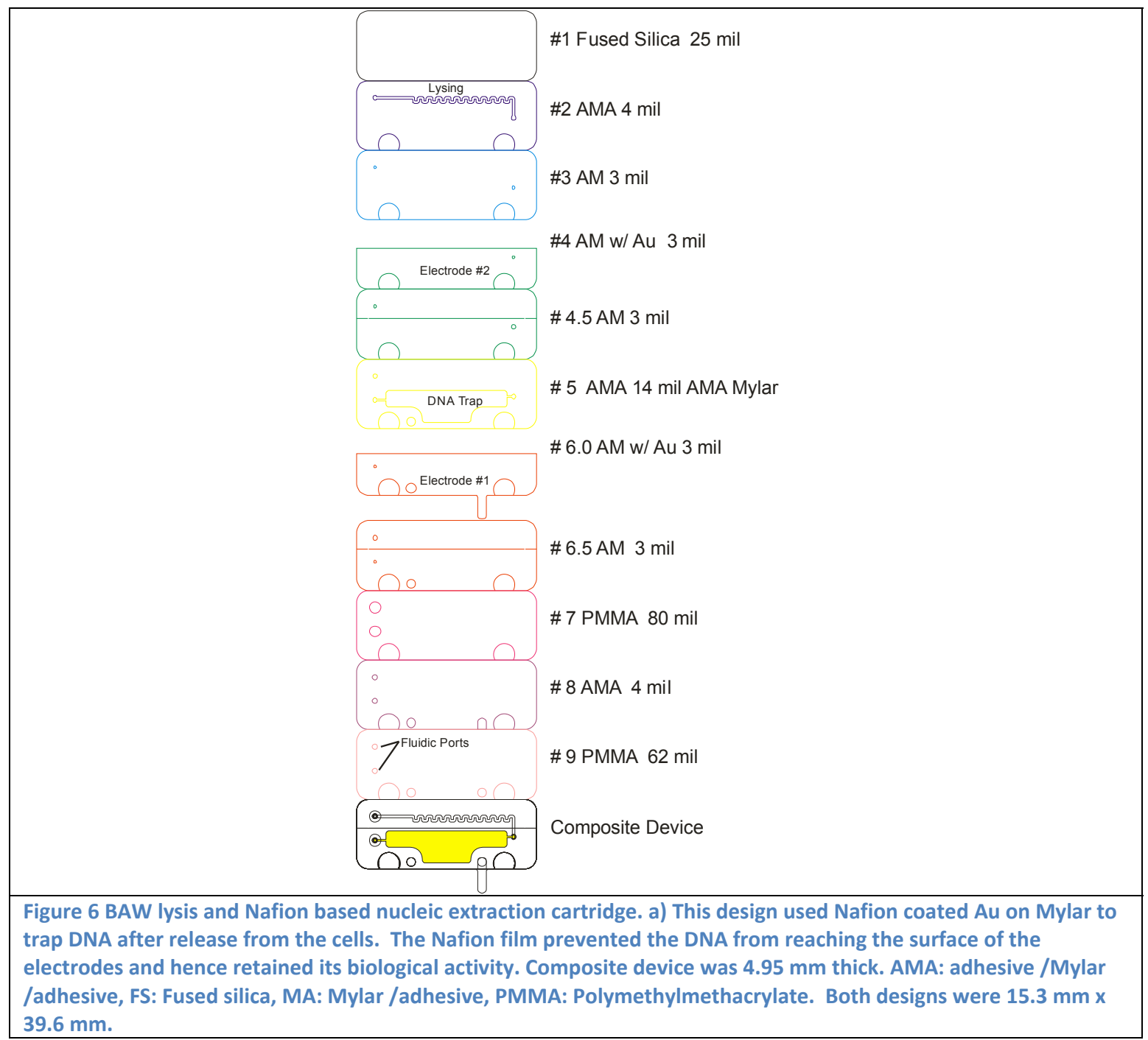

\section{DNA Extraction Using Paramagnetic Beads}

Several commercially available kits employ silica or polystyrene beads with a paramagnetic core for rapid, facile isolation of DNA from a complex mixture of cellular lysate. For example, the ChargeSwitch ${ }^{\circledR}$ gDNA Mini Bacteria Kit manufactured by Invitrogen (Carlsbad, CA) reversibly binds DNA to the bead surface in response to a change in solution $\mathrm{pH}$. Following cell lysis, DNA is bound to the surface of the $1 \mu \mathrm{m}$ ChargeSwitch $^{\circledR}$ magnetic beads by lowering the solution $\mathrm{pH}$ to $<6.5$, which protonates the bead surface for binding with the negatively charged phosphate backbone of the DNA. Unbound contaminants are removed in two wash steps, and the DNA is eluted from the beads by raising the $\mathrm{pH}$ to 8.5 , which deprotonates the bead surface and releases the bound DNA. The paramagnetic core of the beads permits immobilization of the beads within an externally applied magnetic field for reagent exchange without 
sample loss. These kits provide a method for performing high-throughput assays in a reduced volume that is suitable for translation into a microfluidic chip-based format. Modifications to the basic lysis cartridge presented in the previous section were made to integrate ChargeSwitch ${ }^{\circledR}$ beads for DNA extraction and purification following acoustic lysis. Three unique cartridge designs were fabricated to test the recovery of genomic $E$. coli DNA, strain B (Sigma-Aldrich, St. Louis, MO) following the bead-based assay. Each design employed two removable magnets for selective trapping of beads within the channel during the assay. The first design incorporated two 1/4" diameter magnets (KJ Magnetics, Jameson, PA) and relied on passive binding and release of DNA as the reagent solution was passed over the bead reservoir. The second design took advantage of a piezoelectric transducer positioned under the bead reservoir for active mixing of the beads with the reagent solution and to acoustically drive DNA off of the bead surface. In addition, smaller, 1/8" diameter magnets were used for bead trapping within the channel (KJ Magnetics, Jameson, PA). The third cartridge design (Figure 5a) included the features of the second design as well as incorporating a larger bead reservoir with a volume of $25 \mu \mathrm{L}$. Bead loading efficiency for all designs was tested by injecting a bead suspension into the microfluidic channel with the magnets engaged and performing a particle count on the eluent using an Accuri C6 Flow Cytometer (Accuri Cytometers, Ann Arbor, MI). All assay buffers were provided with the ChargeSwitch ${ }^{\circledR}$ gDNA Mini Bacteria Kit, the formulations of which were not disclosed in the product literature. For these experiments, the buffers will be referred to as "binding buffer" ( $\mathrm{pH}$ $<6.5)$, "wash buffer" ( $\mathrm{pH}=7.0)$, and "elution buffer" ( $\mathrm{pH}=8.5)$. For each experiment, $100 \mu \mathrm{L}$ of genomic $E$. coli DNA solution was combined with $300 \mu \mathrm{L}$ of binding buffer and injected into the microfluidic cartridge, followed by $500 \mu \mathrm{L}$ of wash buffer to remove nonspecifically bound impurities. In the last step, $200 \mu \mathrm{L}$ of elution buffer was injected to release bound DNA. The eluent from each step was collected and analyzed for DNA content using a Quant-iT ${ }^{\mathrm{TM}}$ PicoGreen assay purchased from Invitrogen (Carlsbad, CA). PicoGreen is a dye that fluoresces upon binding with double-stranded DNA. The assay was performed in a 96-well plate format using a SpectraMax M2 plate reader (Molecular Devices, Sunnyvale, CA) and the data fitted to a standard curve generated from known concentrations of DNA.

\section{DNA Nafion-Based Extraction}

A second model for utilizing applied voltage for electrostatic extraction of DNA was developed. Using an electric field nucleic acid extraction concept presented by Lee et al. [10], an impoved microfluidic cartridge incorporating a Nafion-coated gold electrode was fabricated (Figure 6). The cartridges were manufactured in a fashion similar to the designs described previously, with the addition of a Nafion-coated gold electrode with an available surface area of $141 \mathrm{~mm}^{2}$ for DNA binding. For each experiment, $50 \mu \mathrm{L}$ of $E$. coli genomic DNA in $10 \mathrm{mM}$ TE buffer (10 mM Tris-Cl pH 7.4, 1 mM EDTA pH 8.0) was injected into the microfluidic channel at a $50 \mu \mathrm{L} / \mathrm{min}$. flow rate. Once the solution entered the channel, a positive voltage was applied to the electrode using an Agilent E3630A Triple Output DC Power Supply to bind DNA. The voltage was applied until all of the solution has run through the channel, at which time, the power supply was turned 
off. $50 \mu \mathrm{L}$ of $10 \mathrm{mM}$ TE buffer was then loaded into the channel at a $50 \mu \mathrm{L} / \mathrm{min}$. flow rate, and a positive voltage was once again applied to the electrode to keep the DNA bound until the entire volume of buffer had exited the channel. For the elution step, 50 $\mu \mathrm{L}$ of $10 \mathrm{mM}$ TE buffer was loaded into the channel at a $50 \mu \mathrm{L} / \mathrm{min}$. flow rate and a negative voltage was applied to release bound DNA from the electrode. The eluent from each step was collected and analyzed for DNA content using the Quant-iT ${ }^{\mathrm{TM}}$ PicoGreen assay. The voltages for binding and elution of DNA were chosen based on electric field calculations and ranged from +/- $2.5 \mathrm{~V}$ to $+/-7.92 \mathrm{~V}$.

\section{Nucleic Acid Extraction Approaches}

After successfully demonstration of the BAW lysing system, the focus was to integrate a nucleic acid extraction region in the cartridge. The extraction method had to satisfy several criteria: 1) suitable for microformat, 2) compatible fluidic requirements for interfacing with the lysis region, and 3 ) fast extraction time on the scale of a few minutes. In Figure 7 the nucleic acid extraction approaches are outlined. Since the solgel silica bead matrix (Figure 7a) is fabricated in a glass microchannel, the corresponding lysis components (i.e. serpentine microchannel and fluidic ports) were fabricated in glass. The cartridge implementations in Figure $7 \mathrm{~b}$ and $\mathrm{c}$ were fabricated using layers of Mylar, acylic, and fused silica, which dramatically reduced the design and fabrication time.

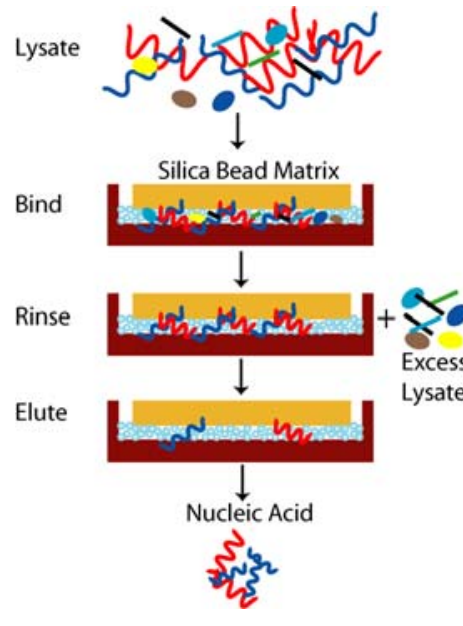

a) b)

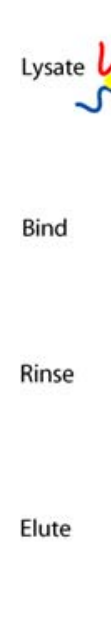

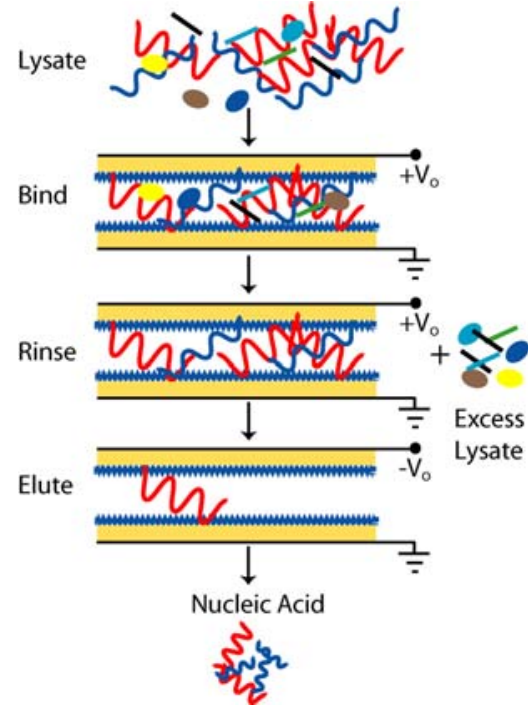

c)

Figure 7 Nucleic acid extraction methods for a complete single cartridge solution. a) Sol-gel silica bead matrix based extraction. The silica matrix was fabricated by injecting or patterning sol-gel matrix containing silica beads, b) Magnetic bead based nucleic acid extraction. Nucleic acid binding magnetic beads $\left(10^{6}\right.$ beads $\left./ \mathrm{ml}\right)$ were mixed with raw cellular samples prior to lysis. A pH shift from 6.5 to 8.5 eluted the purified nucleic acid, c) Nafion coated electrode based extraction. A Nafion film was applied to gold electrodes on a Mylar substrate to electrostatically bind and release nucleic acid. 


\section{Cell Culture}

\section{Cell Sample preparation}

Escherichia coli cultures (ATCC 10798) were provided by Susan Brozik and were cultivated in BBL ${ }^{\text {TM }}$ Mueller Hinton II Broth, Cation Adjusted (Becton Dickinson and Company, Sparks, MD), following standard methods. Organisms were incubated at 37으 and 120 RPM in a shaking water bath, and growth was followed by monitoring the change in optical density of the suspension at $600 \mathrm{~nm}\left(\mathrm{OD}_{600}\right)$ using a DU-800 spectrophotometer (Beckman Instruments, Inc., Fullerton, California). Cells were grown to log phase, harvested, washed twice in 1X PBS (137 mM NaCl, $2.7 \mathrm{mM} \mathrm{KCl}, 4.3 \mathrm{mM}$ $\mathrm{Na}_{2} \mathrm{HPO}_{4} \cdot 7 \mathrm{H}_{2} \mathrm{O}, 1.5 \mathrm{mM} \mathrm{KH}_{2} \mathrm{PO}_{4}$ ) and resuspended in $1 \mathrm{X}$ PBS at an average cell density of $5 \times 10^{8} \mathrm{CFU} / \mathrm{mL}$. The stock suspension was kept refrigerated and all experiments were completed within two hours of the initial sample preparation.

\section{Sample Sonication using a Commercial System}

Approximately $50 \mu \mathrm{L}$ of cell suspension was loaded into a $1 \mathrm{cc}$ syringe and injected into the device through a short length of $0.030 \mathrm{~mm}$ ID/0.065 $\mathrm{mm}$ OD flexible silicone tubing (VWR, West Chester, PA) using a syringe pump (KD Scientific, Holliston, MA). A second syringe pump (Harvard Apparatus, Holliston, MA) was used to withdraw the sample from the channel through a second length of tubing. Later modifications to the microfluidic cartridge increased the channel height to make pumping liquid out of the channel unnecessary. An 8648A synthesized signal generator (Agilent Technologies, Santa Clara, CA) and an RF power amplifier (Bell Electronics NW, Inc., Kent, WA) were used to drive the piezoelectric transducers for all experiments. Power input was varied from $0-200 \mathrm{~mW}$ at $100 \%$ duty cycle and sample flow rates were varied from $10-25$ $\mu \mathrm{L} / \mathrm{min}$. to maximize lysing efficiency while avoiding excessive heat generation and acoustic cavitation during the experiments. The microfluidic system was flushed with $0.5 \mathrm{~mL}$ distilled water between experiments. Additional flow-only experiments were performed to insure that lysis was not occurring through a shearing mechanism during the sample injection process.

Positive controls were performed using a $20 \mathrm{kHz}$ Sonicator 3000 bench top sonication system (Misonix, Farmingdale, NY) using both the acoustic finger and the acoustic cup configurations. For the acoustic finger experiments, a 1/16" tip was inserted into a $2 \mathrm{~mL}$ microcentrifuge tube containing a $1 \mathrm{~mL}$ sample using $12 \mathrm{~W}$ of applied power and an $8 \%$ duty cycle as recommended by the manufacturer (total process time $=60 \mathrm{sec}$.). During the experiment, the sample container was submerged in an ice bath to dissipate heat generated by the acoustic transducer. In the acoustic cup configuration, $100 \mu \mathrm{L}$ of sample in a closed $2 \mathrm{~mL}$ microcentrifuge tube was submerged into a water-filled cup, the base of which encapsulated a 1.8" flat acoustic transducer. During the experiment, $58.5 \mathrm{~W}$ of power was applied at a $100 \%$ duty cycle (total process time $=21.5$ minutes). Heat generated by the transducer was dissipated by circulating the water through a chiller for the duration of the experiment. It is worthy to note that the manufacturer's recommendation included the addition of mechanical or chemical lysing agents to 
improve lysing efficacy. To remain consistent with the microfluidic BAW lysing system protocol, no such agents were used.

\section{ATP Assay}

Lysing efficacy was initially assessed by measuring ATP liberated from treated samples and comparing it to the measured ATP of untreated samples. A firefly luciferase-based ATP Determination Kit was purchased from Molecular Probes (Eugene, OR), and performed in a 96-well microplate format. Luminescence measurements were taken using a Berthold-Mithras LB 940 microplate reader (Berthold Technologies, Oak Ridge, TN). ATP concentrations were calculated by fitting experimental data to a standard curve generated with known concentrations of ATP. Linearity of light output vs. ATP concentration was confirmed using ATP standard solutions over the range of $10^{-6}-10^{-10}$ M.

\section{Cell Viability}

The efficacy of the sonication platforms was further measured by comparing viable cell counts before and after treatment using the method of Miles and Misra for plate counts [15]. A serial 10-fold dilution of the sample was performed in sterile 1X PBS within ten minutes of the lysis experiments. Aliquots of $20 \mu \mathrm{L}$ were spotted in triplicate onto Difco $^{\text {TM }}$ plate count agar (Becton Dickinson and Company, Sparks, MD) and incubated at room temperature for 24 hours. The percentage of viable cells was calculated using $100\left(C_{0}-C_{\text {treated }}\right) / C_{0}$, where $C_{0}$ is the average viable cell count prior to sonication and $\mathrm{C}_{\text {treated }}$ is the average viable cell count after sonication.

\section{Results and Discussion}

\section{A. Transmission Line Model of Bulk Acoustic Wave Transducer Array}

The first task was to design a bulk acoustic wave (BAW) transducer to couple sufficient acoustic energy into a fluid filled region to disrupt the cellular membrane. Secondly, extend this design and fabricate a BAW transducer array that could reversible couple to a microchannel cartridge. The microchannel cartridge would provide the necessary fluidics to mix the lysate with one of the following DNA extraction formats: magnetic core beads that reversibly bind DNA or a charge based capture and release technique [10]. Reversible coupling to the BAW array was crucial to permit reuse of the transducer assembly while allowing disposal of the contaminated fluidic cartridges. Thus, the interface between the acoustic transducers and the microfluidic channel was critical to obtain the best possible acoustic coupling and permit reusability of the transducer array. A significant modeling effort was undertaken to investigate the physical parameters to reduce acoustic loss through the boundaries while permitting fluid movement through the microchannels. The modeled geometries of the unloaded and loaded transducer are shown in Figure $8 \mathrm{a}$ and $\mathrm{b}$. 
a)
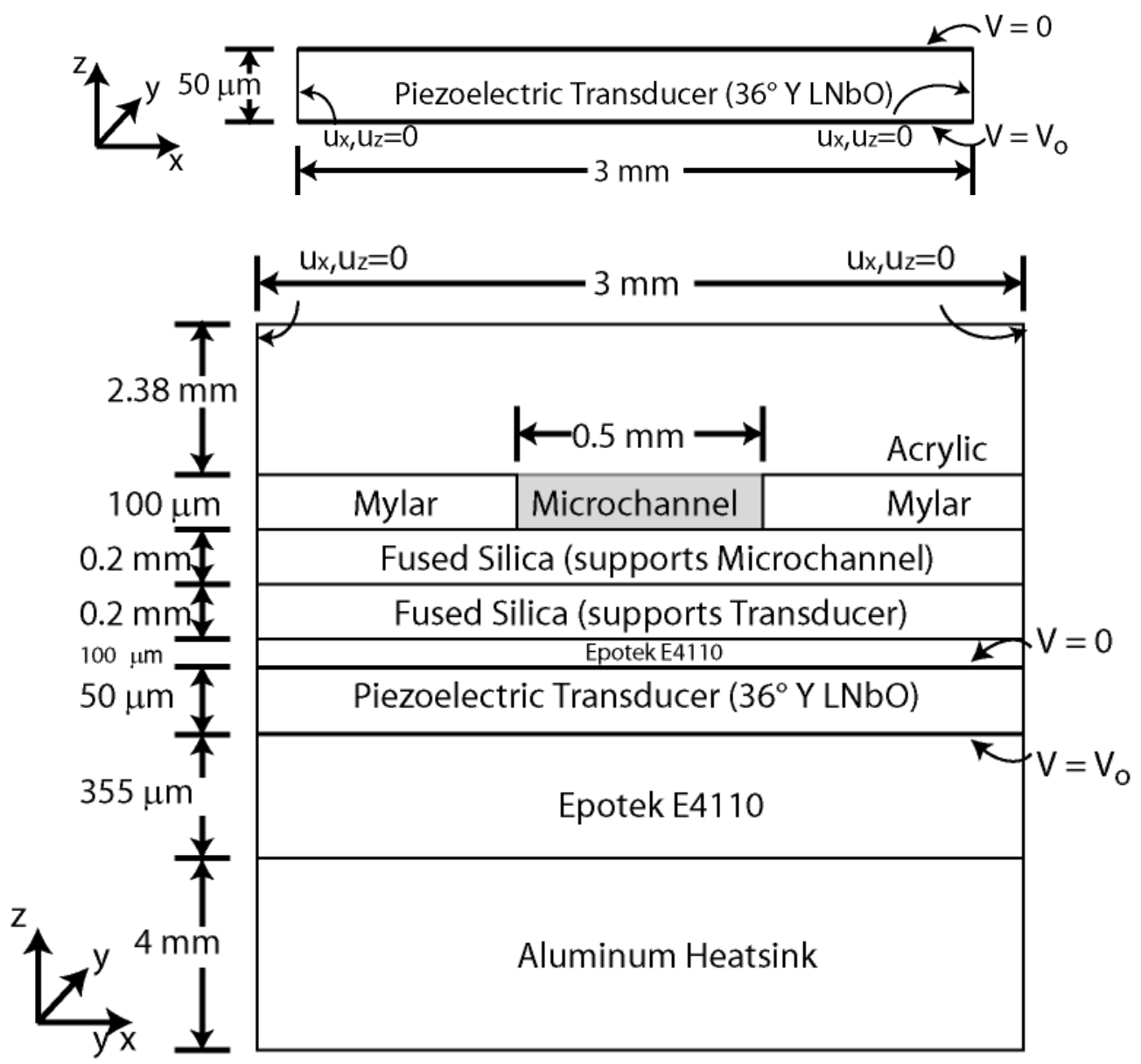

b)

Figure 8 Cross-section of BAW transducer and composite structure. a) Unloaded transducer. b) Composite model of the transducer which shows the required layers to permit removal of the microchannel cartridges from the BAW transducer. For the impedance calculations $V=1 V$. 


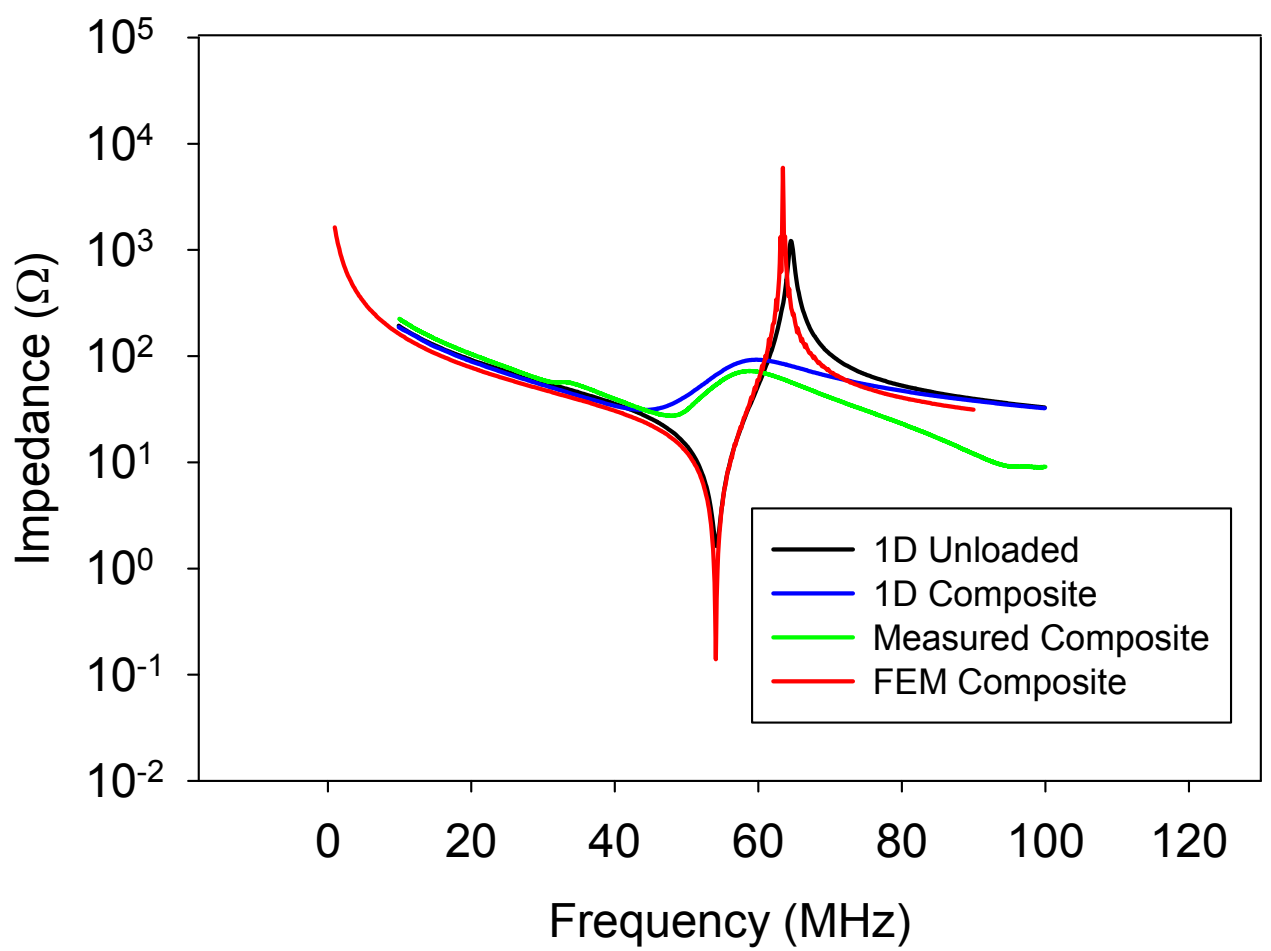

Figure 9 Impedance response of 36 Y lithium niobate transducer using the 1D Transmission line method. Though the unloaded transducer exhibits excellent resonance near $55 \mathrm{MHz}$, the impedance was far from optimal Z(55MHz) $=1.10-\mathrm{j} 7.15 \Omega$. For the composite transducer, $\mathrm{Z}(55 \mathrm{MHz})=44.67-\mathrm{j} 16.9 \Omega$, which a much better match to a $50 \mathrm{Ohm}$ system. Perfect matching was undesirable since this would require exquisite tracking of the maximal drive frequency. Therefore, broadband behavior was required for this application.

In Figure 9 the impedance response is shown for the unloaded and loaded transducer based on the 1D transmission line and 2D FEM model. Transmission line approaches were significantly faster computationally, providing rapid insight into the impact of various layers on the transducer. One disadvantage of this method is the cross-sectional area is assumed to be a constant from layer to layer. Despite the limitations of a 1D analysis, several key parameters can be extracted from this model: the return loss (S11), the acoustic velocities at the transducer faces, and the radiated acoustic power. In the next section, a more realistic model was developed using a 2D FEM and 2D axially symmetric analysis which correctly accounted for changes in area from layer to layer. Validity of this model was performed by assuming lossless conditions to determine the resonance and anti-resonance frequencies, which were used to compute the electromechanical coupling constant (Figure 11). 


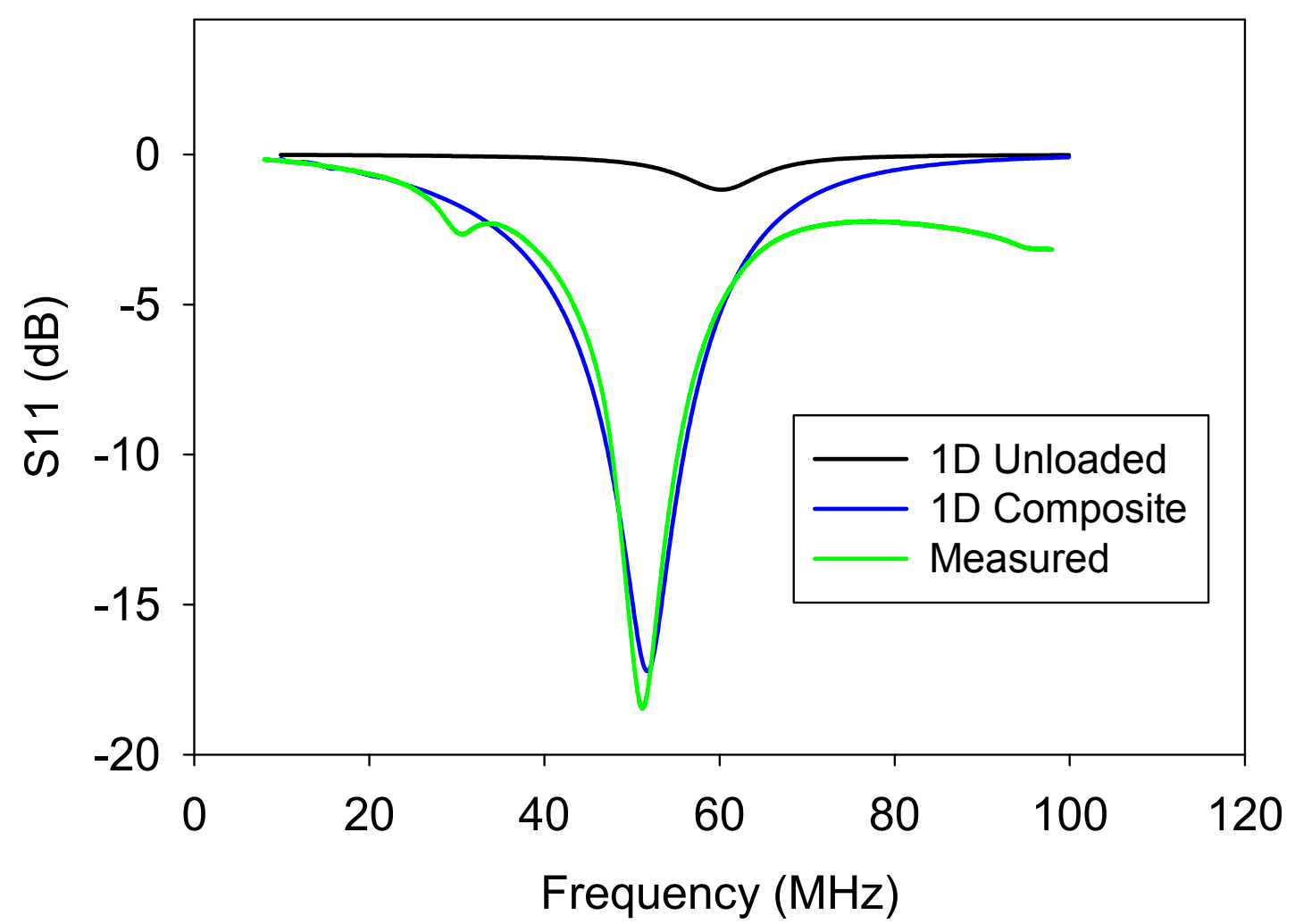

Figure 10 Return Loss (S11) for the unloaded and composite transducers based on 1D transmission line models. After proper layer-to-layer matching the return loss at the resonance frequency provided an excellent match to a $50 \mathrm{ohm}$ source. The return loss for the composite transducer was $-14.5 \mathrm{~dB}$ with a tunable range of $8 \mathrm{MHz}$ based on maintaining $90 \%$ input power coupling to the transducers.

The 1D model was used to compute the acoustic velocities at the faces of the composite transducer. For a P-P input voltage of $2.78 \mathrm{~V}$ the acoustic velocities at both faces was 3.4 $\mathrm{cm} / \mathrm{sec}$ at $54 \mathrm{MHz}$, which was computed with (0.35). The radiated acoustic power from the transducer was computed by applying $(0.36)$ and using the loaded impedances and velocities at the transducer faces. At $54 \mathrm{MHz}$ the total radiated acoustic power was 81.5 $\mathrm{mW}$.

\section{B. FEM Analysis of Bulk Acoustic Wave Transducer Array}

\section{Impedance of Transducer and Composite Structure}

To validate the physical model of the transducer using the FEM approach, the impedance of the transducer was calculated and used to extract the piezoelectric coupling coefficient $K$. To compute the impedance of the transducer a known voltage was applied to the driven electrode and then the induced surface charge was computed. By sweeping the frequency the impedance was calculated using (0.12). The piezoelectric coupling coefficient was computed from the known material parameters as defined in Table 1 and compared with the extracted $K$ from the FEM impedance data. The materials parameters gave $K_{33}=54 \%$ which agreed well with $K_{33(F E M)}=56.1 \%$ from 
the FEM model. This suggested that the 2D FEM model parameters are suitable for building more complex models of the acoustic transducer.

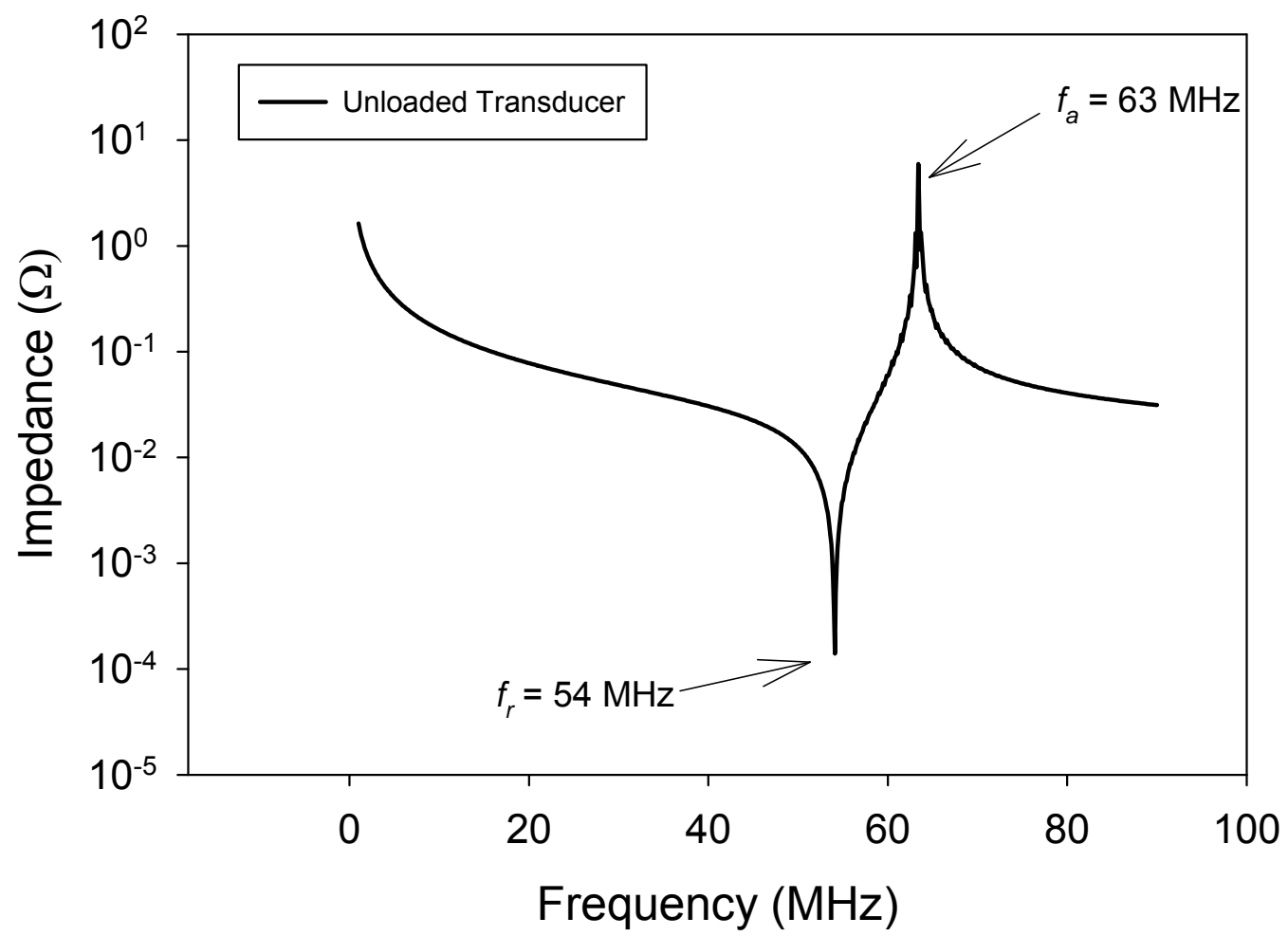

Figure 11 Extracted impedance response of 369 Y lithium niobate transducer from 2D FEM. The calculation was based on 2D FEM of the transducer with infinitesimally thin Au electrodes in air.

\section{Acoustic Pressure in Microchannel}

To understand the lysing mechanism with the microchannel, a 2D FEM model was used to compute the acoustic pressure distribution within the fluid region. This fluid region was modeled as a cross-section of the channel. Since the acoustic fields are nearly instantaneous compared to the mass transport through the microchannel, a 2D simulation was sufficient to capture the pressure field. In Figure 12a, the acoustic pressure is shown for an applied voltage of $3 \mathrm{~V}$ P-P across the transducer. The resulting peak acoustic field was 1 MPa with portions of the microchannel around $0.2 \mathrm{MPa}$. The acoustic pressure was computed along cross-sectional lines through the center of the microchannel both horizontally and vertical Figure $12 \mathrm{~b}$ and c. At $54 \mathrm{MHz}$, the acoustic pressure was maximized since the optimal coupling to the frequency source was matched. The BAW transducer had $90 \%$ electrical power coupling efficiency across a bandwidth of $8 \mathrm{MHz}$, which provided excellent operating range. The acoustic pressure profiles within the microchannel demonstrate the scale at which the pressure undergoes rapid changes which was on the order of $20 \mu \mathrm{m}$. 


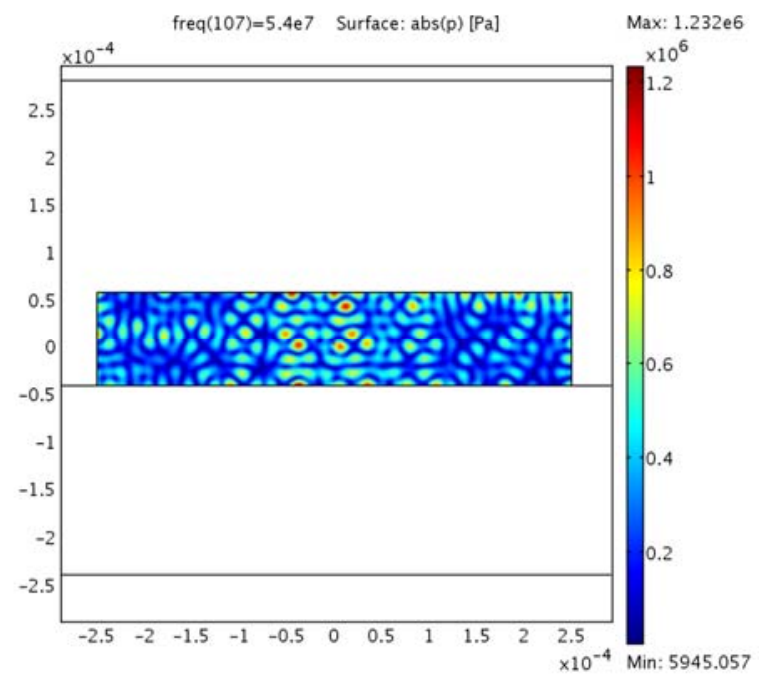

a)

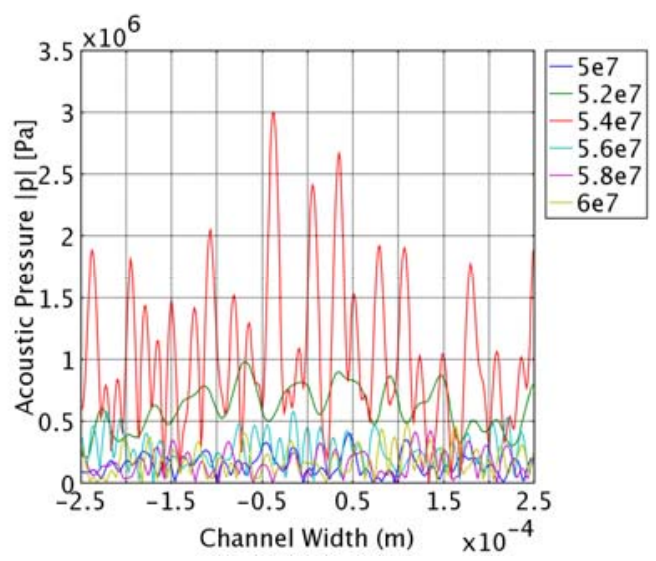

b)

Figure 12 Acoustic pressure within the microchannel cross-section. a) Acoustic pressure at 54MHz. The acoustic pressure was 1 MPa for an input of 3V P-P, b) Acoustic pressure at several drive frequencies across the width of the microchannel, c) Acoustic pressure at several drive frequencies across the height of the microchannel.

\section{Energy from Acoustic Fields in Microchannel}

In this computational effort, the energy of the acoustic fields was calculated versus the drive frequency. The motivation was to determine if other possible acoustic modes were leaking energy from the primary excitation frequency. Using (0.16), the kinetic and potential energy due to the pressure and velocity of the acoustic waves were computed versus the drive frequency. In Figure 13 the computational results indicate the primary BAW mode is being excited and converted to acoustic energy at $54 \mathrm{MHz}$. 


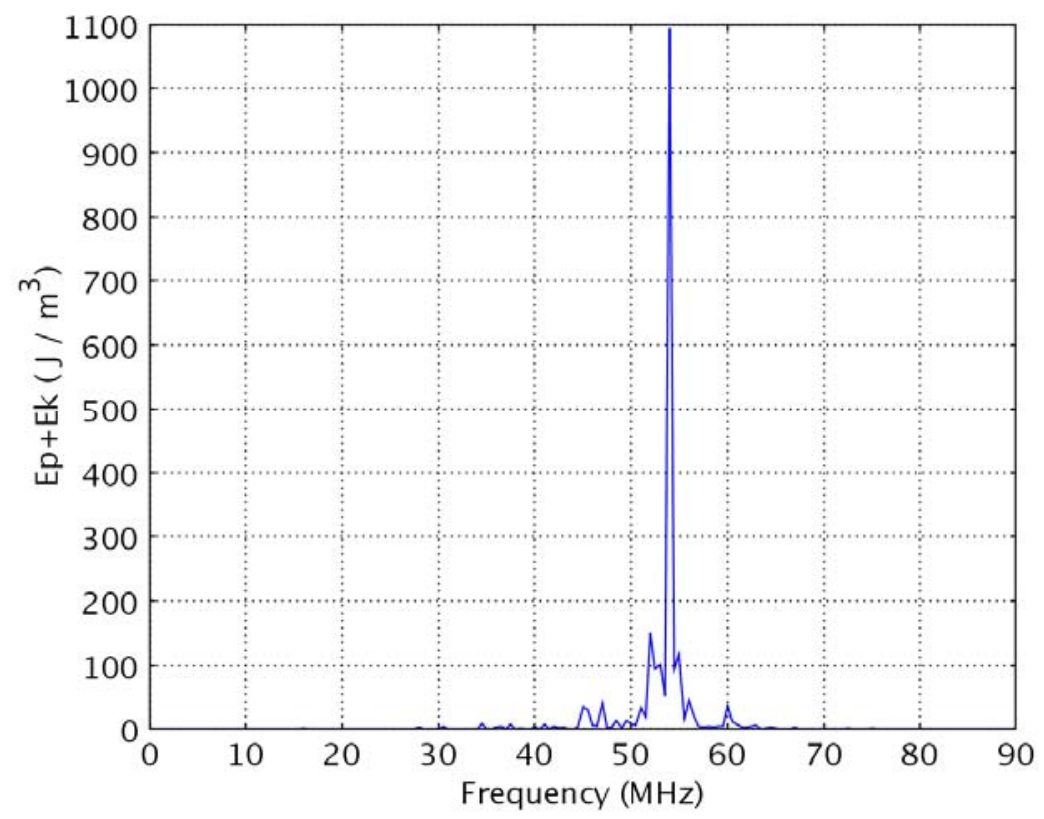

Figure 13 Kinetic and potential energy of the acoustic fields in the center of microchannel.

\section{Thermal Analysis}

The goal of this analysis was to compute the thermal variation from the inlet to the outlet of the microchannel. To perform this computation, the heat flux entering the boundary between the transducer and epoxy layers was required (Figure 8). This heat flux was the result of electrical and mechanical losses with the transducer. By using a heat flux discontinuities at these boundaries, the computation could be simplified from a time-dependent to steady state model. This was a valid assumption since the lysing time was sufficiently long (e.g. 20 seconds), nearly reach the steady state. First, the P-P voltage delivered to each BAW at $50 \Omega$ was computed $2.78 \mathrm{~V}-3.47 \mathrm{~V}$ which corresponded to the known input power (i.e. $21.9-23.8 \mathrm{dBm}$ ). Then the 1D transmission line model was used to compute the radiated power of the emitted acoustic waves. By knowing the applied power input, conservation of energy could be applied (0.37) to determine the mechanical and electrical loss. Since the transducer is most efficient at $52-54 \mathrm{MHz}$, the radiated acoustic power was taken at this frequency. These values were used in the 1D model to determine the radiated acoustic power, mechanical and electrical losses. Using $(0.36)$ and $(0.37)$ the combined loss from the mechanical and electrical processes were $11.1 \mathrm{~mW}$ with a radiated acoustic power of $81.5 \mathrm{~mW}$. The electrical loss $\left(P_{e}\right)$ was only $0.7 \mathrm{~mW}$. The power density across the transducer was $1570 \mathrm{~W} / \mathrm{m}^{2}$ for a delivered power input of $21.9 \mathrm{dBm}$.

Table 2 Electrical Power delivered to BAW Transducers and Resultant Input Power Density

\begin{tabular}{|c|c|c|c|c|}
\hline $\begin{array}{c}\text { Delivered } \\
\text { Power }^{\mathbf{1}} \\
(\mathbf{d B m})\end{array}$ & $\begin{array}{c}\text { Equivalent } \\
\text { Power } \\
(\mathbf{m W})\end{array}$ & $\begin{array}{c}\text { Induced P-P } \\
\text { Voltage for 50 } \\
\mathbf{\Omega} \text { load } \\
\text { (V) }\end{array}$ & $\begin{array}{c}\mathbf{P}_{\mathbf{e}}+\mathbf{P}_{\mathbf{m}} \\
\mathbf{( \mathbf { m W } )}\end{array}$ & $\begin{array}{c}\text { Power } \\
\text { Density } \\
\text { due to losses } \\
\left(\mathbf{W} / \mathbf{m}^{\mathbf{2}}\right)\end{array}$ \\
\hline 21.9 & 154.4 & 2.78 & 11.1 & 1570.3 \\
\hline 22.8 & 192.9 & 3.11 & 13.9 & 1966.4 \\
\hline 23.8 & 241.1 & 3.47 & 17.3 & 2447.4 \\
\hline
\end{tabular}


${ }^{1}$ Delivered power is the electrical power received by the transducer as if it was perfectly matched. This 3D model captured the physical boundary conditions and operation of the actual device (Figure 1). To represent the operation of the BAW acoustic transducers, the interface between the acoustic BAW transducers and the conductive epoxy were modeled as heat flux discontinuities. This model was solved for the steady state condition. At this boundary heat enters the system from acoustic mechanical and electrical losses $\left(P_{m}+P_{e}=1570 \mathrm{~W} / \mathrm{m}^{2}\right)$. This was a reasonable simplification since the heat source is strictly a result of the losses in the system and eliminates the need to compute time dependent problems.

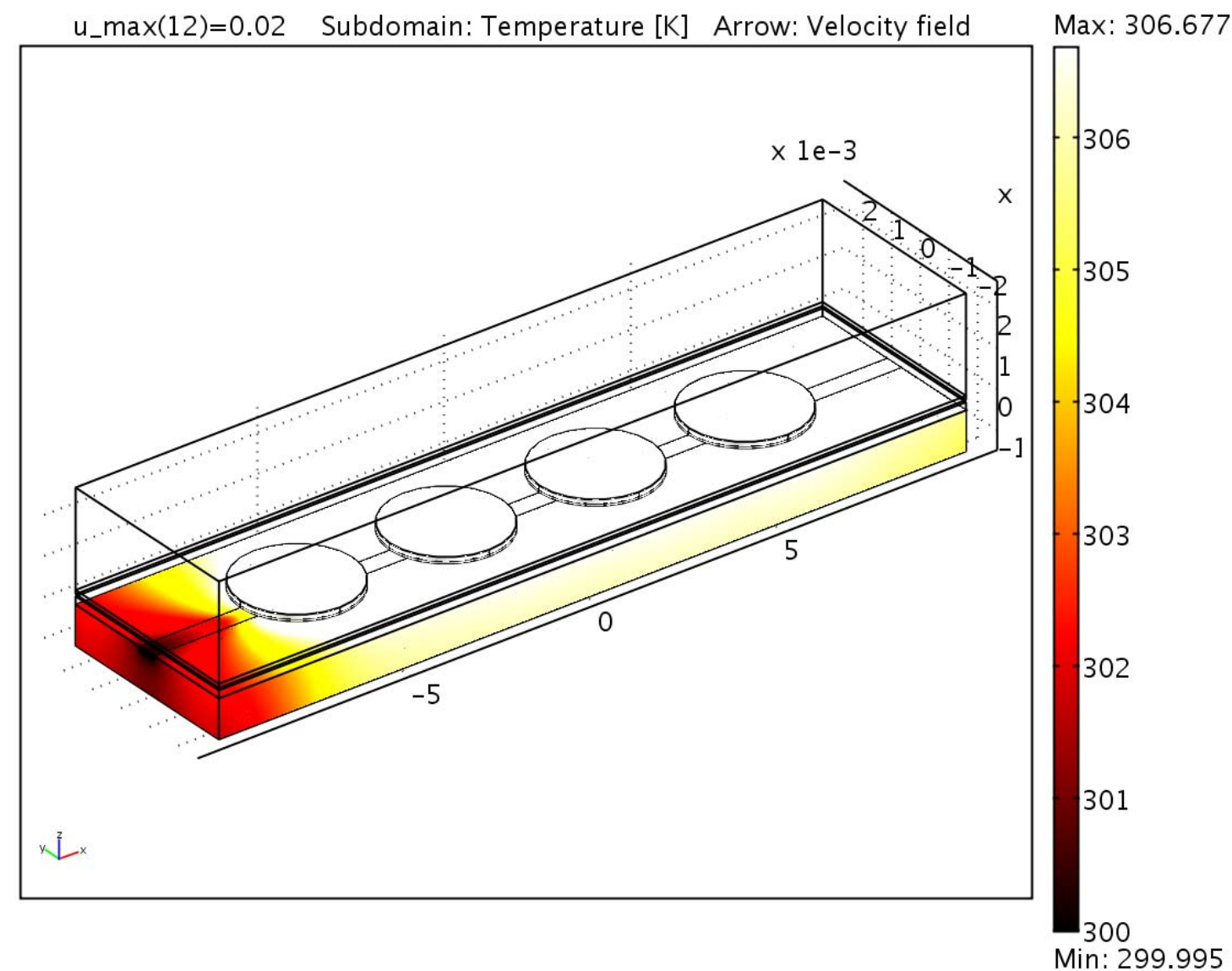

Figure 14 3D Thermal model of BAW transducer array. This analysis was done at $u_{\max }=0.02 \mathrm{~m} / \mathrm{s}$ which is 60 $\mu \mathrm{l} / \mathrm{min}$. The channel inlet (lower left) starts at $300 \mathrm{~K}$, reaching a steady state value of $306 \mathrm{~K}$ at the outlet (upper right). The input power to each transducer was $21.9 \mathrm{dBm}$.

At a power input of $23.8 \mathrm{dBm}$, significant heating was observed at the microchannel outlet for the lower flow rates (i.e. $5-30 \mathrm{\mu l} / \mathrm{min}$ ). Our lysing results indicated that a power input of $21.9 \mathrm{dBm}$ was more than sufficient to disrupt the cellular membranes at a flow rate of $40-50 \mathrm{\mu l} / \mathrm{min}$. In the experimental lysing studies, the steady state condition was never quite reached as indicated by thermal measurements taken at the transducer. The thermal results in Figure 15 are for the steady state condition and therefore are slightly higher than for an actual lysing event. The experimentally measured temperatures were slightly less than the model due to thermal separation between the RTD sensor and the actual outlet. 


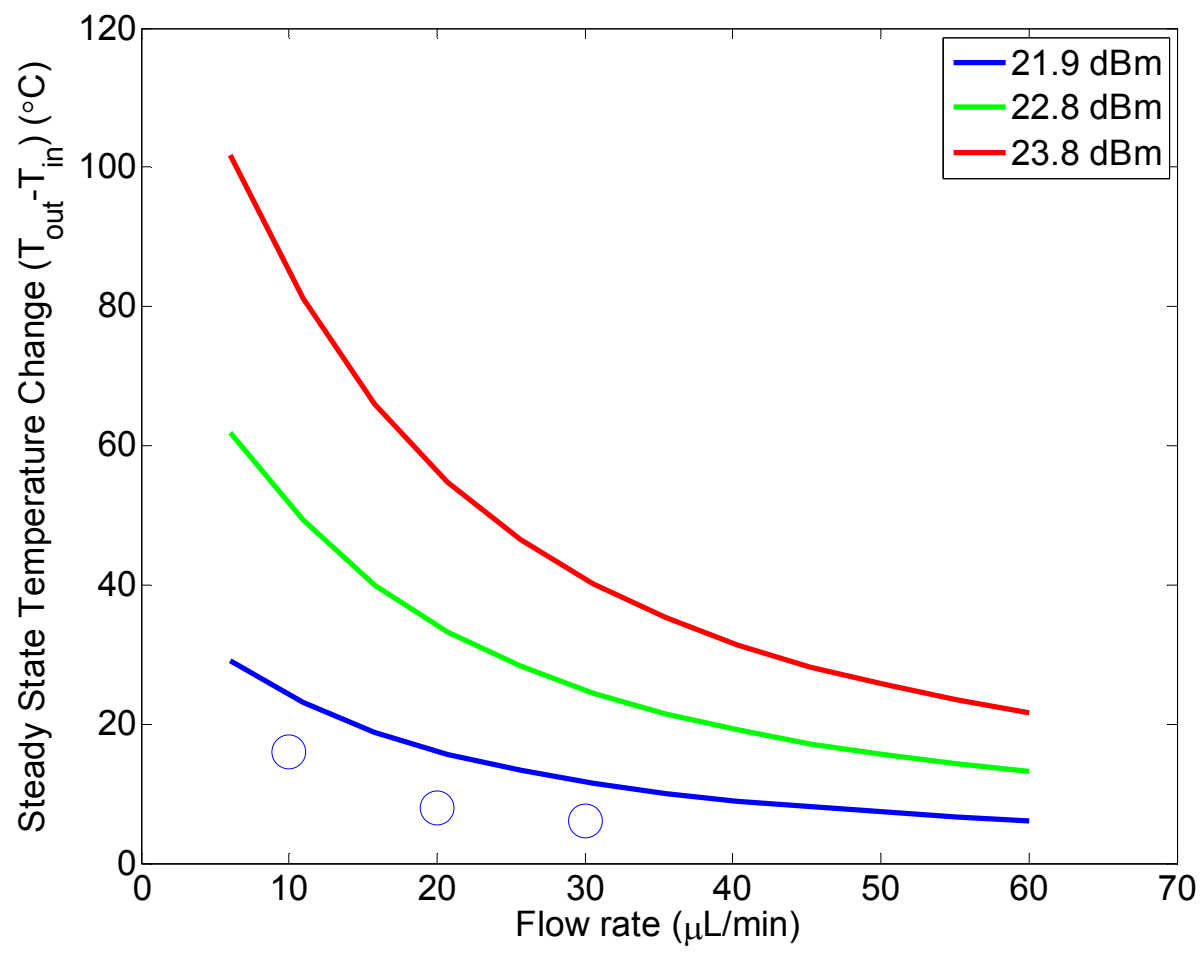

Figure 15 Steady state thermal response versus the input power to the BAW lysing transducer. The open blue circles are the experimentally measured temperature near the microchannel outlet.

\section{Cavitation Threshold}

Reducing the effects of cavitation is critical to ensure optimal lysing efficiency within a microchannel format. Though cavitation (i.e. rapid formation and collapse of bubbles within a fluid) may cause cell lysis, the formation of air bubbles within the microchannels dramatically reduces the flow rate. Previous calculations indicate that cavitation is frequency dependent with a threshold of $\sim 8 \cdot 10^{5} \mathrm{~W} / \mathrm{m}^{2}$ at $50 \mathrm{MHz}$ [16]. At a power density above this threshold cavitation occurs in the fluid. To compare with the actual device the cross-sectional area of the transducer was $\pi r^{2}=\pi(1.5 \mathrm{~mm})^{2}=7.1 \cdot 10^{-6}$ $\mathrm{m}^{2}$. The power applied to each transducer was $\approx 143 \mathrm{~mW}$, giving a total of $8.1 \cdot 10^{4}$ $\mathrm{W} / \mathrm{m}^{2}$, indicating operation was below the cavitation threshold. Cavitation was observed at the highest power setting of $23.8 \mathrm{dBm}$ which corresponded to $241.1 \mathrm{~mW}$ delivered to each transducer or $1.3 \cdot 10^{5} \mathrm{~W} / \mathrm{m}^{2}$. This value was on the order of the reported cavitation threshold.

\section{RF Drive Circuit}

The RF drive circuit consisted of a tunable RF source ranging from 48 to $58 \mathrm{MHz}$ connected to a RF power amplifier (Figure 16). Frequency tuning was needed to account for changes in the fabrication process due to variations in transducer mass loading and bonding. The RF source was amplified using a 2W fixed amplifer ZHL-1-2W$S$ (Minicircuits, Brooklyn, NY). The power input was configured at deliver a maximum of 
$200 \mathrm{~mW}$ to each transducer. Previous experiments were performed to set an upper limit on the maximum power delivered to transducer thereby minimizing cavitation.

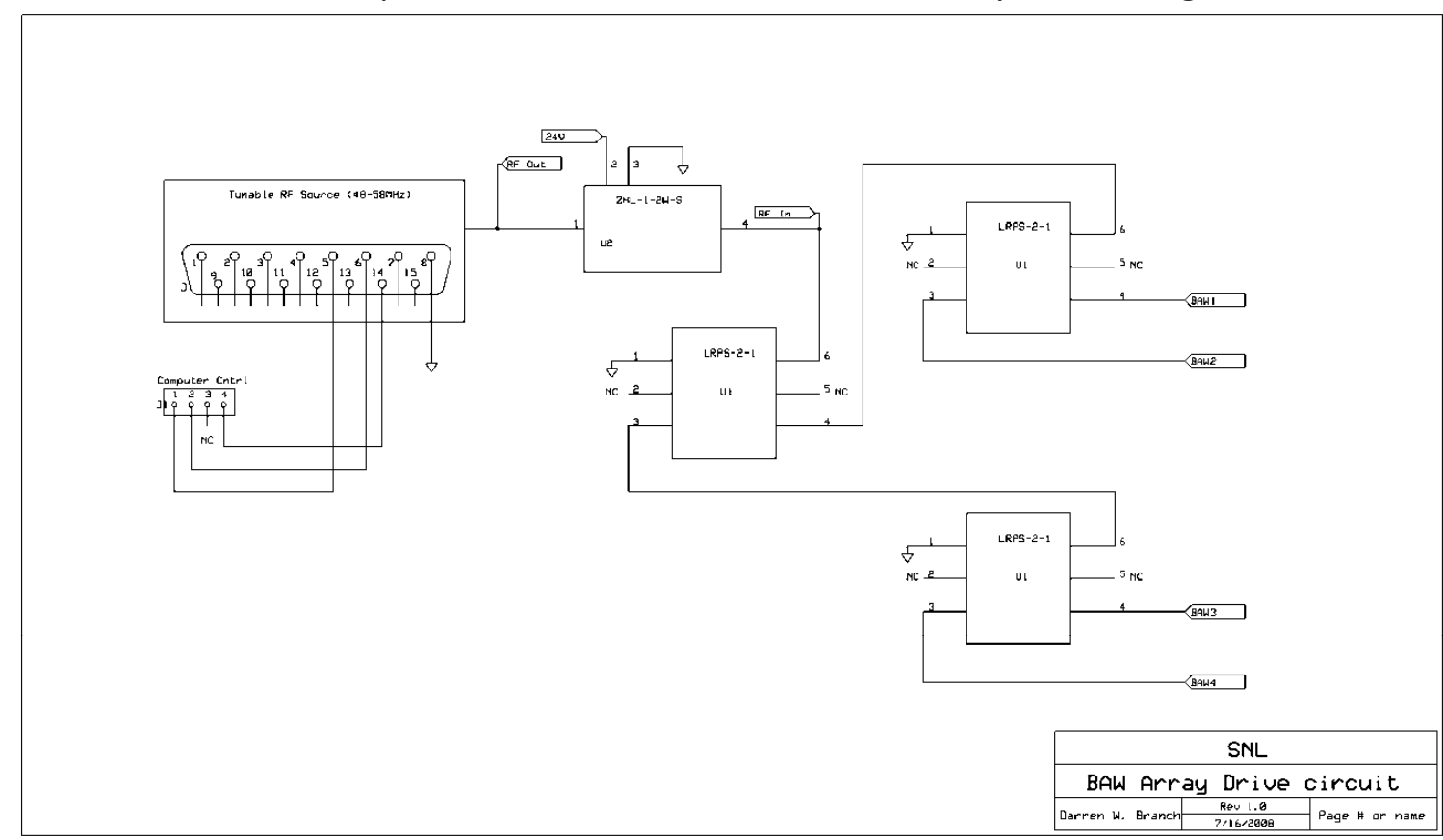

Figure 16 Schematic of RF drive circuit for BAW lysing system. Computer control was used to tune the frequency of the RF source (output power $-0 \mathrm{dBm}$ ) which was then connected to a $2 \mathrm{~W}$ RF power amplifier. This was split 4 ways to drive each BAW transducer.

\section{BAW Lysing System}

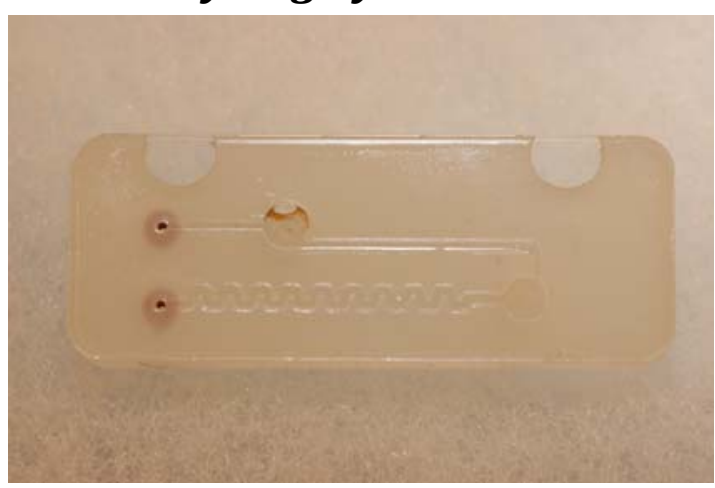

a)

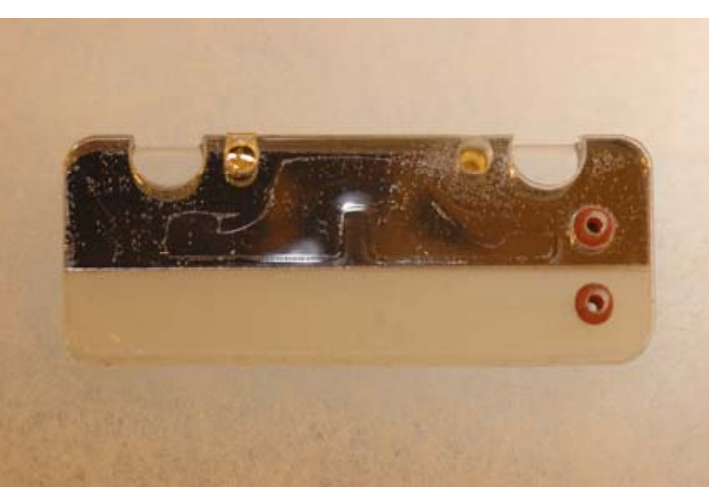

b) 


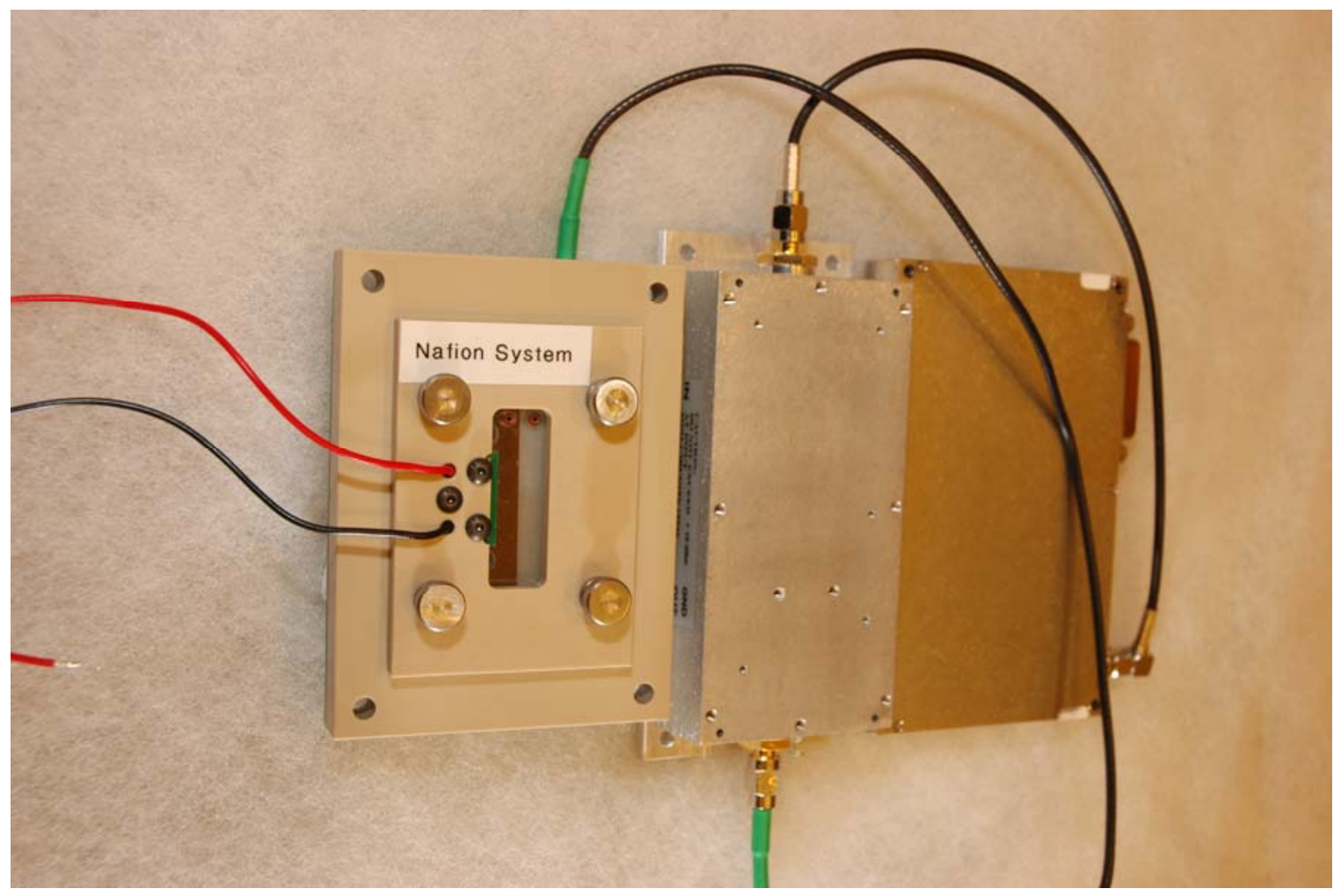

c)

Figure 17 BAW lysing components. a) Nucleic acid binding magnetic bead based cartridge, b). Nafion coated gold electrodes reversibly bind nucleic acid and c). Complete lysing/nucleic acid extraction system.

\section{Key Electrical Components}

- Tunable RF Source (48-58MHz) (Praxsym, Fisher, IL)

- 2 W Power Amplifier ZHL-1-2W-S (Minicircuits, Brooklyn, NY).

- Five element BAW array, $52 \mathrm{MHz}$ with $8 \mathrm{MHz}$ bandwidth

- Power splitters (LRPS-2-1) $1 \mathrm{~W}$ (Minicircuits, Brooklyn, NY).

\section{BAW System Operation}

\section{a. BAW Device Performance}

The scattering parameter S11 was used to characterize the electrical performance of the BAW transducer prior to assembly with the microfluidic cartridge. S11 is a complexvalued quantity that is equivalent to the measured reflection coefficient, and from which the power delivered to the device could be calculated. S11 was recorded across a $10 \mathrm{MHz}-100 \mathrm{MHz}$ band using an 8358A network analyzer (Agilent, Santa Clara, CA) in accordance with the manufacturer's specifications prior to each experiment. The power delivered to the device was chosen to maximize lysing efficiency without causing cavitation or excessive heating of the transducer and heat sink. 


\section{b. BAW Array Lysing Efficacy}

The results of the experiments comparing the lysing efficacy of the microfluidic lysis platforms with the commercial ultrasonication device are presented in Figure 18a. The graph demonstrates that the lysing efficacy of BAW microfluidic lysing system is very competitive with the commercial system, requiring much lower power input (i.e. $\sim 200$ $\mathrm{mW}$ as compared to the commercial system operating at $12-58.5 \mathrm{~W}$ ). The performance gain of the microfluidic lysing system was attributed to the operating frequency and hence the wavelength of the propagating acoustic waves in the fluid. For maximum transfer of acoustic energy to the biological cells, the acoustic wavelength, $\lambda$, should approach the size of the cell, which in the case of $E$. coli, is $\sim 2 \mu \mathrm{m}$. Given the speed of sound, $v$, in water is $\sim 1500 \mathrm{~m} / \mathrm{s}$, the relationship $\lambda=v / f$ can be used to predict the wavelength of the acoustic pressure wave for the commercial and BAW devices. At an operating frequency, $f$, of $20 \mathrm{kHz}$, the acoustic wavelength of the commercial transducer was $75 \mathrm{~mm}$, while the wavelength of the $54 \mathrm{MHz}$ BAW array was $27.8 \mu \mathrm{m}$, allowing for efficient energy coupling to the cells. The error bars at each data point indicate that for some experiments, there was large variation in viable cell counts. This effect was caused by the number of serial dilutions that were required to obtain appropriate plate counts, resulting in a $10^{5}$ dilution factor that amplified small variations in counting. ATP release as a function of the acoustic lysis method is shown in Figure 18. The commercial acoustic finger liberated substantially more ATP from treated samples than the BAW microfluidic lysing system, however with only a $15 \%$ increase in cell lysis (Figure 18a). We suspect that the large amount of ATP release was due to a sonoporation driven process where cell membrane releases ATP without complete disruption of the membrane. Our results show that the ATP release was not indicative of improved cell lysis given the marginal difference in \% of cells lysed from the plating assay. This strongly suggests that the acoustic finger allows cells to maintain viability even after exposure for 20 minutes. 


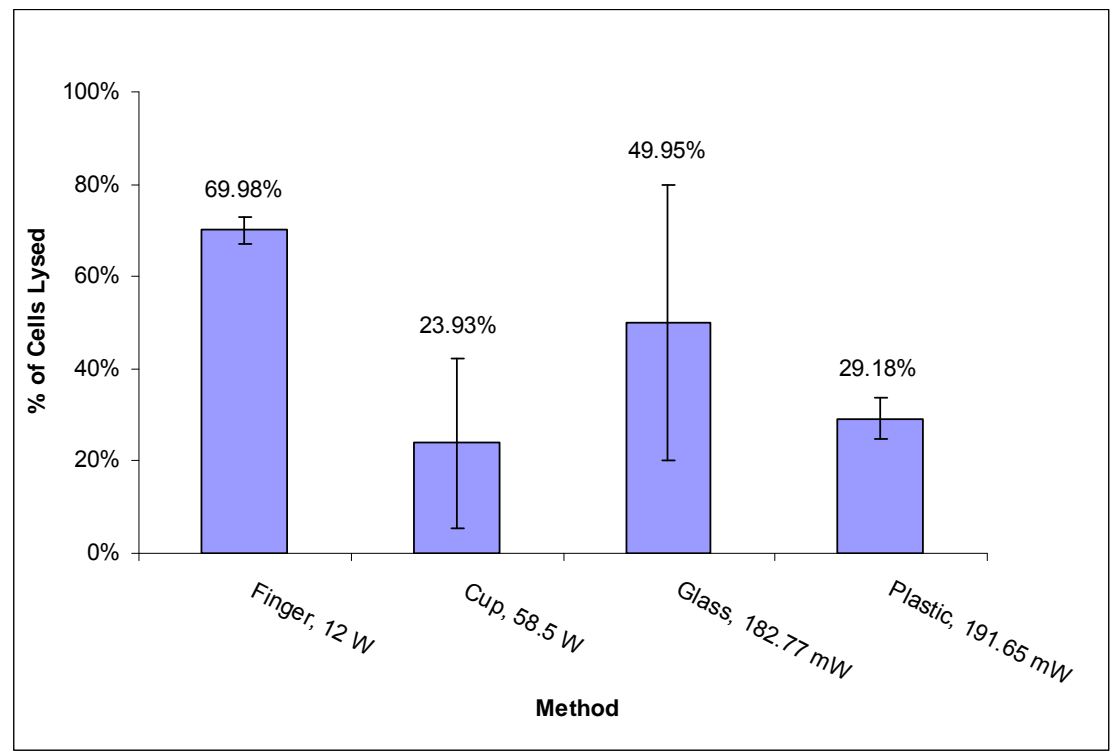

a)

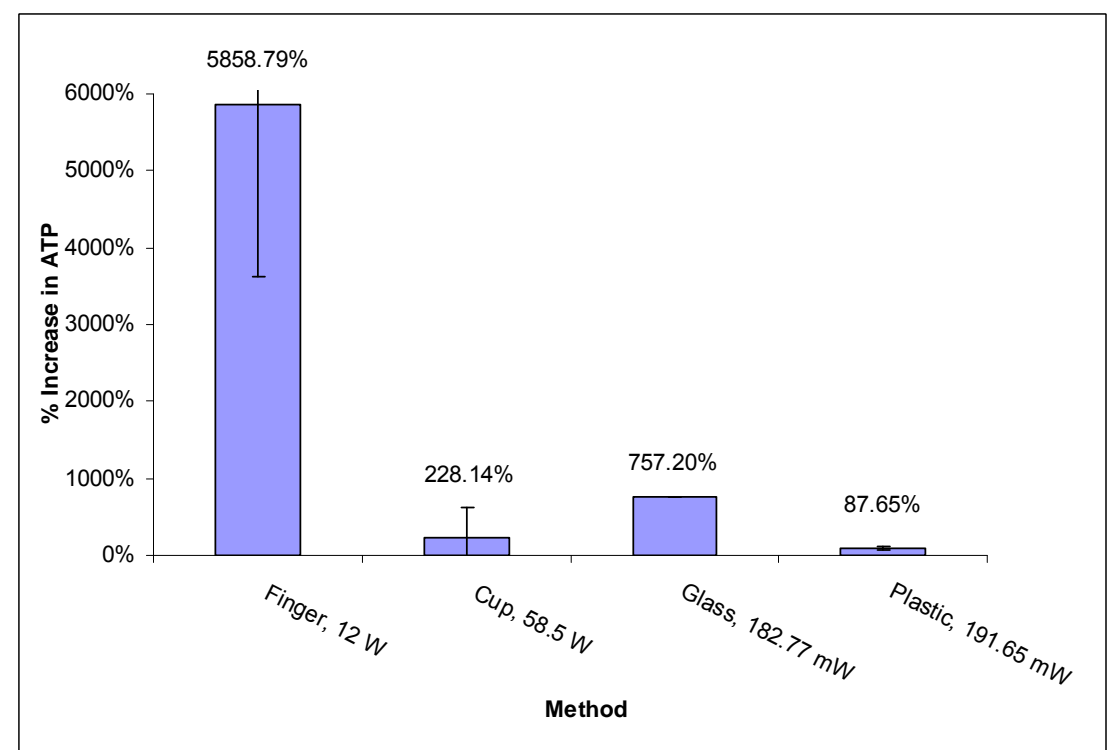

b)

Figure 18 Comparison of cell lysis efficacy for commercial and microacoustic methods. a) Cell culture plating viability assay, b) ATP assay to determine cell lysis. 'Glass' and 'Plastic' refer to the coupling layer between the transducer and microchannel. It was expected that glass would be less acoustically lossy than plastic and hence improve cell lysis. The data for the two microfluidic systems were measured at $10 \mu \mathrm{L} / \mathrm{min}$ flow rates. $\mathrm{N}=10$.

Figure 19a and b compare the performance of the BAW microfluidic lysing system with either a glass or plastic coupling layer as a function of power delivered to the transducer. Cell lysis efficacy was 5-20\% higher for microfluidic cartridges with glass bases as compared to ones with plastic bases. This result was expected since glass has very low acoustic loss as compared to plastic. However, this result demonstrates that ability to lyse cells using a completely plastic based microchannel, which substantially simplifies fabrication complexity and cost. 
Acoustic losses and flow rate in the channel were compensated using a serpentine channel to increase the exposure time to the acoustic pressure field. The additional path length provided an additional tuning parameter between lysing efficacy and the subsequent nucleic acid extraction. For both systems, Figure 19 and b demonstrate that increased power improved lysing efficiency, which required a balance between input power and heat generation by the transducer according the thermal analysis (Figure 14). 


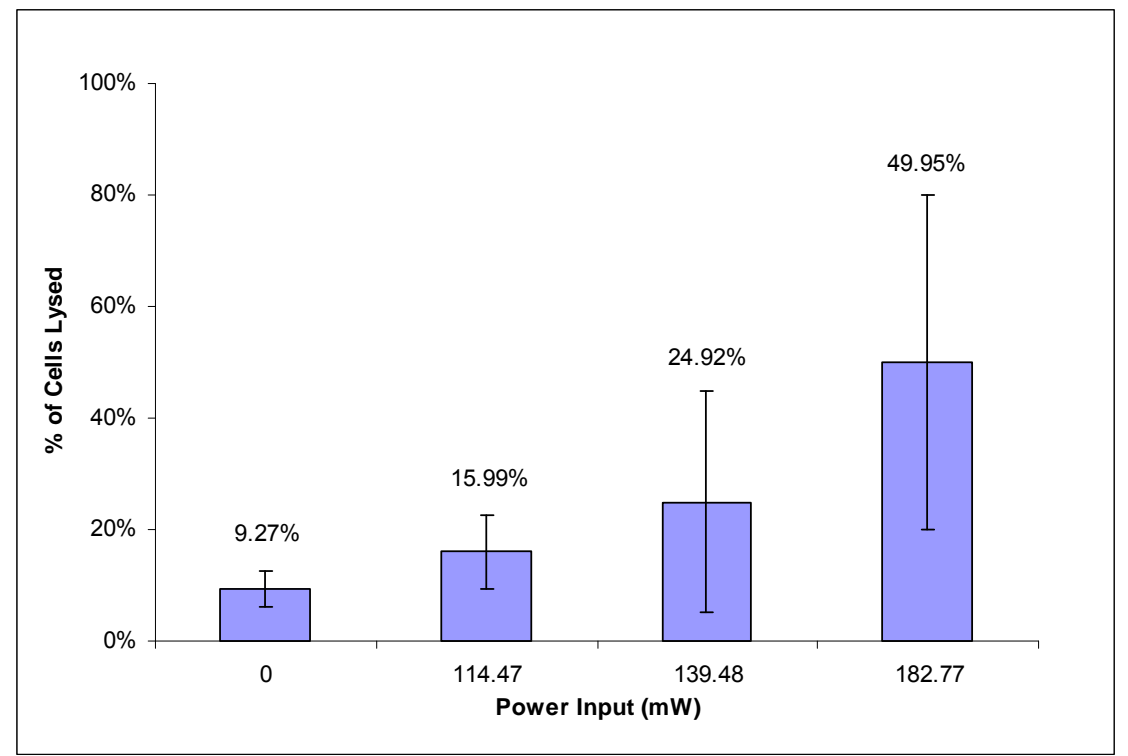

a)

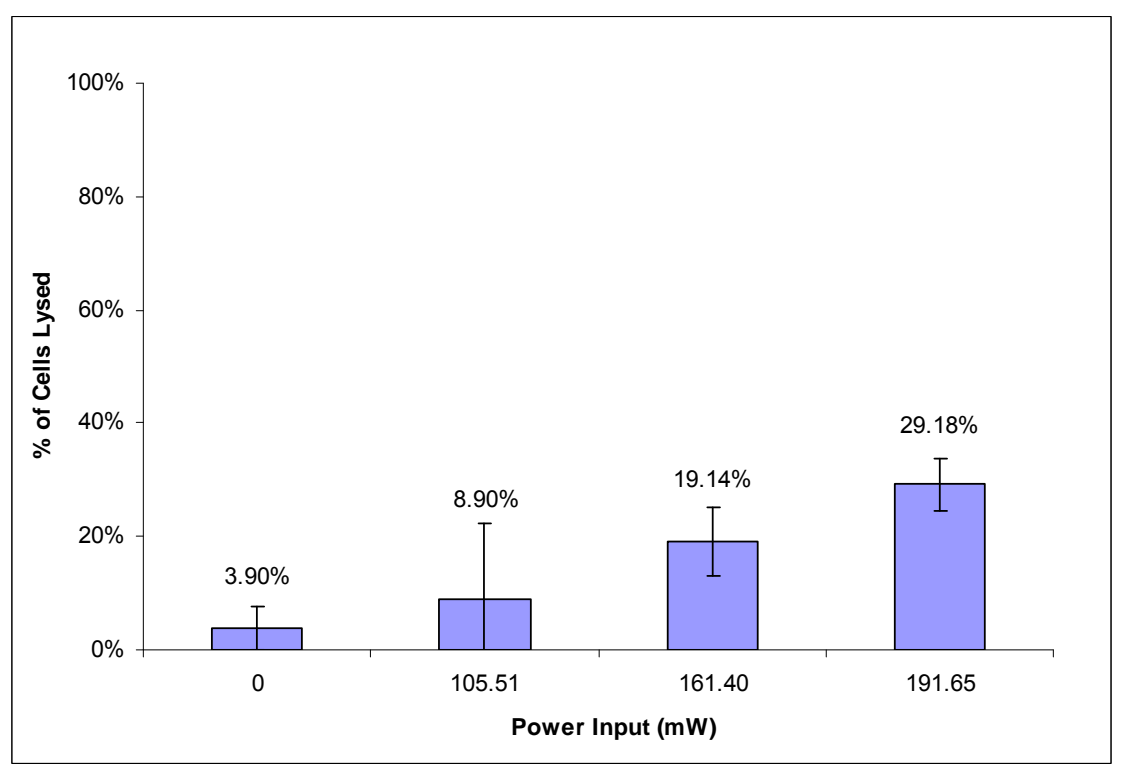

b)

Figure 19 Cell lysis efficacy the BAW transducer array versus input power, a) Glass base, b) Plastic base. The flow rate was $10 \mu \mathrm{L} / \mathrm{min}$. $\mathrm{N}=10$.

\section{E. Nucleic Acid Extraction}

\section{Sol-gel Packed Microchannels}

The first prototype DNA extraction microchambers were fabricated at an external supplier (Micronit Microfluidics) using wet etching of borosilicate glass substrates. Two standard designs have been fabricated: (1) $50 \mu \mathrm{m}$ wide, $20 \mu \mathrm{m}$ deep, and $332 \mathrm{~mm}$ length channels, and (2) $150 \mu \mathrm{m}$ wide, $150 \mu \mathrm{m}$ deep, and $332 \mathrm{~mm}$ length channels 
(Figure 20a). The first design contained a coverslip, which allowed for high resolution confocal imaging for troubleshooting device operation. The second design was more readily compatible with processing higher sample volumes due to the deeper channel and increased surface area.

We pursued two paths for integrating the sol-gel silica bead matrix into microfluidic chambers: injection into closed chambers and aerosol spraying into open chambers (Figure 20b).

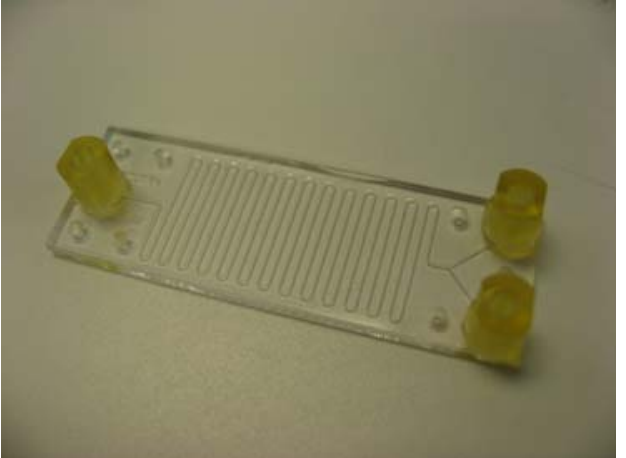

a)

Figure 20 Sol-gel silica bead matrix extraction cartridge. a) Prototype DNA extraction microchamber. Chip dimensions were $45 \times 15 \times 1.25 \mathrm{~mm}$. b) Injection and spray methods for integrating the DNA extraction matrix into the microchamber.

The injection method was used with closed microchambers, and the sol-gel-bead matrix was injected into the channel under pressure (or vacuum). One end was plugged to achieve high density packing, and then the sol-gel was cured at an elevated temperature to lock beads in place. However, it was difficult to achieve reliable high density packing using this method, and the high pressures (or vacuum) required for packing can lead to device failure. Figure 21 shows a closed microchamber with $150 \mu \mathrm{m}$ wide channels that was filled with the sol-gel-bead matrix.

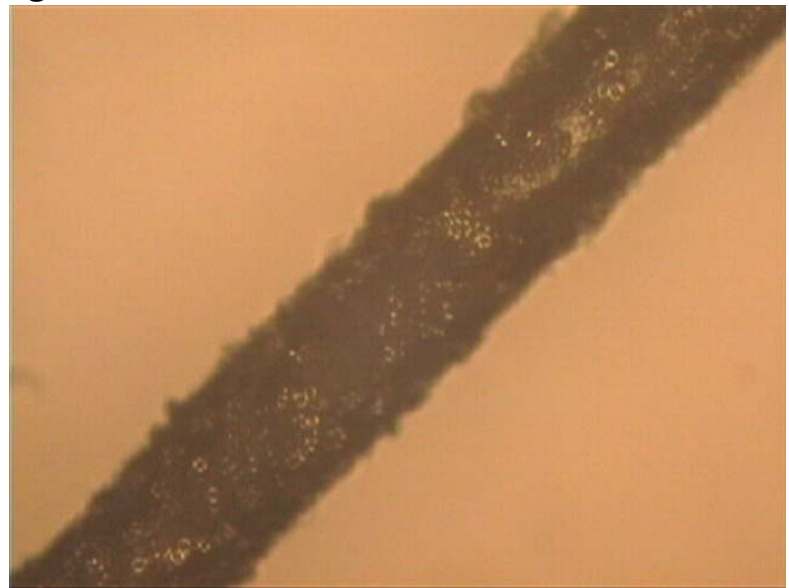

Figure 21 Sol-gel-bead matrix cured within a closed microchamber.

Tetraethyl orthosilicate (TEOS) based sol-gel and beads ( $15 \mu \mathrm{m}$ diameter silica) were mixed at a concentration of $200 \mathrm{mg}$ of beads in $1 \mathrm{ml}$ of sol-gel. The chamber outlet was plugged $\sim 90 \%$ to allow some fluid to exit, but confine a substantial amount of beads in the channel. After filling, the chamber was dried at $120^{\circ} \mathrm{C}$ for 24 hours. The device 
shown in Figure 21 had several regions that were completely packed with matrix, but in general the coverage and packing was less than optimal.

The second matrix integration method utilized an aerosol spray apparatus to incorporate the DNA extraction matrix into microchambers. The aerosol spray method has been used to produce reliable, high surface area sol-gel matrices, with controllable pore sizes. The high surface area is advantageous for maximizing DNA extraction from a sample, but implementing the spray method with microchambers presented several obstacles. The microchamber must be open, and a mask must be used to confine the matrix to the interior of the microchamber and keep the top surface of the chip clean and debris free for subsequent bonding of the chamber lid (Figure 20, far right). The best strategy discovered was to mask off the base substrate, spray the matrix, and then remove the patterning mask. Figure 22 shows an example of the spraying and masking method. We used a laser-cut adhesive to serve as the mask for a silicon substrate capped with a thermal oxide. Several passes of the aerosol spray were used to coat the substrate, and then the mask was mechanically removed.
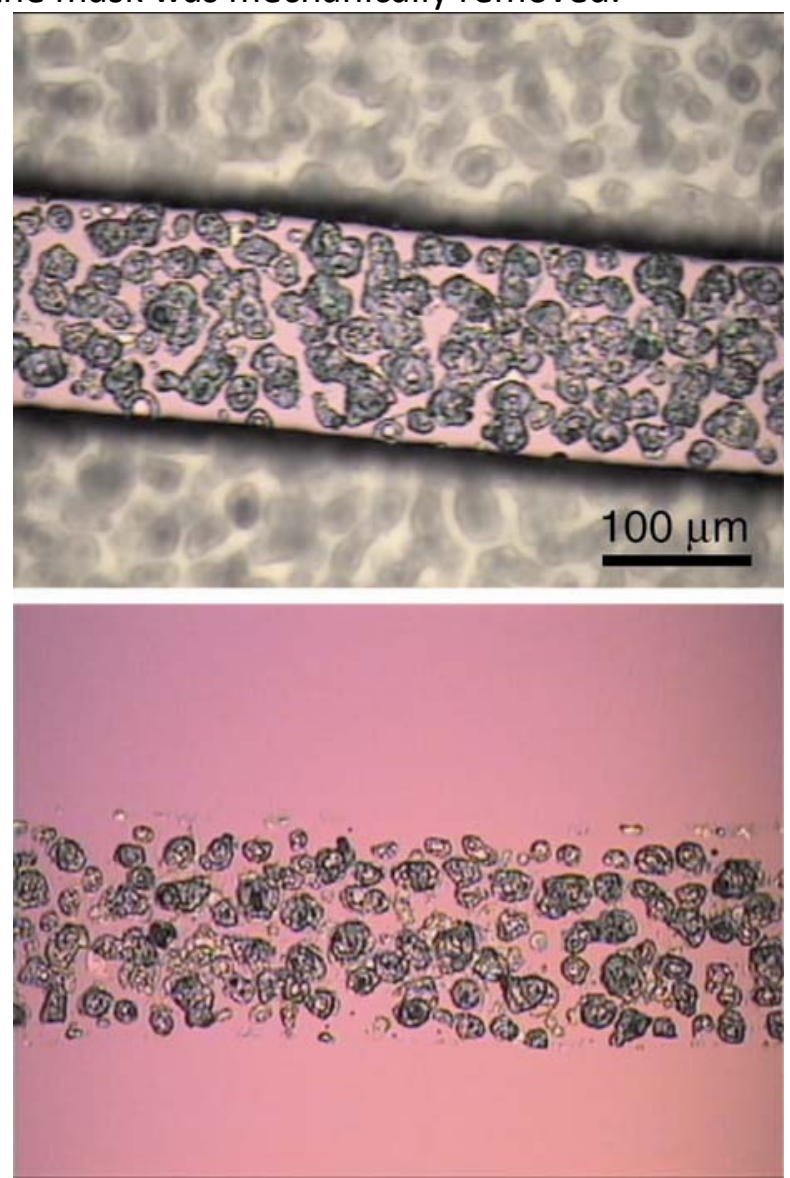

Figure 22 Patterning of DNA extraction matrix (top). After removal of patterning mask (bottom). In our final implementation we successfully developed a method to integrate a DNA extraction matrix into glass microchambers. Sol-gel and silica bead matrices bind DNA/RNA to their matrices through an electrostatic interaction. The silica beads provide a large surface area for selective binding of DNA, while the sol-gel serves as a silica-based glue to hold beads in place during device operation. An injection method 
was used to load the sol-gel silica bead matrix into the microfluidic channels by plugging the end of the channel (Figure 23a). The packed bed length was $10-20 \mathrm{~mm}$ in length and the channel dimensions were $150 \mu \mathrm{m}$ wide, $150 \mu \mathrm{m}$ deep, and $332 \mu \mathrm{m}$ in length (Figure 23b). Tetraethyl orthosilicate (TEOS) based sol-gel and beads ( $15 \mu \mathrm{m}$ diameter silica) were mixed at a concentration of $200 \mathrm{mg}$ of beads in $1 \mathrm{ml}$ of sol-gel. The chamber outlet was plugged $\sim 90 \%$ to allow some fluid to exit, confining a substantial amount of beads in the channel. After filling the chamber, the chamber was dried at $120^{\circ} \mathrm{C}$ for 24 hours. The device shown in Figure $23 \mathrm{~b}$ had several regions that were completely packed with matrix, but in general the coverage and packing was slightly less than optimal. Using the system in Figure 23b, samples of DNA were extracted from a sample solution containing $250 \mathrm{fmol}$ of DNA. The DNA bound to the silica matrix, allowed excess lysate (e.g. proteins) to pass through as waste. Fractions were collected during the entire process to isolate the DNA from excess lysate as shown in Figure $23 \mathrm{~d}$. The extraction efficiency was $40 \%$.

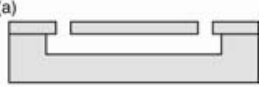

(b)

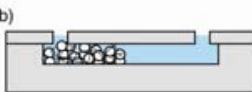

a)

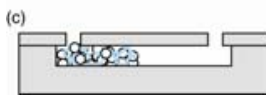

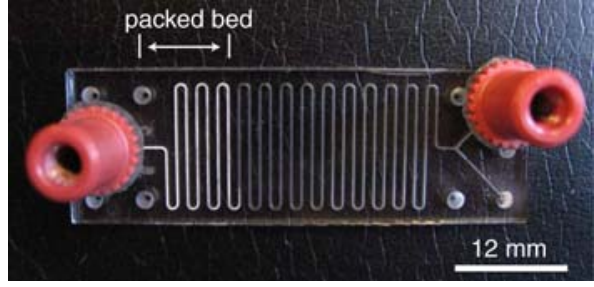

b)

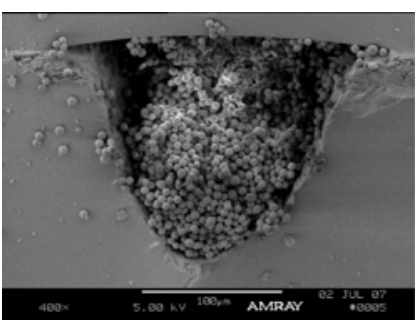

C)

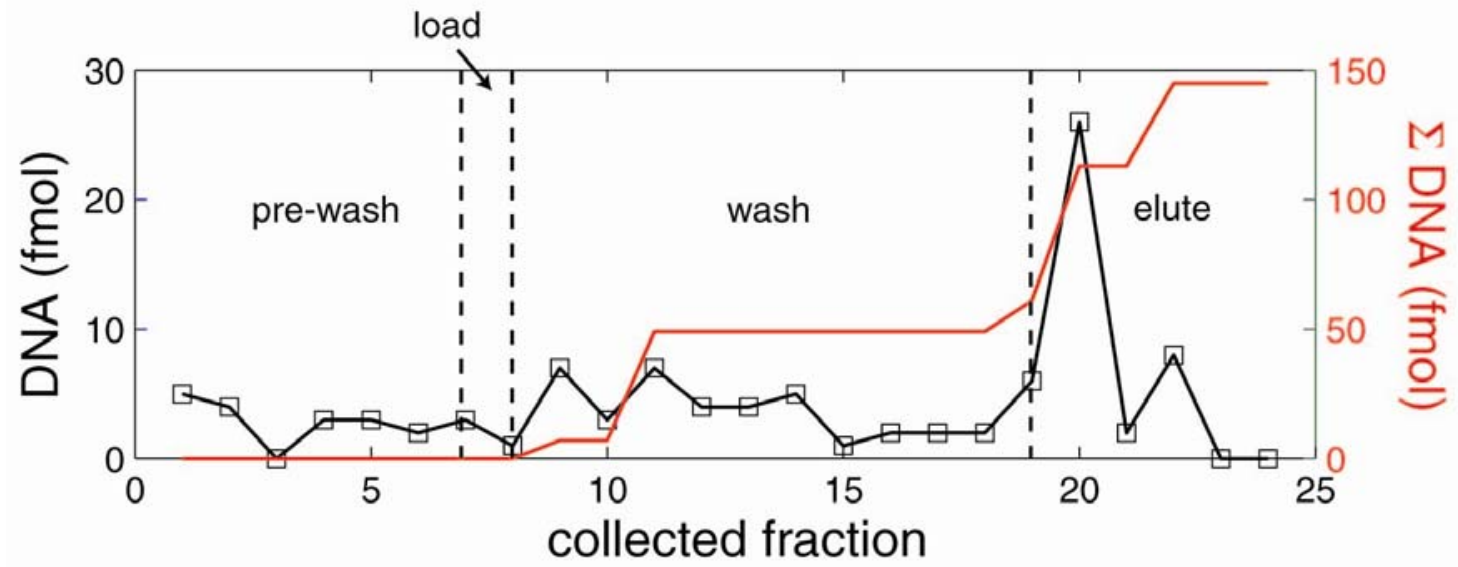

d)

Figure 23 DNA extraction using a silica-bead matrix. a) Packed microchannel, b) Actual DNA extraction device, c) End of the packed bed section of sol-gel immobilized silica beads $(5 \mu \mathrm{m})$ in channel, d) Extraction profile (black) and cumulative extraction (red) of DNA from a packed bed device as a function of collected fraction. The extraction efficiency was $40 \%$.

\section{Magnetic Beads}

For each of the three cartridge designs tested, approximately $1 \times 10^{6}$ beads were loaded into the channel for DNA extraction. This number was verified using flow cytometry of the bead solution by comparing the particle counts prior to loading with the eluent 
collected following loading of the beads into the channel. Quantities greater than $1 x$ $10^{6}$ beads tended to block fluid flow in the channel, and sufficient pressures could not be generated to push the fluid through the device without compromising the integrity of the cartridge.

Figure 24 compares the DNA extraction performance of the ChargeSwitch ${ }^{\circledR}$ beads using channel designs 1 and 2 when 201 ng of DNA was loaded into the channel. Cartridge design 1 displays superior performance with 21.93 ng DNA recovered in elution vs. 8.63 ng recovered using cartridge design 2 . DNA also appears to be more efficiently bound during the binding step with less non-specifically bound DNA being removed during the wash step. One significant difference between these two channels was the size of the magnet used, and hence, the size of the bead reservoir. The magnetic field produced by the $1 / 4$ " diameter magnet was large enough to trap beads well outside the bead reservoir and all along the serpentine channel used for cell lysis (Figure 5a for channel 1 design). This may have increased the surface area of the beads available for binding of DNA, compared to channel design 2 , in which the beads were more tightly packed in a smaller bead reservoir over a 1/8" diameter magnet (Figure $5 b$ for channel 2 design). However, for design 2, the bead reservoir was situated over an acoustic transducer, which was designed to facilitate mixing of the beads and streaming of the DNA from the bead surface during the elution step. The $54 \mathrm{MHz}$ acoustic transducer was driven at 136 $\mathrm{mW}$, with a $25 \%$ duty cycle. From Figure 24 , it appears that the most significant improvement to this assay using design 2 could be made in the binding step. These experiments did not involve activation of the acoustic transducer during the binding step, which may have improved mixing of the beads with the DNA solution and enhanced DNA binding.

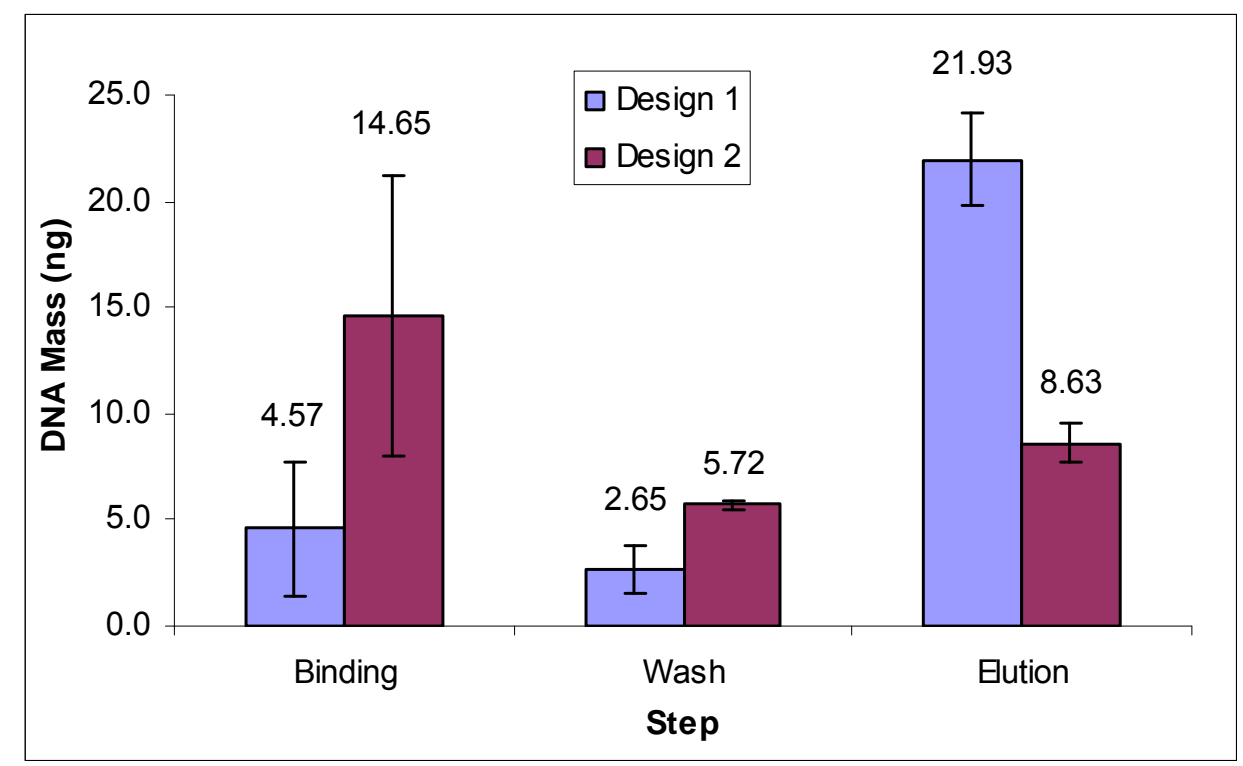

Figure 24 Mass of DNA extracted using ChargeSwitch ${ }^{\circledR}$ beads with microfluidic cartridge designs 1 and 2 and a sample flow rate of $10 \mu \mathrm{L} / \mathrm{min}$.

Figure 25 presents data from microfluidic cartridge design 3. This cartridge design was similar to design 2, but incorporated a larger bead reservoir in layers 3-6 (openings are 
not shown in Figure $5 b$ ) to provide increased volume for mixing of the beads with DNA when driven acoustically (Figure $5 b$ for channel 3 design). Furthermore, the assay time was significantly decreased by increasing the flow rate for all steps to $50 \mu \mathrm{L} / \mathrm{min}$. These experiments included activation of the acoustic transducer ( $44 \mathrm{~mW}$ at $100 \%$ duty cycle) during binding and mixing steps, which resulted in a minimal improvement on binding and elution efficiency. Improving the DNA recovery using this cartridge would likely require additional optimization of power and flow settings.

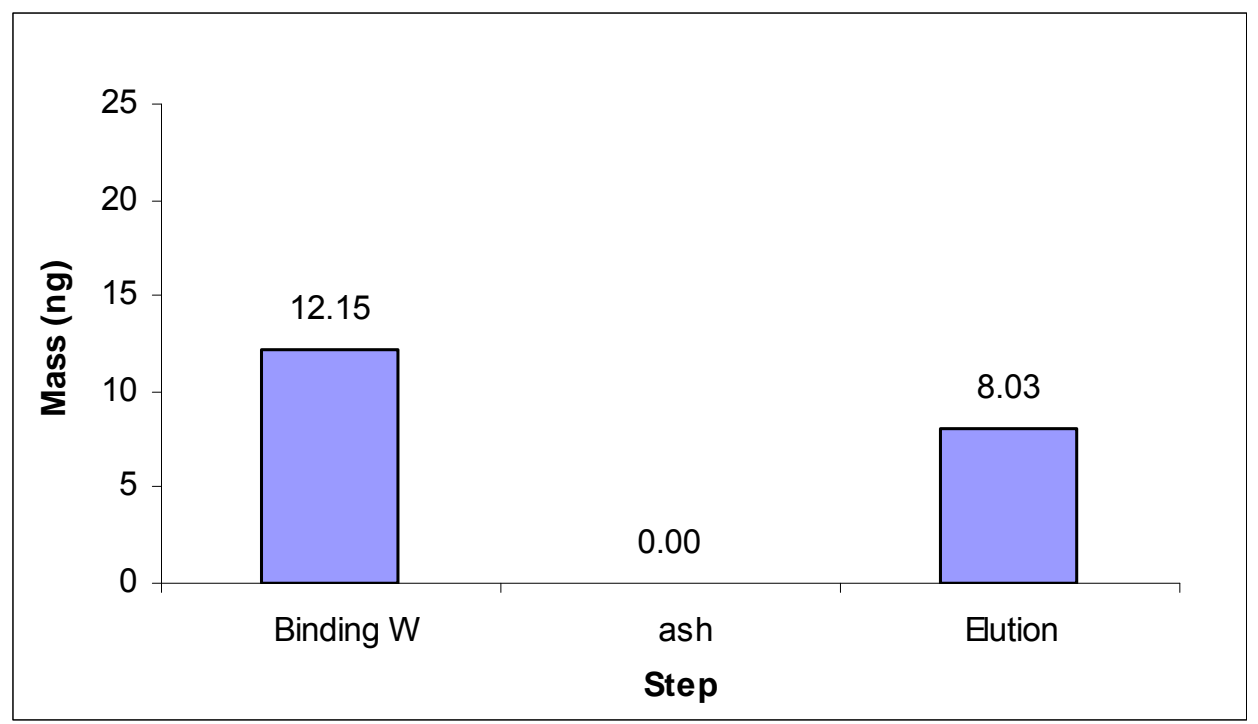

Figure 25 Mass of DNA extracted using ChargeSwitch ${ }^{\circledR}$ beads with microfluidic cartridge design 3 and a sample flow rate of $50 \mu \mathrm{L} / \mathrm{min}$.

For all three cartridge designs, a significant loss of DNA with the microfluidic channel was recorded. For the data presented in Figure 24 and Figure 25, 201 ng of DNA was loaded into the channel, of which, only $10-20 \%$ of which was recovered in the combined assay steps. A significant portion of the unrecovered DNA may have bound nonspecifically along the channel interior. Given that the cartridges were fabricated in plastic, the intrinsic surface charge may have bound DNA and caused additional sample loss. One method to mediate this effect is to passivate the microchannel to reduce the surface charge, thereby decreasing DNA binding. These cartridge designs may also be fabricated in glass using a laminate process similar to one employed here, with the exception of bonding layers using a thermal process. Glass fabricated microchannels would also permit higher pressure to be used, dramatically reducing the processing time.

\section{Nafion Coated Electrodes}

The third method investigated for nucleic acid extraction used an electric field to trap nucleic acid to a Nafion passivated gold electrode. This approach was based on preliminary results that showed passivation with Nafion maintained nucleic acid integrity and prevented electrode degradation. It was suspected that Nafion films (perfluorinated resin) preserve biomolecule integrity by preventing them from reaching the highly reactive electrode surface. The pores in the Nafion film were sufficiently small to prevent DNA migration while allowing ion mobility of cations to the electrode 
surface. The estimated thickness of the Nafion film was $12 \mu \mathrm{m}$. Using the assembled lysing/Nafion cartridges shown in Figure 17b, we measured the amount of DNA during the bound, wash and elution steps for two applied voltages. The total injected DNA was $90 \mathrm{ng}$ which gave in which $54.77 \mathrm{ng}$ bound at a voltage of $5.92 \mathrm{~V}$. This was later determined to exceed the binding capacity of the Nafion system. Maintaining the DC bias voltage until the elution step was crucial to prevent DNA loss during the wash step. For $90 \mathrm{ng}$ of injected DNA, we determined an extraction efficiency of $40.3 \%$, though additional DNA was lost due to limited binding capacity. The extraction efficiency increased to $66.3 \%$ when based on the DNA that actually bound and eluted from the Nafion electrodes.

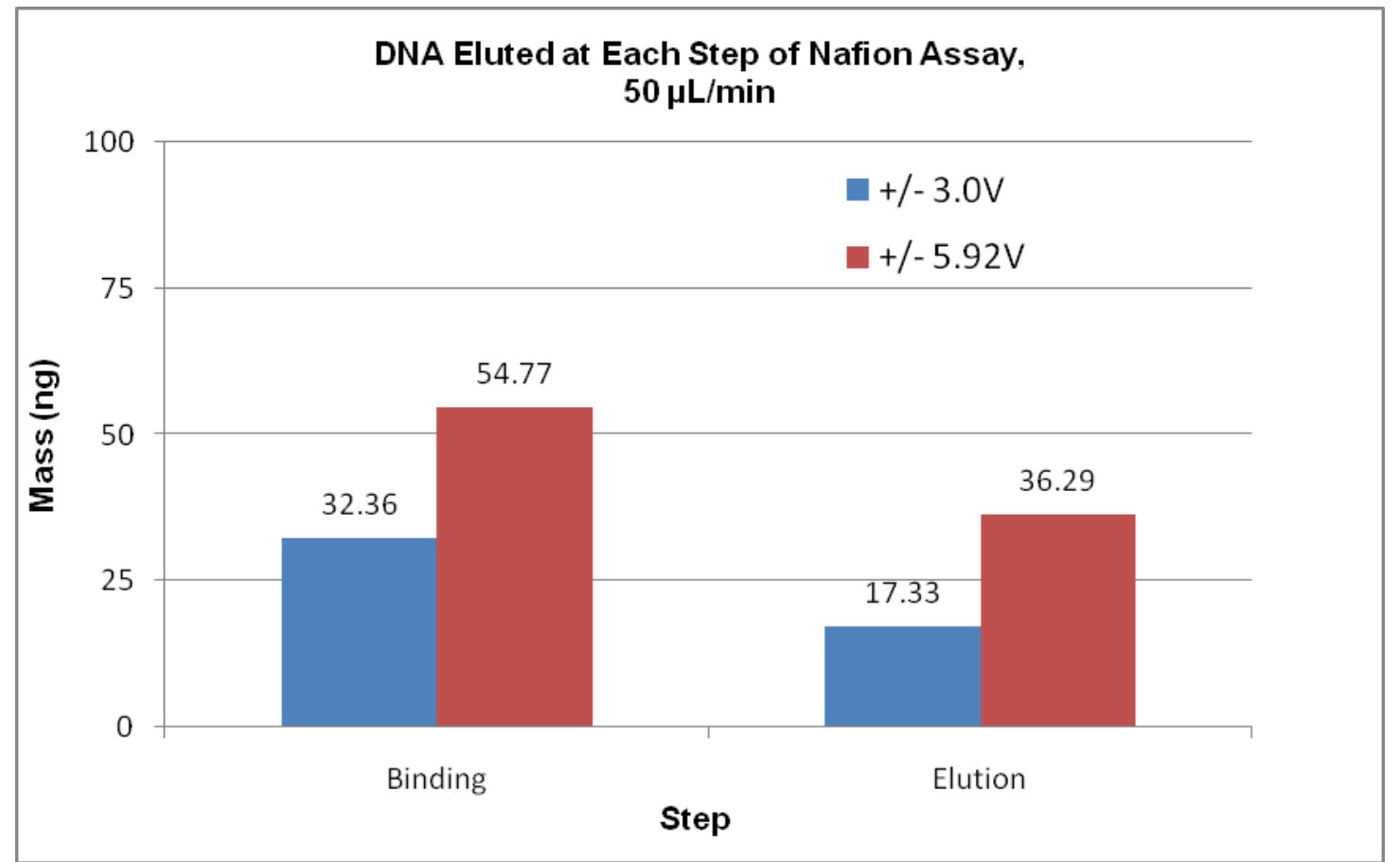

Figure 26 DNA extraction from Nafion coated electrodes. Increasing the applied voltage dramatically improved DNA binding. The injected amount of DNA was $90 \mathrm{ng}$.

\section{G. New DNA Detection Assay}

A key component of this LDRD is the sensitive detection of the DNA obtained through acoustic lysing techniques. We therefore focused our efforts upon developing alternate techniques and assays for the sensitive detection of DNA. Specifically, our objectives were: 1) Identify cyanine chromophores from our molecular library that would be a sensitive probe for nucleic acids; 2) Correlate the form-function aspects of cyanine chemistry to self-assembly on nucleic acid scaffolds; 3 ) Compare the detection limits of single-stranded viral DNA, double-stranded viral DNA, double-stranded genomic DNA, and RNA, using self-assembly; 4) Optimize detection conditions for nucleic acids based upon supramolecular self-assembly including the stability of the Nucleic acids-Cyanine complex; and 5) Demonstrate supramolecular self-assembly processes were involved in the aggregation of the cyanine upon nucleic acid scaffolds. Results from these investigations are described below. 


\section{Cyanine chemistry and spectral characterization}

We investigated the potential for J-aggregate formation of the various cyanines using nucleic acid as a template. The molecular structures and properties of four cyanines are shown in Fig. 1 . Among these, the cyanines designated $\mathbf{1}$ and $\mathbf{4}$ were capable of forming fluorescent J-aggregates and we therefore focused on these chromophores. Since we had access to $\mathbf{2}$ and $\mathbf{4}$, we tested these as well. Until now, there is no clear understanding of the relationship between cyanine chemistry and Jaggregation potential. By testing the four cyanines with subtle differences in their structures, three out of four had the electron withdrawing chlorine substituent (Figure 27), we hoped to better understand such relationships (vide infra).

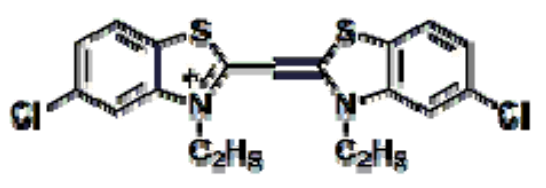

Mal. W4:; 499.39

1

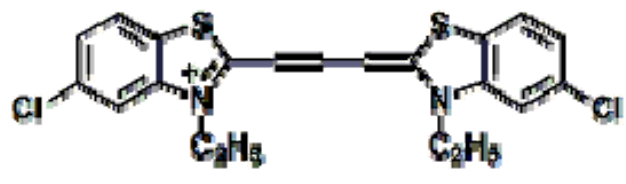

Mal, 4.4 tri 434,42

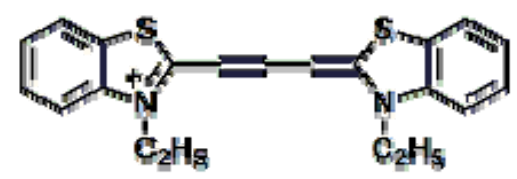

Mal, WH.; 365.53

2

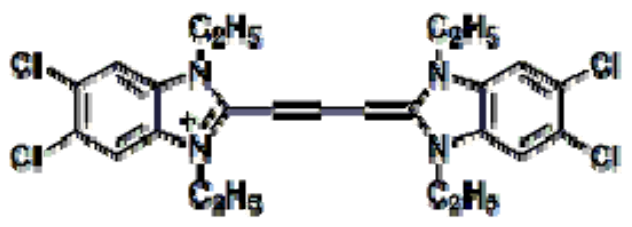

Mal, 44t: 32532

2

Figure 27 Molecular structures of the cyanines.

\section{Evidence for supramolecular self-assembly of cyanine 1 on nucleic acid scaffolds}

Cyanine dyes self-assemble upon DNA scaffolds through electrostatic and hydrophobic interactions. Consequently, reagents that promote or disrupt these interactions will influence the magnitude and extent of cyanine self-assembly upon DNA scaffold. It is clear from Figure 28 , that $20 \%(\mathrm{v} / \mathrm{v})$ methanol is both necessary and sufficient for maximal fluorescence from $\mathbf{1} \mathrm{J}$-aggregate upon four different types of nucleic acid scaffolds including RNA. The increase in fluorescence between 5 to $20 \%$ methanol might be due to increased solubility of the cyanine in the solvent, thus enhancing J-aggregate formation. On the other hand, higher concentrations of methanol inhibited the selfassembly by disrupting the hydrophobic interactions between the chromophore and the scaffold. This manifested in the biphasic curve shown in Figure 28. The concentration of methanol to effect $50 \%$ reduction in fluorescence intensity $\left(I C_{50}\right)$ was approximately the same for all four nucleic acid scaffolds, being $30-35 \%(\mathrm{v} / \mathrm{v})$. Methanol effects seen in Figure 28 are one piece of evidence in support of the supramolecular self-assembly of cyanine on nucleic scaffolds. 


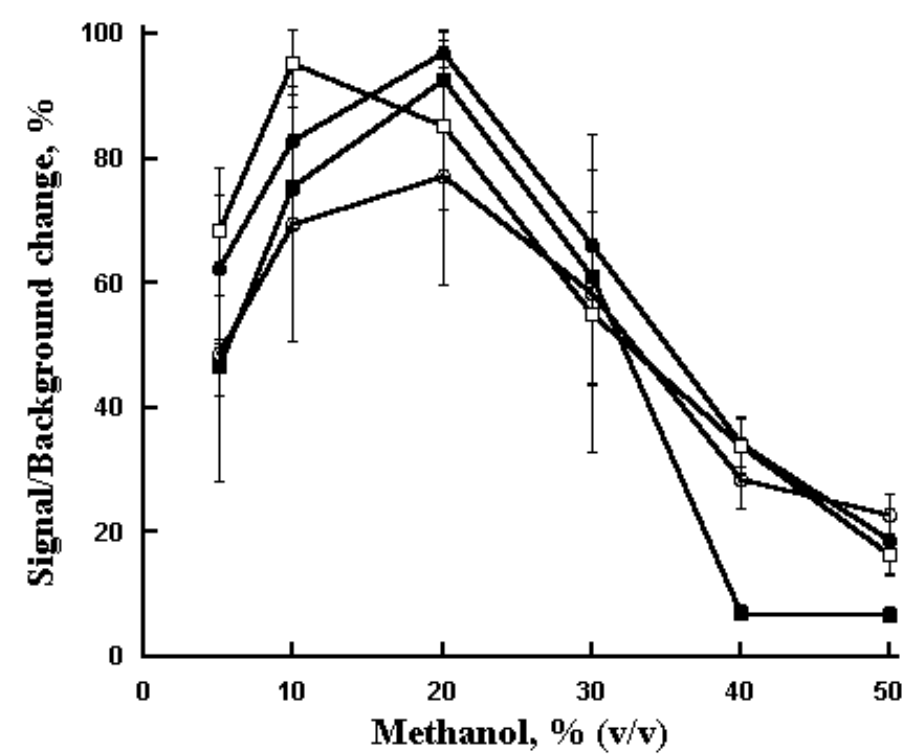

Figure 28 Effects of methanol concentration on the self-assembly of cyanine 1 . The tracing with open circles is \$X174 DNA, closed circles $\lambda$ DNA, open squares Escherichia coli genomic DNA and closed squares Saccharomyces cerevisiae RNA.

Another piece of evidence supporting supramolecular self-assembly of cyanine is the disruption by ionic strength. With the exception of RNA, salt uniformly inhibited selfassembly and decreased the fluorescence intensity (Figure 29). Unlike methanol profiles (Figure 28), the strength of $\mathrm{NaCl}$ inhibition was different with different scaffolds (Figure 29). Interaction between 1 and $\lambda$ DNA was most sensitive to ionic strength, whereas interactions of 1 with the other three nucleic acid scaffolds were more resistant to salt (Figure 29). The $\mathrm{NaCl}$ concentrations for $50 \%$ reduction in fluorescence intensity $\left(\mathrm{IC}_{50}\right)$ with the nucleic acid scaffolds were: $\lambda$ DNA $=10 \mathrm{mM} ; \phi X 174$ DNA and genomic DNA = $100 \mathrm{mM}$; and RNA = $75 \mathrm{mM}$. The dose-response profile of $\phi X 174$ DNA (double stranded, circular DNA) and E. coli genomic DNA (linear, double stranded DNA) was nearly identical (Figure 29). Salt effects could be rank-ordered as follows (least-to-most susceptible): $\phi X 174$ DNA = Genomic DNA > RNA > $\lambda$ DNA. The effect upon RNA was a departure from the rest of the nucleic acids, since it required $\sim 25 \mathrm{mM} \mathrm{NaCl}$ for maximum fluorescence, declining thereafter. On the other hand, there was no stimulatory effect $\mathrm{NaCl}$ upon the aggregation of 1 with the remaining nucleic acid scaffolds (Figure 29). 


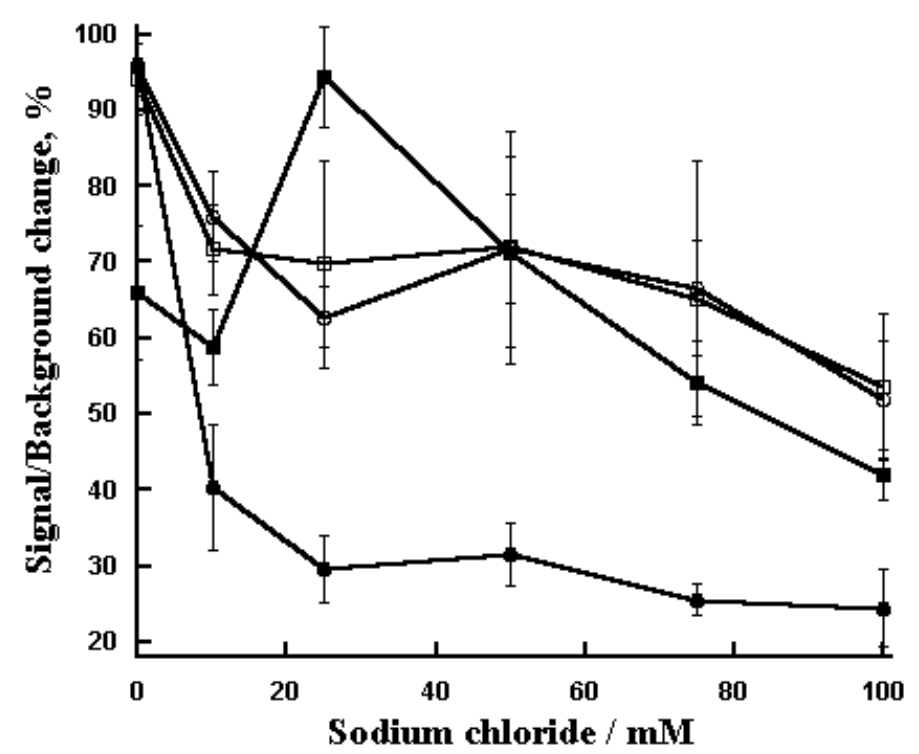

Figure 29 Effects of salt concentration on the self-assembly of cyanine 1. The tracing with open circles is $\phi X 174$ DNA, closed circles $\lambda$ DNA, open squares Escherichia coli genomic DNA and closed squares Saccharomyces cerevisiae RNA.

The salt permits neutralization of the cationic charge density of the aggregate and allows electrostatic screening between the anionic nucleic acids and the cationic dye. The salt and methanol sensitivities of $\lambda$ DNA strongly suggested a self-assembly mechanism based upon electrostatic (coulombic) and hydrophobic forces. With the remaining nucleic acid scaffolds, both self-assembly might be operative. Considering the effects of methanol and $\mathrm{NaCl}$ together, we speculate that non-covalent, reversible, supramolecular self-assembly is the dominant mechanism between $\mathbf{1}$ and the various nucleic acids.

The final evidence for supramolecular self-assembly of cyanine comes from temperature effects. Changes in temperature and ionic strength were crucial for inter-conversion between fluorescent J-aggregate and non-fluorescent or minimally fluorescent $\mathrm{H}$ aggregate. Depending upon the temperature, cyanine exists as monomer, $\mathrm{H}$-aggregate and $\mathrm{J}$-aggregate. Between the temperature range of 5 and $35^{\circ} \mathrm{C}$, cyanines formed $\mathrm{J}$ aggregates predominantly. Thermal stability of the J-aggregate is also dependent on the dye concentration. Since our experiments were conducted at $22^{\circ} \mathrm{C}$, within the window of J-aggregate-forming temperatures, and the high dye-to-DNA ratio (vide infra), we believe that the 1 was undergoing supramolecular self-assembly upon the nucleic acid scaffolds.

\section{Cyanine 1 optimization for detecting nucleic acids}

The optimum concentration for detecting and quantizing $\phi X 174$ virion DNA was 2.5-5 $\mu \mathrm{M}$ of 1 (Figure 30). Similar biphasic trends were observed during dose-response titrations with the other nucleic acids, where the optimum concentration of 1 was 5-10 $\mu \mathrm{M}$. As noted above, dye aggregation is influenced by several parameters, i.e., the type and concentration of the nucleic acid and the dye, ionic strength, $\mathrm{pH}$ and temperature. 


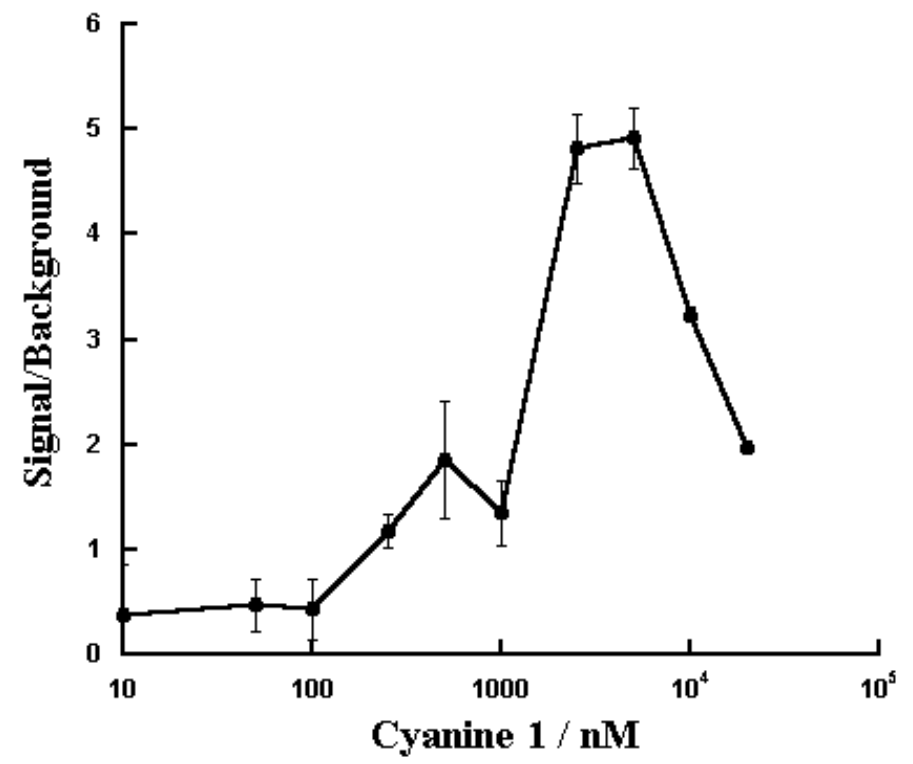

Figure 30 Optimization of cyanine 1 concentration for the detection and quantization of $\$$ X174 DNA. Increasing concentrations of 1 were mixed with 450 pM $\phi X 174$ virion DNA in a total volume of $100 \mu \mathrm{L}$ (45 fmol DNA) and fluorescence intensity was measured by exciting the samples at $425 \mathrm{~nm}$ and emission at $470 \mathrm{~nm}$.

\section{DNA quantization using cyanine 1}

We next constructed a calibration curve of $\phi X 174$ DNA with $5 \mu \mathrm{M}$ of 1 (Figure 31). We calculated limit of detection (LOD) of $3 \mathrm{fmol}$ DNA and a limit of quantitation (LOQ) of $9 \mathrm{fmol}$ (30 and $90 \mathrm{pM} ; 50$ and $150 \mathrm{ppb}$, respectively). Linearity of doseresponse and the overlap between data and curve fit were good (Figure 31). The sensitivity for single stranded DNA were comparable to those reported using double stranded DNA [17]. Our assay can be miniaturized to ultra-high-throughput screening (uHTS) with 1536-wells using the principles described by us previously [18]. The sensitivity for other nucleic acids ranged between 25 to $50 \mathrm{ng} / \mathrm{mL}$ (LOD) and 75 to 150 $\mathrm{ng} / \mathrm{mL}$ (LOQ), with the exception of RNA, where LOQ was $250 \mathrm{ng} / \mathrm{mL}$. Whereas 1 was equally sensitive toward single stranded, double stranded, linear and circular DNA, it was less sensitive for RNA. The reasons for this may relate to structural differences of the chromophore and/or the scaffold. The foregoing work has been published in Analytical Sciences [19], an international journal published by The Japan Society for Analytical Chemistry. 


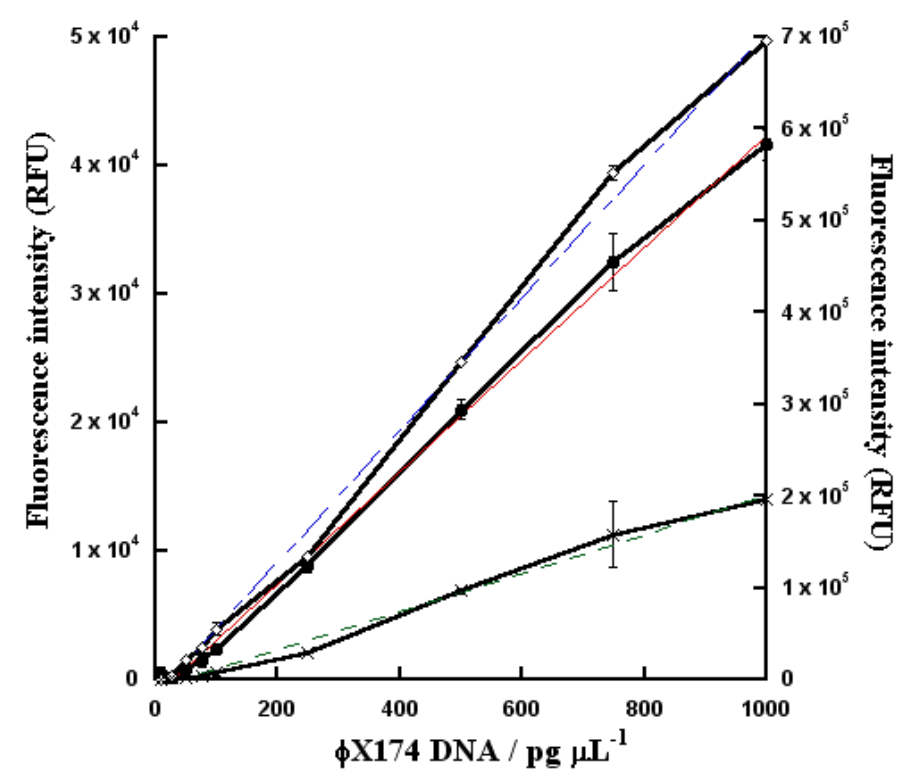

Figure 31 Quantization of $\phi X 174$ DNA using the supramolecular self-assembly of cyanine 1 . Increasing amounts of фX174 DNA were reacted with $5 \mu \mathrm{M}$ 1. The closed circles data were collected with $355 \mathrm{~nm}(\mathrm{ex})$ and $460 \mathrm{~nm}$ (em) filters (left, Y-1 axis). The open diamonds data were obtained with $400 \mathrm{~nm}$ (ex) and $460 \mathrm{~nm}$ (em) filters (right, Y-2 axis). The cross symbols data were obtained using the monochromator-based instrument with $426 \mathrm{~nm}$ (ex) and 470 $\mathrm{nm}(\mathrm{em})$ (left, Y-1 axis). Linearity $\left(r^{2}\right)$ of the dose-response curves were: open diamonds, 0.99192; closed circles, 0.99830 ; crosses, 0.99675 . 


\section{Conclusions}

In this work, we presented a novel nucleic acid extraction technology based on the efficient lysis of cellular samples using a BAW transducer array coupled to removable microchannels. A BAW transducer array was developed to efficiently and rapidly lyse samples for either subsequent use or further processing on the cartridge. The cartridges implemented three types of DNA extraction methods: sol-gel silica bead matrices, paramagnetic nucleic acid binding beads, and electric field extraction using Nafion films on gold electrodes. Several computational models were developed for optimization and design. Specifically, the thermal model showed the distribution within the microchannel versus flow rate. This thermal model also dramatically improved over existing reports where the effect was not even considered. We discovered that thermal effects can dramatically limit the success of any acoustic based lysing system, specifically where the protein content may be of interest. We demonstrate the successful lysing and DNA extraction from $E$. coli samples in plastic and glass based cartridges at levels sufficient for molecular beacon and PCR detection applications. Our results demonstrate the ability to lyse cells using a completely plastic based microchannel, which substantially simplifies fabrication complexity and cost compared to microchannels fabricated from both plastic and glass. Moreover, we have demonstrated supramolecular selfassembling cyanine as a useful alternative to the sensitive detection of various types of nucleic acids including DNA and RNA for microformat applications. We expect that this technology will have wide application for existing biosensor systems and offer excellent scalability for multi-analyte detection applications. 


\section{Appendix}

Table 3 Measured Acoustic Properties of Epotek Epoxies ( $f=5 \mathrm{MHz}$ )

\begin{tabular}{|c|c|c|c|c|c|}
\hline Epoxy & $\begin{array}{c}\text { Thickness } \\
(\mathbf{m m})\end{array}$ & $\begin{array}{c}\text { Density } \\
\left(\mathbf{k g} / \mathbf{m}^{\mathbf{3}}\right)\end{array}$ & $\begin{array}{c}\text { Longitudinal } \\
\text { velocity } \\
(\mathbf{m} / \mathbf{s})\end{array}$ & $\begin{array}{c}\text { Impedance } \\
\text { (MRays) }\end{array}$ & $\begin{array}{c}\text { Loss } \\
\text { (dB/cm) }\end{array}$ \\
\hline $\mathrm{E} 4110$ & 1.56 & 2905 & 4052 & 11.7 & 56.4 \\
\hline $\mathrm{H} 24$ & 1.35 & 2050 & 4804 & 9.8 & 63.7 \\
\hline $\mathrm{E} 4110-\mathrm{LV}$ & 1.31 & 2910 & 4094 & 11.9 & 53.4 \\
\hline $\mathrm{H} 20-\mathrm{E}$ & 1.31 & 2550 & 4094 & 10.4 & 75.8 \\
\hline 301 & 0.52 & 1094 & 5778 & 6.3 & 25.9 \\
\hline
\end{tabular}

\section{Developed Computer Codes}

1. Loss tangent to attenuation conversions.

2. Effective permittivity computation for arbitrary piezoelectric materials and orientations. Analysis determines the allowed wave types and their velocities.

3. Green's function tensor computation for arbitrary piezoelectric materials and orientations. Analysis determines the allowed wave types, velocities and the relationship between the displacements and potential and induced stresses and electric displacement.

4. 1D transmission line analysis of BAW devices.

5. 2D and 3D FEM of BAW devices.

6. 3D FEM thermal-fluid transport analysis of microfluidic devices. 


\section{References}

[1] P. Belgrader, D. Hansford, G. T. A. Kovacs, K. Venkateswaran, J. R. Mariella, F. Milianovich, S. Nasarabadi, M. Okuzumi, F. Pourahmadi, and M. A. Northrup, "A minisonicator to rapidly disrupt bacterial spores for DNA analysis," Anal. Chem., vol. 71, pp. 4232-4236, 1999.

[2] G. Zhang, K. Hashimoto, and M. Yamaguchi, "Liquid streaming by high-frequency ultrasonic waves," Jpn. J. Appl. Phys., vol. 35, pp. 3248-3250, 1996.

[3] D. P. Chandler, J. Brown, C. J. Bruckner-Lea, L. Olson, G. J. Posakony, J. R. Stults, N. B. Valentine, and L. J. Bond, "Continous spore disruption using radially focused, high-frequency ultrasound," Anal. Chem., vol. 73, pp. 3784-3789, 2001.

[4] T. C. Marentis, B. Kusler, G. G. Yaralioglu, S. Liu, E. O. Hæggstöm, and B. T. KuriYakub, "Microfluidic sonicator for real-time disruption of eukaryotic cells and bacterial spores for DNA analysis," Ultrasound in Med. \& Biol., vol. 31, pp. 12651277, 2005.

[5] H. Jagannathan, G. G. Yaralioglu, A. S. Ergun, F. L. Degertekin, and B. T. KhuriYakub, "Micro-fluidic channels with integrated ultrasonic transducers," IEEE Ultrason. Symp., pp. 859-862, 2001.

[6] N. C. Cady, S. Stelick, and C. A. Batt, "Nucleic acid purification using microfabricated silicon structures," Biosens. Bioelectr., vol. 19, pp. 59-66, 2003.

[7] M. Moré, J. B. Herrick, M. C. Silva, W. C. Ghiorse, and E. L. Madsen, "Quantitative cell lysis of idigenous microorganisms and rapid extraction of microbial DNA from sediment," Appl. Environ. Microbiol., vol. 60, pp. 1572-1580, 1994.

[8] R. Boom, C. J. A. Sol, M. M. M. Salimans, C. L. Jansen, P. M. E. W.-v. Dillen, and J. v. d. Noordaa, "Rapid and simple method for purificaition of nucleic acids," $J$. Clin. Microbiol., vol. 28, pp. 495-503, 1990.

[9] K. Wolfe, M. C. Breadmore, J. P. Ferrance, M. E. Power, J. F. Conroy, P. M. Norris, and J. P. Landers, "Toward a microchip-based solid phase extraction method for isolation of nucleic acids," Eletrophoresis, vol. 23, pp. 727-733, 2002.

[10] M. Lee, J.-N. Lee, J. Kim, and J.-H. Kim, "Reversible capture of genomic DNA by a Nafion-coated electrode," Analy. Biochem., vol. 380, pp. 335-337, 2008.

[11] H. Allik and T. J. R. Hughes, "Finite element method for piezoelectric vibration," Int. J. Numer. Methods Eng., vol. 2, pp. 151-157, 1970.

[12] L. P. Gor'kov, "On the forces acting on a small particle in an acoustical field in an ideal fluid," Soviet Physics-Doklady, vol. 6, pp. 773-775, 1962.

[13] J.-L. Dion, E. Cornieles, F. Galindo, and K. Agbossou, "Exact one-dimensional computation of ultrasonic transducers with several piezoelectric elements and passive layers using the transmission line analogy," IEEE Trans. Ultrason. Ferrelect. Freq. Contr., vol. 44, pp. 1120-1131, 1997.

[14] P. E. Bloomfield, W.-J. Lo, and P. A. Lewin, "Experimental study of the acoustical properties of polymers utilized to construct PVDF ultrasonic transducers and the acousto-electric properties of PVDF and P(VDF/TrFE) films," IEEE Trans. Ultrason. Ferrelect. Freq. Contr., vol. 47, pp. 1397-1405, 2000. 
[15] A. A. Miles and S. S. Misra, "The bactericidal power of the blood," Journal of Hygeine, vol. 38, pp. 732-748, 1938.

[16] V. R. Esche, "Untersuchung der schwingungskavitation in flüssigkeiten," Acoustica 2, pp. AB 208-218, 1952.

[17] K. Renegarajan, S. M. Cristol, M. Mehta, and J. M. Nickerson, "Intercalating Gold Nanoparticles as Universal Labels for DNA Detection," Mol. Vision, vol. 8, p. 416, 2002.

[18] D. W. Branch, J. Sigman, C. D. James, P. G. Clem, and K. E. Achyuthan, "On-Chip Biological Sample Preparation System using Acoustic Lysis," in Biomed. Eng Soc., Los Angeles, CA, 2007.

[19] K. E. Achyuthan, J. L. McClain, Z. Zhou, D. G. Whitten, and D. W. Branch, "Spectroscopic Analyses of the Noncovalent Self-Assembly of Cyanines upon Various Nucleic Acid Scaffolds," Analyt. Sci., vol. 25, p. 469, 2009.

\title{
VIII. Distribution List
}

$\begin{array}{ll}4 & \text { MS } 1425 \\ 1 & \text { MS } 1425 \\ 2 & \text { MS } 1425 \\ 1 & \text { MS } 0899\end{array}$

\author{
Darren W. Branch \\ Erika J. Cooley \\ Steve Casalnuovo \\ Technical Library (electronic files), 9536
}


